Florida International University

FIU Digital Commons

FIU Electronic Theses and Dissertations

University Graduate School

7-7-2008

\title{
Synthesis and investigation of highly conductive Cu-Cr-MWCNT composites
}

Puneet K. Singh Gill

Florida International University

Follow this and additional works at: https://digitalcommons.fiu.edu/etd

Part of the Materials Science and Engineering Commons

\section{Recommended Citation}

Gill, Puneet K. Singh, "Synthesis and investigation of highly conductive Cu-Cr-MWCNT composites" (2008). FIU Electronic Theses and Dissertations. 3938.

https://digitalcommons.fiu.edu/etd/3938

This work is brought to you for free and open access by the University Graduate School at FIU Digital Commons. It has been accepted for inclusion in FIU Electronic Theses and Dissertations by an authorized administrator of FIU Digital Commons. For more information, please contact dcc@fiu.edu. 


\title{
FLORIDA INTERNATIONAL UNIVERSITY
}

\author{
Miami, Florida
}

\section{SYNTHESIS AND INVESTIGATION OF HIGHLY CONDUCTIVE Cu-Cr-MWCNT COMPOSITES}

\author{
A thesis submitted in partial fulfillment of the \\ requirements for the degree of \\ MASTER OF SCIENCE \\ in \\ MATERIALS SCIENCE AND ENGINEERING \\ by \\ Puneet K. Singh Gill
}


To: Interim Dean Amir Mirmiran

College of Engineering and Computing

This thesis, written by Puneet K. Singh Gill, and entitled Synthesis and Investigation of Highly Conductive $\mathrm{Cu}-\mathrm{Cr}-\mathrm{MWCNT}$ Composites, having been approved in respect to style and intellectual content, is referred to you for judgment.

We have read this thesis and recommend that it be approved.

Arvind Agarwal

Wonbong Choi

Norman Munroe, Major Professor

Date of Defense: July 7, 2008

The thesis of Puneet K. Singh Gill is approved.

Interim Dean Amir Mirmiran College of Engineering and Computing

Dean George Walker University Graduate School

Florida International University, 2008 


\section{ACKNOWLEDGMENTS}

I take this opportunity to express my sincere gratitude towards Dr. Norman Munroe (Faculty Advisor) for his support, motivation and valuable discussions during this entire research work. His continuous guidance helped me immensely in the research.

My sincere thanks go to Dr. Arvind Agarwal and Dr. Wonbong Choi for serving on my thesis committee and for their valuable suggestions. I also wish to acknowledge the Applied Research Center, Mechanical Materials Engineering Department, Advanced Materials Engineering Research Institute and Future Aerospace Science and Technology Center for Space Cryoelectronics for laboratory support.

Special words of thanks go to Sushma Amruthaluri who provided extensive assistance in the experimental work. I thank my friends, Kantesh Balani, Waseem Haider, Chandan Pulletikurthi, Amardeep Mann and Srinivasa Bakshi for all their help and support.

I would also like to thank my lovely parents and brother who supported me with their love, in every moment, during ups and downs of this research. Above all, I would like to thank my fiance Zinki, without her moral support and love I would not be able to complete my thesis work successfully. 


\section{ABSTRACT OF THE THESIS \\ SYNTHESIS AND INVESTIGATION OF HIGHLY CONDUCTIVE \\ Cu-Cr-MWCNT COMPOSITES}

by

Puneet K. Singh Gill

Florida International University, 2008

Miami, Florida

Professor Norman Munroe, Major Professor

There is great demand for contact materials with superior electrical and thermal conductivities and mechanical strength, for use in Vacuum Circuit Breakers (VCB). Copper $(\mathrm{Cu})$ and chromium $(\mathrm{Cr})$ alloy, developed in the 1970's [1] has been the most common contact material in VCBs. Although $\mathrm{Cu}-\mathrm{Cr}$ alloys possess good conductivity and mechanical properties, $50 \%$ of $\mathrm{Cr}$ (a strategic metal) in the alloy is resource prohibitive. A reduction in $\mathrm{Cr}$ usage without compromising desired properties is desirable.

Previous researchers focused on developing $\mathrm{Cu}-\mathrm{Cr}$ alloys with fine and uniform microstructure to enhance their physical properties. This investigation focused on the development of a $\mathrm{Cu}-\mathrm{Cr}-\mathrm{MWCNT}$ (Multi walled Carbon Nanotubes) composite with enhanced properties as compared with currently used materials. The electrical conductivity of the composite increased up to 18 times that of $\mathrm{Cu}$ and there was also an increase in the Vicker's hardness. 


\section{TABLE OF CONTENTS}

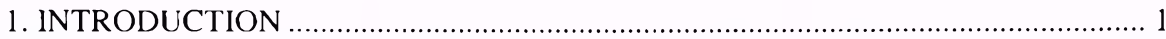

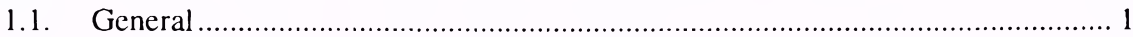

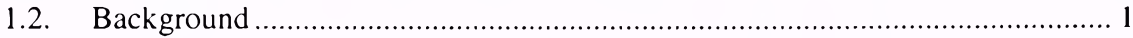

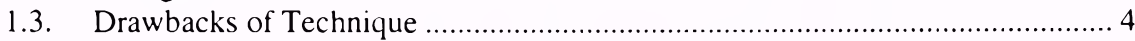

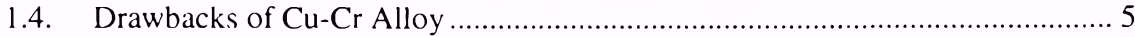

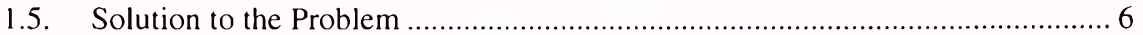

1.5.1. Effect of Content of CNT in Cu-CNT Composite ............................................ 6

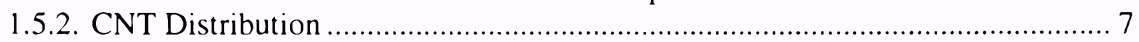

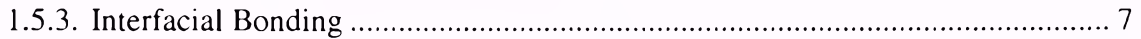

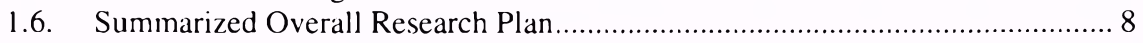

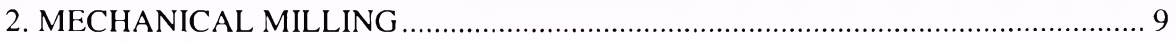

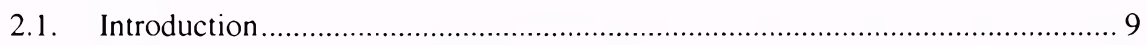

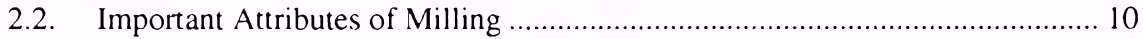

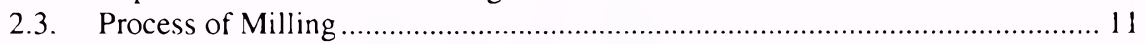

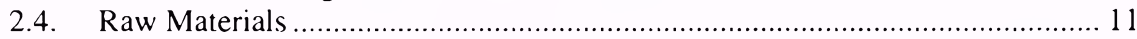

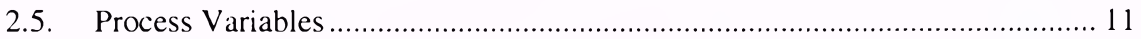

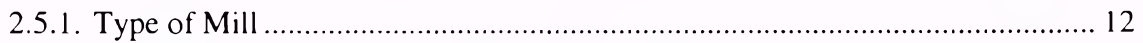

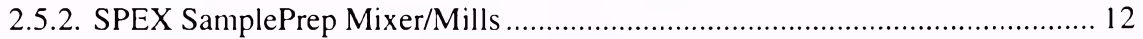

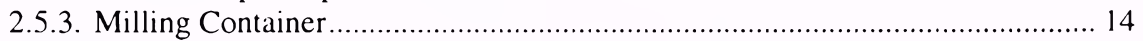

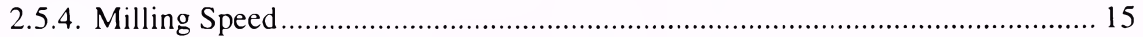

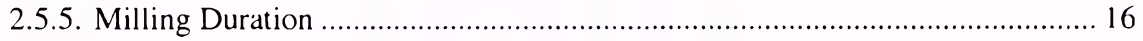

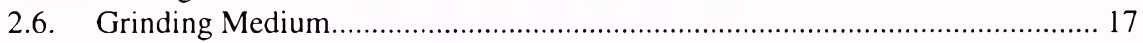

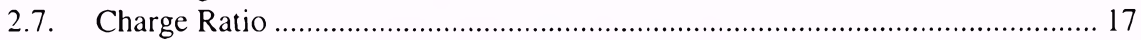

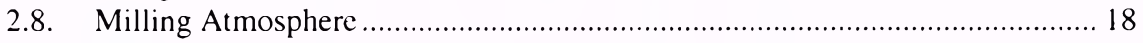

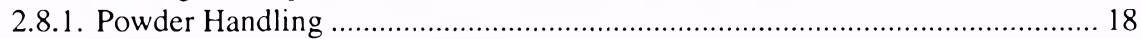

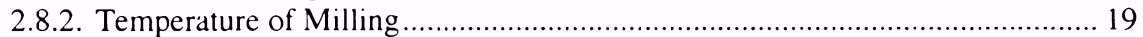

2.9. Milling Parameters Considered in this Research ......................................... 19

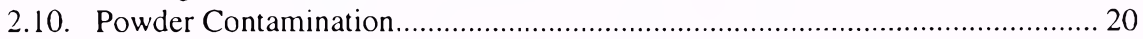

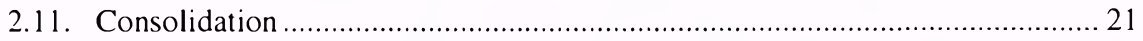

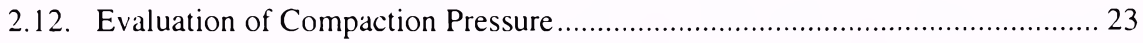

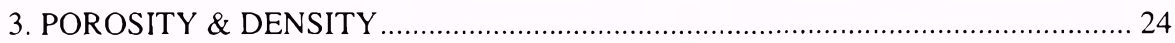

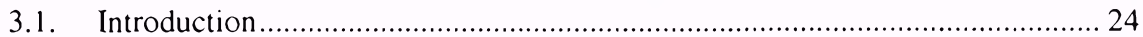

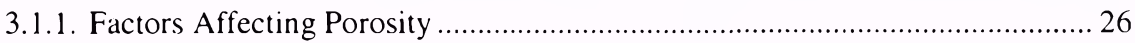

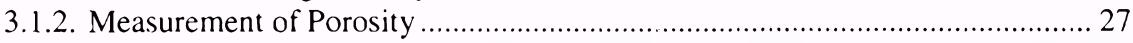

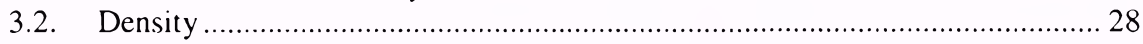

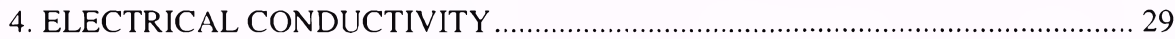




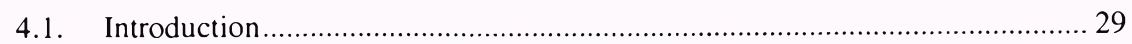

4.2. Effect of Temperature on Electrical Conductivity ........................................... 29

4.3. Effect of Porosity on Electrical Conductivity ................................................. 30

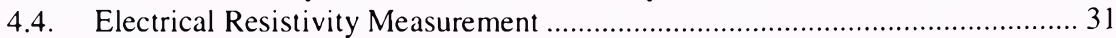

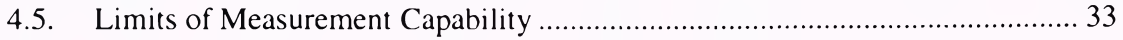

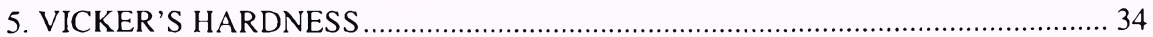

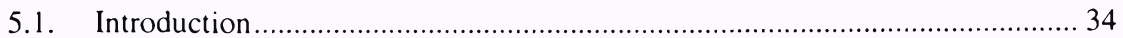

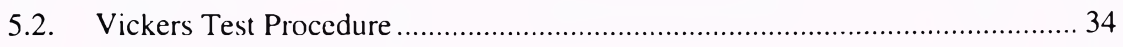

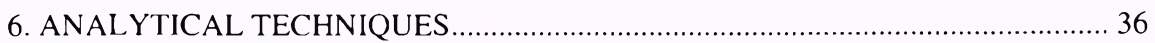

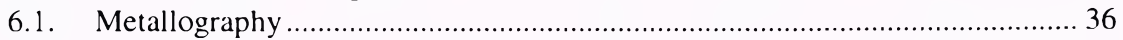

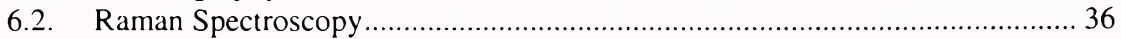

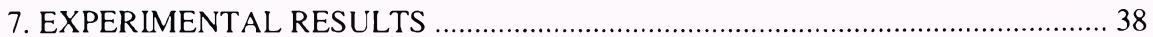

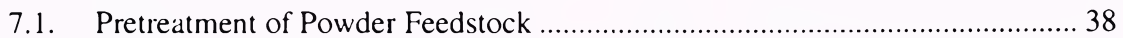

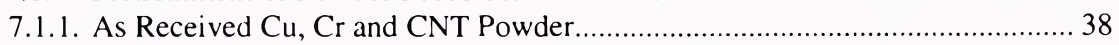

7.2. Selection of Appropriate Material and Milling Time ...................................... 39

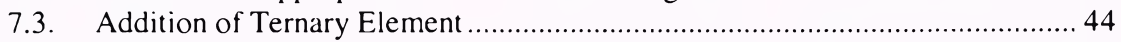

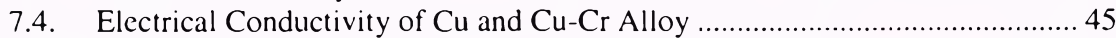

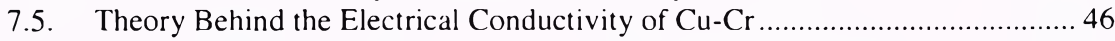

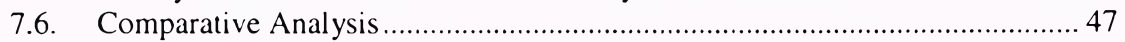

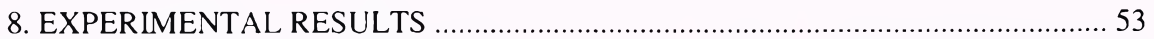

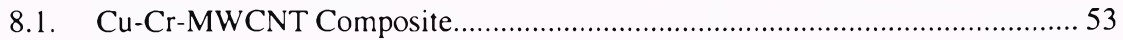

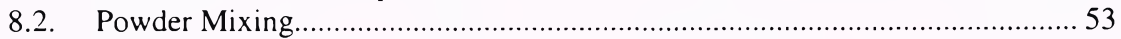

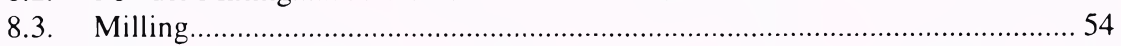

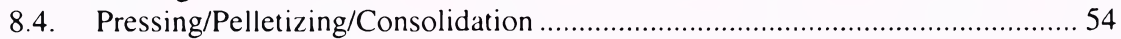

8.5. SEM/EDS Analysis of the Samples Before Sintering ……............................ 55

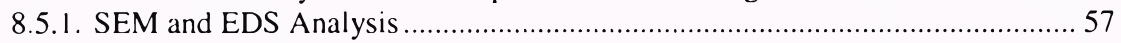

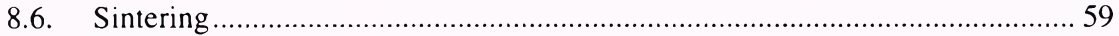

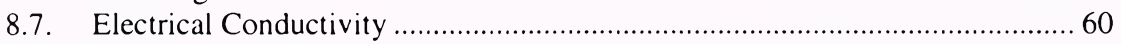

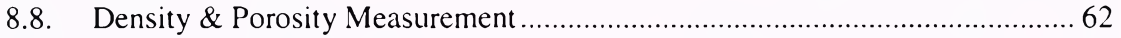

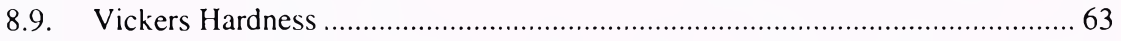

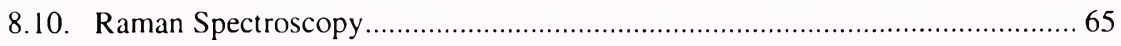

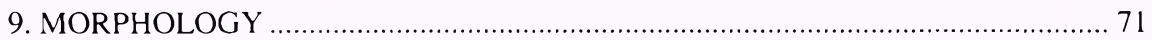

9.1. Scanning Electron Microscopy Analysis .................................................... 71

9.2. SEM/EDS and Mapping Analyses of samples............................................... 73

9.3. TEM Analysis of $\mathrm{Cu}-\mathrm{Cr} 10 \mathrm{Vol}$. \% MWCNT Composite ............................... 97

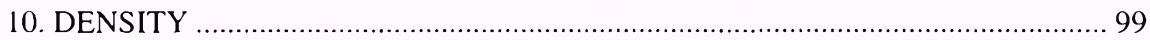

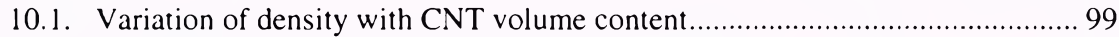

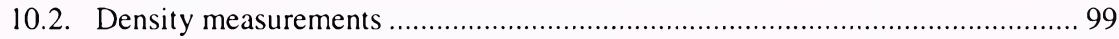

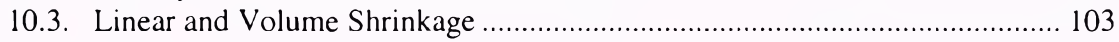




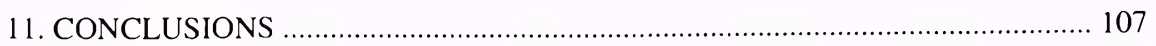

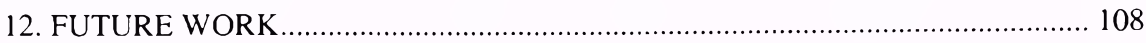

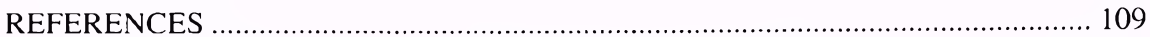

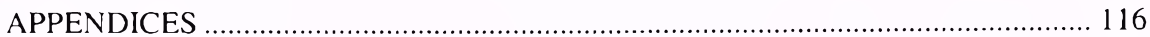




\section{LIST OF TABLES}

TABLE

PAGE

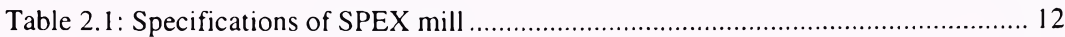

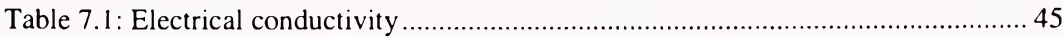

Table 8.1: Compositions of $\mathrm{Cu}-\mathrm{Cr}$ alloy and composites.............................................. 53

Table 8.2: Electrical conductivity at $290 \mathrm{~K}$ of composites, sintered at $750{ }^{\circ} \mathrm{C}$.................61 61

Table 8.3: Electrical conductivity at $290 \mathrm{~K}$ of composites, sintered at $900{ }^{\circ} \mathrm{C}$................. 61

Table 8.4: Variation of porosity with MWCNT volume percentage at three sintering

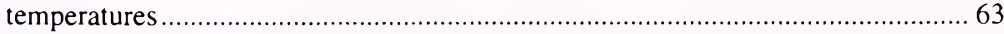

Table 8.5: Vicker's hardness of Cu-Cr MWCNT composites .......................................... 64

Table 8.6: Comparison of the spectral parameters in the Raman spectra before

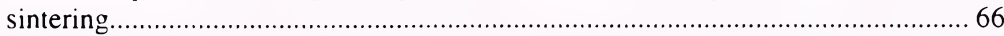

Table 8.7: Comparison of the spectral parameters in the Raman spectra of samples sintered at $750{ }^{\circ} \mathrm{C}$.

Table 8.8: Comparison of the spectral parameters in the Raman spectra of composites

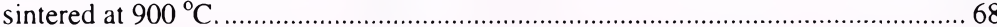

Table 8.9: Comparison of the spectral parameters in the Raman spectra of composites sintered at $1050^{\circ} \mathrm{C}$.

Table 10.1: Density measure for pure Cu sample...................................................... 100

Table 10.2: Density of Green pellet at three different sintering temperatures............... 100

Table 10.3: Volume shrinkage with variation of composition and sintering temperatures 104

Table 10.4: Linear shrinkage variations at different sintering temperatures. 105 


\section{LIST OF FIGURES}

FIGURE

PAGE

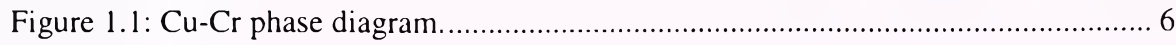

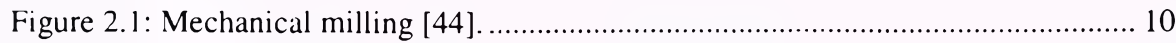

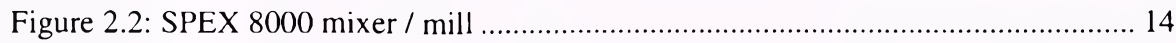

Figure 2.3: Hardened steel vial with stainless steel ball set........................................ 15

Figure 2.4: Refinement of particle and grain sizes with milling time and BPR [49]. ..... 16

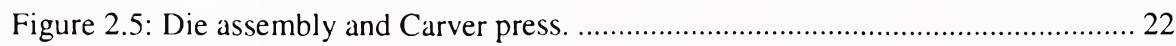

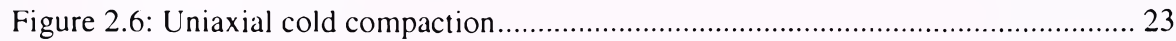

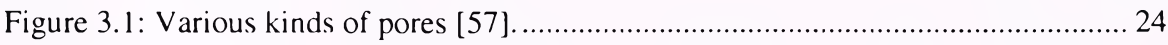

Figure 3.2: Variation of composite relative density with CNT volume content [76]...... 28

Figure 4.1: Four probe HP 4263A LCR meter. .............................................................. 31

Figure 4.2: Schematic representation of the Four-point probe ..................................... 32

Figure 5.1: Schematic of Vickers hardness test [59] ................................................... 35

Figure 7.1: As-received Cu powder (left) and $\mathrm{Cr}$ powder (right) particles. .................... 38

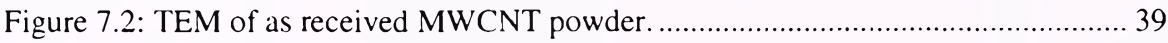

Figure 7.3: SEM of Cr particles after 15 minutes of ball milling.................................. 40

Figure 7.4: SEM of Cr particles after 45 minutes of ball milling................................... 40

Figure 7.5: SEM of Cr particles after 60 minutes of ball milling ................................. 41

Figure 7.6: SEM of $\mathrm{Cr}$ particles after 120 minutes of ball milling.............................. 41

Figure 7.7: SEM of Cr particles after 180 minutes of ball milling ................................. 41

Figure 7.8: SEM of Cr particles after 240 minutes of ball milling.............................. 41

Figure 7.9: Plot of average particle size of $\mathrm{Cr}$ (micron) versus milling time (minutes)... 42

Figure 7.10: $\mathrm{Cu} \mathrm{Cr} 15$ wt. \% after 15 minutes of ball milling........................................ 42 
Figure 7.11: $\mathrm{Cu} \mathrm{Cr15}$ wt. \% after 30 minutes of ball milling.................................. 42

Figure 7.12: Cu Cr15wt. \% after 45 minutes of ball milling. .................................. 43

Figure 7.13: Cu Cr15 wt. \% after 60 minutes of ball milling................................. 43

Figure 7.14: Cu Cr15wt. \% after 120 minutes of ball milling..................................... 43

Figure 7.15: $\mathrm{Cu} \mathrm{Cr15wt.} \mathrm{\%} \mathrm{after} 150$ minutes of ball milling.................................. 43

Figure 7.16: $\mathrm{Cu} \mathrm{Cr15wt.} \mathrm{\%} \mathrm{after} 180$ minutes of ball milling................................. 43

Figure 7.17: Cu Cr15wt. \% after 240 minutes of ball milling.................................. 43

Figure 7.18: Plot of average particle size of $\mathrm{Cu}-\mathrm{Cr} 15$ wt.\% composite versus

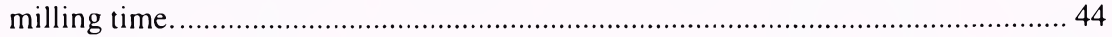

Figure 7.19: Electrical conductivity of $\mathrm{Cu}$ and $\mathrm{Cu}-\mathrm{Cr}$ alloys. ................................ 45

Figure 7.20: Conductivity versus temperature of $\mathrm{Cu}$ compact and $\mathrm{Cu}$ plate............... 46

Figure 7.21: SEM analyses for pure Cu plate (i.e. C26000)................................... 47

Figure 7.22: EDS analyses for pure Cu plate (i.e. C26000) ..................................... 48

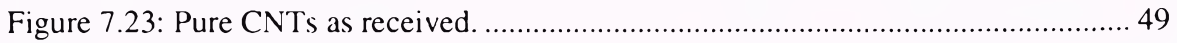

Figure 7.24: EDS analyses of pure CNTs as received....................................... 49

Figure 7.25: CNTs sintered at $750{ }^{\circ} \mathrm{C}$ in an inert atmosphere. ................................ 50

Figure 7.26: EDS analysis of pure CNTs powder sintered at $750{ }^{\circ} \mathrm{C}$ under an inert

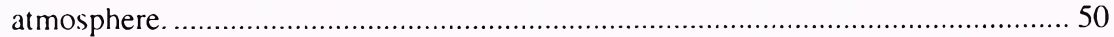

Figure 7.27: SEM image of pure CNTs powder sintered at $900{ }^{\circ} \mathrm{C}$ under an inert atmosphere.

Figure 7.28: EDS analysis of pure CNTs powder sintered at $900{ }^{\circ} \mathrm{C}$ under an inert atmosphere.

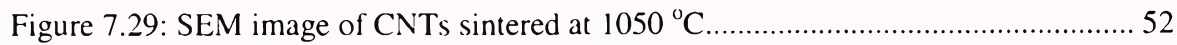

Figure 7.30: EDS analysis of pure CNTs powder sintered at $1050{ }^{\circ} \mathrm{C}$ under an inert atmosphere.

Figure 8.1: Dependency of Compactness of CNTs reinforced Cu based composite on the content of CNTs [31]. 
Figure 8.2: SEM analyses for $\mathrm{Cu}-\mathrm{Cr}$ composite before sintering.

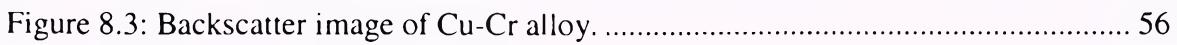

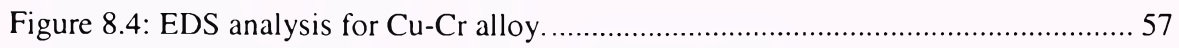

Figure 8.5: SEM image of $\mathrm{Cu}-\mathrm{Cr} 8 \mathrm{Vol}$. \% MWCNT composite..................................... 57

Figure 8.6: Backscatter image of $\mathrm{Cu}-\mathrm{Cr} 8 \mathrm{Vol}$. \% MWCNT composite......................... 58

Figure 8.7: EDS analysis of Cu-Cr 8 Vol. \% MWCNT composite.................................... 58

Figure 8.8: SEM image of $\mathrm{Cu}-\mathrm{Cr} 10 \mathrm{Vol}$ \% MWCNT ................................................. 59

Figure 8.9: SEM image of $\mathrm{Cu}-\mathrm{Cr} 12$ Vol. \% MWCNT .............................................. 59

Figure 8.10: Backscatter image of Cu-Cr 14 Vol. \% MWCNT ........................................ 59

Figure 8.11: Backscatter image of Cu-Cr 16 Vol. \% MWCNT......................................... 59

Figure 8.12: Lindberg/Blue tube furnace, with quartz tube flooded with argon. ............. 60

Figure 8.13: Plot of Electrical conductivity Vs Temperature for samples sintered at $750^{\circ} \mathrm{C}$

Figure 8.15: Variation of porosity with MWCNT volume percentage at three different

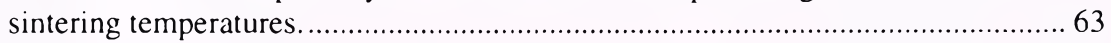

Figure 8.16: Dependency of Compactness of CNTs reinforced Cu based composite

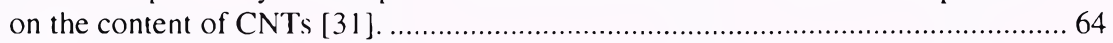

Figure 8.17: Variation of Vicker's hardness of composites with MWCNT content. ....... 65

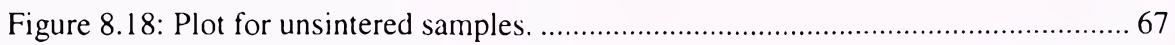

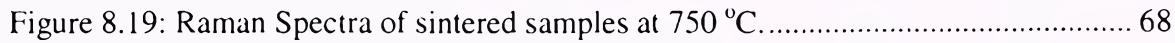

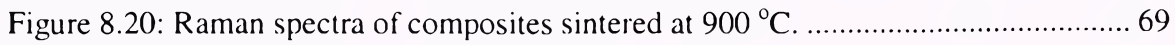

Figure 8.21: Raman spectra of composites sintered at $1050{ }^{\circ} \mathrm{C}$................................... 70

Figure 8.22: Variation of intensity ratio with MWCNT Vol. \% at three different sintering temperatures........................................................................................ 70

Figure 9.1: SEM image of the ball milled powder composite with $\mathrm{Cu}-\mathrm{Cr} 8 \mathrm{Vol} . \%$ MWCNT. 
Figure 9.2: SEM image of the ball milled powder composite with $\mathrm{Cu}-\mathrm{Cr} 12 \mathrm{Vol} . \%$ MWCNT.

Figure 9.3: SEM image of the ball milled powder composite with $\mathrm{Cu}-\mathrm{Cr} 14 \mathrm{Vol}$. \% MWCNT.

Figure 9.4: SEM and EDS analyses of the Cu-Cr 16 Vol. \% MWCNT composite 72

Figure 9.5: Low magnification SEM morphology images of $\mathrm{Cu}-\mathrm{Cr} 15 \mathrm{wt} \%$. 73

Figure 9.6: SEM image showing pores of $\mathrm{Cu}-\mathrm{Cr} 15$ wt. \% composite sintered at $750{ }^{\circ} \mathrm{C}$

Figure 9.7: EDS analysis of $\mathrm{Cu}-\mathrm{Cr} 15 \mathrm{wt} . \%$ compact sintered at $750{ }^{\circ} \mathrm{C}$ 74

Figure 9.8: Mapping of $\mathrm{Cu}-\mathrm{Cr} 15$ wt. \% compact sintered at $750{ }^{\circ} \mathrm{C}$ illustrating homogeneity of the composite.

Figure 9.9: SEM image showing MWCNT dispersion in $\mathrm{Cu}-\mathrm{Cr} 8 \mathrm{Vol}$. \% MWCNT compact sintered at $750^{\circ} \mathrm{C}$, illustrating the dispersion of MWCNT. 75

Figure 9.10: Mapping analysis for $\mathrm{Cu}-\mathrm{Cr} 8 \mathrm{Vol}$. \% MWCNT composite sintered at $750{ }^{\circ} \mathrm{C}$. 76

Figure 9.11: SEM photomicrograph of Cu-Cr 10 Vol. \% MWCNTs sintered at $750{ }^{\circ} \mathrm{C} .76$ Figure 9.12: SEM image of $\mathrm{Cu}-\mathrm{Cr} 12 \mathrm{Vol} . \%$ MWCNT composite sintered at $750{ }^{\circ} \mathrm{C} \ldots 77$ Figure 9.13: EDS data of $\mathrm{Cu}-\mathrm{Cr} 12 \mathrm{Vol} . \%$ MWCNT composite sintered at $750{ }^{\circ} \mathrm{C} \ldots \ldots . .77$ Figure 9.14: Mapping of $\mathrm{Cu}-\mathrm{Cr} 12 \mathrm{Vol} . \%$ MWCNT composite sintered at $750{ }^{\circ} \mathrm{C} \ldots \ldots . . .79$

Figure 9.15: SEM analyses of $\mathrm{Cu}-\mathrm{Cr} 14 \mathrm{Vol}$. \% MWCNT composite sintered at $750{ }^{\circ} \mathrm{C}$

Figure 9.16: Elemental Mapping analysis of $\mathrm{Cu}-\mathrm{Cr} 14$ Vol. \% MWCNT composite sintered at $750{ }^{\circ} \mathrm{C}$.

Figure 9.17: SEM image for $\mathrm{Cu}-\mathrm{Cr} 16 \mathrm{Vol}$ \% MWCNTs, 81

Figure 9.18: Mapping analysis of $\mathrm{Cu}-\mathrm{Cr} 16 \mathrm{Vol} . \%$ MWCNT sintered at $750{ }^{\circ} \mathrm{C}$, that $\mathrm{Cu}-\mathrm{Cr}$ are homogeneously distributed, MWCNTs were not evenly dispersed. 82

Figure 9.19: Mapping analysis of $\mathrm{Cu}-\mathrm{Cr} 15$ wt. \% sintered at $900{ }^{\circ} \mathrm{C}$. 83

Figure 9.20: Mapping analysis of $\mathrm{Cu}-\mathrm{Cr} 8 \mathrm{Vol}$. \% MWCNT sample. 84 
Figure9.21: SEM image showing dispersion of MWCNTs in $\mathrm{Cu}-\mathrm{Cr} 10 \mathrm{Vol} . \%$ MWCNT pellet sintered at $900{ }^{\circ} \mathrm{C}$.

Figure 9.22: Mapping analyses of $\mathrm{Cu}-\mathrm{Cr} 10 \mathrm{Vol} . \%$ MWCNT composite sintered at $900{ }^{\circ} \mathrm{C}$.

Figure 9.23: SEM image showing CNT dispersion in $\mathrm{Cu}-\mathrm{Cr} 12$ Vol. \% MWCNT composite sintered at $900{ }^{\circ} \mathrm{C}$.

Figure 9.24: SEM image of $\mathrm{Cu}-\mathrm{Cr} 14 \mathrm{Vol} . \% \mathrm{MWCNT}$ composite sintered at $900{ }^{\circ} \mathrm{C} \ldots 86$

Figure 9.25: Mapping analysis showing the $\mathrm{Cu}, \mathrm{Cr}$ and MWCNT distribution in a $\mathrm{Cu}-\mathrm{Cr} 14 \mathrm{Vol}$. \% MWCNT composite sintered at $900{ }^{\circ} \mathrm{C}$.

Figure 9.26: SEM analysis of Cu-Cr $16 \mathrm{Vol} . \%$ MWCNT composite sintered at $900{ }^{\circ} \mathrm{C} .87$

Figure 9.27: SEM and Mapping analysis of $\mathrm{Cu}-\mathrm{Cr} 16 \mathrm{Vol}$. \% MWCNTs composite sintered at $900{ }^{\circ} \mathrm{C}$

Figure 9.28: SEM analysis of $\mathrm{Cu}-\mathrm{Cr} 15 \mathrm{wt} . \%$ composite sintered at $1050^{\circ} \mathrm{C}$ 88

Figure 9.29: Mapping analysis of $\mathrm{Cu}-\mathrm{Cr} 15 \mathrm{wt} . \%$ composite sintered at $1050^{\circ} \mathrm{C}$. 90

Figure 9.30: SEM image of $\mathrm{Cu}-\mathrm{Cr} 8 \mathrm{Vol}$ \% MWCNT composite sintered at $1050^{\circ} \mathrm{C} \ldots 90$

Figure 9.31: SEM images of $\mathrm{Cu}-\mathrm{Cr} 10 \mathrm{Vol}$ \% MWCNT composite sintered at $1050{ }^{\circ} \mathrm{C}$.

Figure 9.32: Mapping results of $\mathrm{Cu}-\mathrm{Cr} 10 \mathrm{Vol} . \%$ MWCNT composite sintered at $1050^{\circ} \mathrm{C}$ shows that the three phases are homogeneous....

Figure 9.34: SEM and Mapping analysis of $\mathrm{Cu}-\mathrm{Cr} 12 \mathrm{Vol}$. \% MWCNT composite sintered at $1050{ }^{\circ} \mathrm{C}$.

Figure 9.35: SEM images of Cu-Cr $14 \mathrm{Vol}$ \% MWCNT composite sintered at $1050{ }^{\circ} \mathrm{C}$

Figure 9.36: Mapping results of $\mathrm{Cu}-\mathrm{Cr} 14 \mathrm{Vol} . \%$ MWCNT composite sintered at $1050^{\circ} \mathrm{C}$.

Figure 9.37: SEM image of $\mathrm{Cu}-\mathrm{Cr} 16 \mathrm{Vol}$. \% MWCNT composite sintered at $1050{ }^{\circ} \mathrm{C} .95$

Figure 9.38: Mapping results of $\mathrm{Cu}-\mathrm{Cr} 16 \mathrm{Vol}$. \% MWCNT composite showing homogeneous distribution. 96

Figure 9.39: TEM photomicrograph of $\mathrm{Cu}-\mathrm{Cr} 10 \mathrm{Vol} . \%$ MWCNT composite sintered at $750{ }^{\circ} \mathrm{C}$. 
Figure 9.40: TEM photomicrograph of $\mathrm{Cu}-\mathrm{Cr} 10 \mathrm{Vol}$. \% MWCNT composite sintered at $900{ }^{\circ} \mathrm{C}$.

Figure 10.1: Variation of composite relative density with CNT volume content [76].... 99

Figure 10.2: Schematic of packing arrangement of CNTs, $\mathrm{Cu}$ and $\mathrm{Cr}$ particles in the $\mathrm{Cu}-\mathrm{Cr}-\mathrm{MWCNT}$ composite.

Figure 10.3 shows the variation of the density of unsintered and sintered composites as a function of temperature and CNT content.

Figure 10.3: Density versus Vol. \% MWCNT plot. .................................................. 103

Figure 10.4: Volume shrinkage Vs Vol. \% MWCNT. ................................................ 104

Figure 10.5: Linear shrinkage Vs Vol. \% MWCNT.................................................... 105

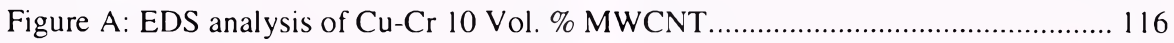

Figure B: EDS analysis of $\mathrm{Cu}-\mathrm{Cr} 10 \mathrm{Vol}$ \% MWCNT............................................. 117

Figure C: EDS analysis of $\mathrm{Cu}-\mathrm{Cr} 12$ Vol. \% MWCNT............................................. 118

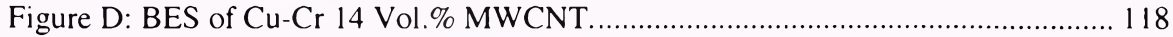

Figure E: EDS of Cu-Cr 14 Vol. \% MWCNT....................................................... 119

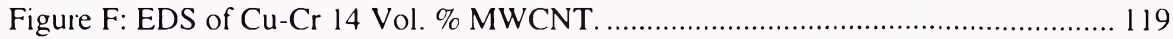

Figure G: EDS analysis of Cu-Cr 16 Vol. \% MWCNT ............................................. 120

Figure H: EDS analysis of Cu-Cr 16 Vol. \% MWCNT.......................................... 121

Figure I: EDS analysis of $\mathrm{Cu}-\mathrm{Cr} 8 \mathrm{Vol} . \%$ MWCNTcompact sintered at $750{ }^{\circ} \mathrm{C} \ldots \ldots \ldots 122$ 


\section{LIST OF NOMENCLATURE}

\begin{tabular}{|c|c|c|}
\hline Symbol & DEFINITION & SI UNITS \\
\hline $\mathrm{Ar}$ & Argon & -- \\
\hline$\overline{\mathrm{Cu}}$ & Copper & -- \\
\hline $\mathrm{Cr}$ & Chromium & -- \\
\hline$\overline{\mathrm{CNT}}$ & Carbon Nanotubes & -- \\
\hline$\sigma$ or $\kappa$ or $\gamma$ & Conductivity & $\mathrm{S} / \mathrm{m}$ \\
\hline$\rho$ & Density & $\mathrm{g} / \mathrm{cm}^{3}$ \\
\hline$\varphi$ & Diameter & inch \\
\hline MWCNT & Multiwall Carbon Nanotubes & -- \\
\hline$P$ & Porosity & -- \\
\hline $\mathrm{R}$ & Resistivity & ohm-m \\
\hline $\mathrm{s}$ & Spacing between the probes & $\mathrm{cm}$ \\
\hline $\mathrm{t}$ & Thickness of the sample & $\mathrm{cm}$ \\
\hline$\alpha$ & Temperature Cocfficient & -- \\
\hline$\overline{\mathrm{T}}$ & Temperature & ${ }^{\circ} \mathrm{C}$ \\
\hline $\mathrm{Hv}$ & Vickers' Hardness & -- \\
\hline $\bar{W}$ & Weight & $\mathrm{g}$ \\
\hline
\end{tabular}




\section{LIST OF ACRONYMS}

\begin{tabular}{ll}
\hline amu & atomic mass unit (atomic weight) \\
\hline BPR & Balls-to-Powder Ratio \\
\hline CNT & Carbon Nanotubes \\
\hline CVD & Charge Ratio \\
\hline MMC & Chemical Vapor Deposition \\
\hline IACS & Metal Matrix Composite \\
\hline NTP & International Annealed Copper Standard \\
\hline PM & Normal Temperature Pressure \\
\hline SEM & Powder Metallurgy \\
\hline XRF & Scanning Electron Microscope \\
\hline rpm & X-ray Fluorescence \\
\hline MWCNT & rotations per minute \\
\hline HEBM & Full-Width at Half Maximum \\
\hline EDS & Multiwall Carbon Nanotubes \\
\hline FCHM meter & Energy Dispersive Spectrometer \\
\hline
\end{tabular}




\section{INTRODUCTION}

\subsection{General}

There is a great need for materials in the aerospace industry that possess good electrical, thermal conductivity, and mechanical integrity, for use as contact materials in Vacuum Circuit Breakers (VCB). In the 1970's, the copper (Cu) and chromium (Cr) alloy was developed for medium voltage and high current VCB applications [1, 2]. This alloy has served as the most common contact material in electrical systems. Although $\mathrm{Cu}-\mathrm{C} \mathrm{r}$ alloys are of great interest due to their good electrical and thermal conductivity in conjunction with high strength and ductility $[1,2]$, the usage of up to $50 \%$ of $\mathrm{Cr}$ (a strategic metal) in certain alloys is resource prohibitive. Subsequently, any reduction in the amount of $\mathrm{Cr}$ required without a compromise in the desired properties of the alloy is desirable.

Previously, researchers have focused their efforts on developing $\mathrm{Cu}-\mathrm{Cr}$ alloys with fine and uniform microstructure to enhance the physical properties. This investigation is focused on the development of a Cu-Cr-Multiwalled Carbon Nanotube (MWCNT) composite material that possesses enhanced electrical and mechanical properties as compared to the currently used materials.

\subsection{Background}

Many types of alloys possessing good conductivity have been prepared, such as $\mathrm{Cu}-\mathrm{Ag}, \mathrm{Cu}-\mathrm{Nb}$ and $\mathrm{Cu}-\mathrm{Fe}$ alloy. Some of these alloys have high strength and high electrical conductivity, but are expensive. Others. which have good strength, lack the desired electrical conductivity [2]. Focusing on the Cu-Cr alloy, researchers found that 
the usage of $\mathrm{Cr}$, a strategic metal is resource prohibitive. In order to reduce the amount of $\mathrm{Cr}$, a ternary element, MWCNTs is added. Theoretical and experimental studies on CNTs have brought to the fore their exceptional mechanical and electrical properties, as well as their ability to exhibit metallic or semiconducting behavior [3]. CNTs are a novel form of carbon that has attracted a lot of interest amongst researchers and various industries. Moreover, it has wide applications in nanoelectronic devices, field electron emitters, nanoprobes, nanocomposite materials amongst others [1, 2, 3]. CNT's possess many properties - from their unique dimensions to an unusual current conduction mechanism-that make them ideal components of electrical circuits. CNTs are recognized as the ultimate carbon fibers for high performance, multifunctional composites $[4,5]$. Considering these properties of CNTs, it was decided to use it as the ternary element for preparing a composite material suitable for vacuum circuit breakers.

Since its discovery in 1991, Iijima [6] CNTs have been the focus of considerable research due to their excellent mechanical, electrical, thermal and magnetic properties $[7$. 8]. Various methods have been used for the preparation of CNTs, such as chemical vapor deposition (CVD), arc discharge, laser ablation, solvothermal processing etc., and consequently mass production of CNTs with an acceptable price has been achieved $[9$. 10]. Theoretical and experimental results have shown superior electrical properties of CNTs with an electric-current-carrying capacity 1000 times greater than that of copper wires [11]. Therefore, CNTs have been found to be useful additives to improve electrical properties of composite materials. 
It was intended to develop a composite by adding a ternary clement, MWCNT in an effort to reduce the amount of $\mathrm{Cr}$ required in the $\mathrm{Cu}-\mathrm{Cr}$ alloy without compromising the electrical, thermal and mechanical properties. Cu and $\mathrm{Cr}$ have low mutual solubility, which decreases to near zero at room temperature [12]. In fact, the maximum solubility of $\mathrm{Cr}$ in $\mathrm{Cu}$ is 0.89 at. $\%(0.71 \mathrm{wt} \%)$ at $1350 \mathrm{~K}$. The equilibrium phase diagram of $\mathrm{Cu}$ and $\mathrm{Cr}$ reveals a $\mathrm{Cu}$-rich eutectic at 1.56 at. \% (1.25 wt \%) $\mathrm{Cr}$ at $1350 \mathrm{~K}$. which is characterized by the liq $\alpha \mathrm{Cu}+\beta \mathrm{Cr}$ phase transformation. Below the eutectic isotherm. several microstructures with dissimilar mechanical and kinetic properties are produced, namely, primary dendritic $\mathrm{Cr}$ crystals in $\mathrm{Cu}, \mathrm{Cr}-\mathrm{Cu}$ eutectic, solute $\mathrm{Cr}$ atoms in $\mathrm{Cu}$ as well as solute $\mathrm{Cu}$ atoms in $\mathrm{Cr}$ and $\mathrm{Cr}$ precipitates formed during aging in the $\mathrm{Cu}$ matrix. These micro-constituents are known to strengthen the hypereutectic alloys. Furthermore. the addition of MWCNT it was envisaged that would impart enhanced electrical conductivity and mechanical strength as reported by other researchers $[13-19]$.

The main objective of this research was to develop a cost effective process for producing an optimized VCB material that possesses enhanced properties as compared to currently used materials. In order to further improve the electrical, thermal and mechanical properties of VCB materials, many experimental attempts [1-3, 14-17] were devoted primarily to developing $\mathrm{Cu}-\mathrm{Cr}$ alloys with line and uniform microstructure by processes that were efficient and cost effective.

Other researchers tried to prepare the contact material by adding ternary elements such as silver, aluminum, magnesium etc, but failed either due to low strength of the alloy or the cost factor. Shambaugh et al. (2006), found that the composite fiber with 1 wt. \% of CNTs showed more than $50 \%$ increase in tensile strength over a fiber drawn 
from matrix material alone [20]. This substantial increase in strength was believed to be due to a uniform dispersion of CNTs in the matrix material [20]. Current research in our laboratory has revealed that the electrical conductivity of $\mathrm{Cu}$ can be increased by $50 \%$ by the addition of $12 \mathrm{Vol} . \%$ of MWCNT. Based upon these results, the addition of MWCNT to $\mathrm{Cu}-\mathrm{Cr}$ was expected to increase mechanical strength, electrical conductivity and to be cost-effective.

\subsection{Drawbacks of Technique}

The $\mathrm{Cu} / \mathrm{CNT}$ composites have been prepared by the powder metallurgy technique $[13,21-23]$ as it offers the possibility of obtaining a uniform product with a reduction in tedious and costly machining processes. However, this technology has certain limitations mainly related with the poor affinity between $\mathrm{Cu}$ and $\mathrm{CNT}$, which results in weak interfacial bonding with the consequent negative effect on the structural, mechanical and electrical properties of the material $[13,21,24]$.

There are two important processing issues in the fabrication of CNT reinforced Cu matrix composite materials - establishment of a homogeneous distribution of CNTs and high interfacial bond strength between CNT's and Cu matrix [25]. Furthermore, the difficulty in distributing CNTs homogeneously in a Cu matrix has been regarded as one of the impeding hurdles [26, 27, 28]. and researchers have focused on solving this problem [29]. Inferior mechanical properties were achieved due to the agglomeration of CNTs [30], which affects the degree of compaction. On the other hand, the CNTs could impede the fusion of composites during pressing and sintering [31], unless the optimum amount needed to fill the pores. There also exists the problem of the lack of control over alignment [32]. Additionally, structural damage of the CNTs is often a problem 
encountered in the manufacturing of Cu-CNT composites due to the high temperatures and highly reactive environments associated with the methods of forming metal matrices. Limited availability of nanotubes has also restricted research efforts. although this seems to have become less of a problem in recent years as more researchers produce their own nanotubes via catalyzed chemical vapor deposition (CVD) methods.

\subsection{Drawbacks of $\mathrm{Cu}-\mathrm{Cr}$ Alloy}

As previously mentioned, the mutual solubility between $\mathrm{Cu}$ and $\mathrm{Cr}$ is small, and it decays to near zero at room temperature. The $\mathrm{Cu}$ based composite usually follows two criteria; firstly, it aims at further improving the mechanical properties of conventional binary alloys without severely deteriorating their electromagnetic properties, secondly, moving from binary to ternary alloys helps in cost reduction. Maximum solubility of $\mathrm{Cr}$ in $\mathrm{Cu}$ is 0.89 at.\% $(0.71 \mathrm{wt.} \%)$ at a temperature of $1350 \mathrm{~K}$. The equilibrium phase diagram of $\mathrm{Cu}$ and $\mathrm{Cr}$ shown in Figure 1.1 reveals a $\mathrm{C}$ (1-rich eutectic at 1.56 at.\% (1.25 wt.\%) $\mathrm{Cr}$ and $1350 \mathrm{~K}$, which is characterized by the decomposition liq. $\alpha \mathrm{Cu} \div \beta \mathrm{Cr}$. At hypereutectic compositions the shape of the $\mathrm{Cu}-\mathrm{Cr}$ equilibrium phase diagram is determined by the reaction liq. $\mathrm{Cu}+\beta \mathrm{Cr}$. These thermodynamic boundary conditions influence the formation of four different micro-structural phases with dissimilar mechanical and kinetic behavior, namely, primary dendritic $\mathrm{Cr}$ crystals in the $\mathrm{Cu}, \mathrm{Cr}-\mathrm{Cu}$ eutectic, solute $\mathrm{Cr}$ atoms in the $\mathrm{Cu}$ as well as solute $\mathrm{Cu}$ atoms in the $\mathrm{Cr}$, and $\mathrm{Cr}$ precipitates formed during aging in the Cu matrix [33]. Chromium is a strategic metal, and its usage in alloys for hardening, imposes resource limitations. Another major drawback is that some erosion occurs on the surface of all Cu-Cr alloy contacts after standard 14 shots. 


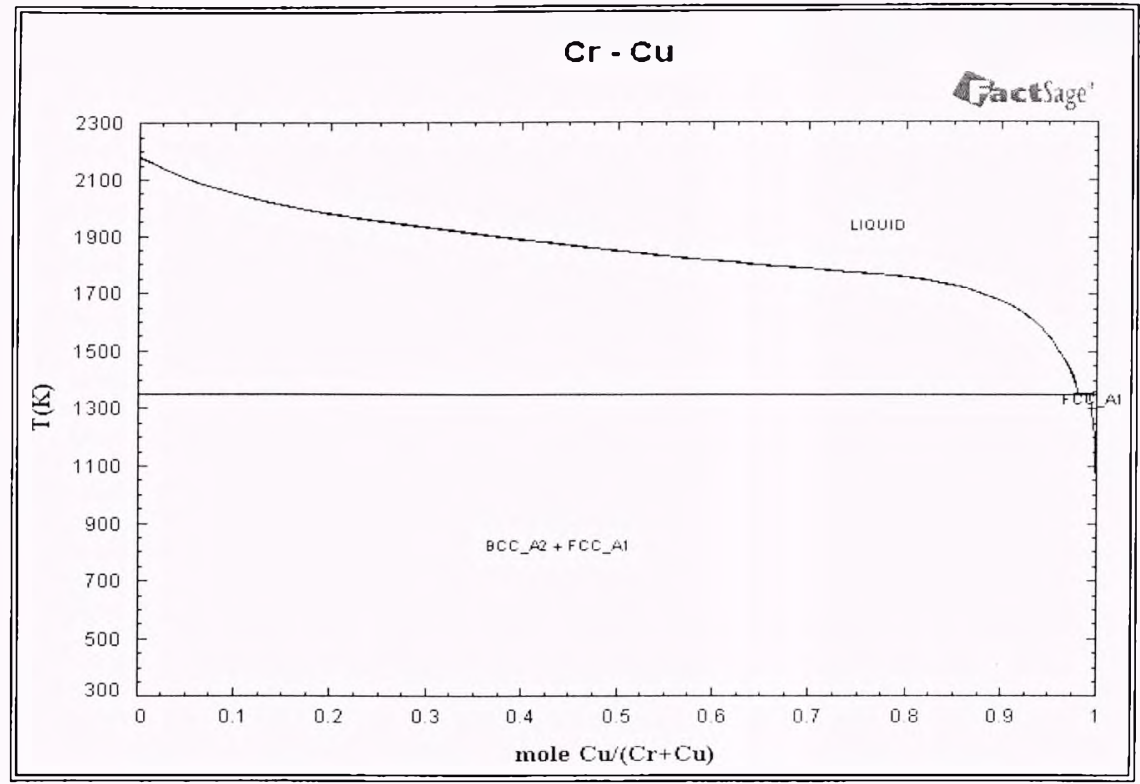

Figure 1.1: Cu-Cr phase diagram.

\subsection{Solution to the Problem}

\subsubsection{Effect of Content of CNT in Cu-CNT Composite}

The content of CNT in composites has had a significant effect on Vicker's hardness and the degree of compactness [31]. The Vicker's hardness increases with an increase in volume fraction of the $\mathrm{CNT}$. In the case of $\mathrm{Cu}^{\mathrm{U}} \mathrm{CNT}$ composite, maximum hardness is attained at $12 \mathrm{Vol} . \%$ [31]. The stress strain curve of C' $\mathrm{LI}$-CNT composites exhibited a tensile strength of 1.6 times greater than monolithic $\mathrm{Cu}[30]$. Also, it has been observed that the addition of CNT reduces the friction and weight loss as compared to that of pure Cu specimen [34]. 


\subsubsection{CNT Distribution}

The variation in diameter and the aspect ratio of CNTs create difficulty in obtaining a homogenous mixture. Additionally, damage to the walls of CNTs due to exposure to harsh conditions could further affect the homogeneity [35]. Some success has been achieved with conventional milling techniques, primarily with the use of low to moderate nanotube volume fractions [36 and 37]. In the current research, a high speed SPEX SamplePrep Mixer/Mill was used to fabricate the Cu-Cr-MWCNT composite in an inert atmosphere. In an effort to minimize damage to the walls of CNTs small balls and a lower BPR could be used in the ball milling process.

\subsubsection{Interfacial Bonding}

A significant increase in temperature occurs in the small metal powder particles during ball milling, due to their high surface energy. During the sintering process, semimolten $\mathrm{Cu}$ covers the surface of CNTs. Additionally. $\mathrm{Cr}$ reacts with CNTs producing carbides. The temperature, though instantancous, is sufficient to cause inter-diffusion and some degree of alloying. It should be noted that the addition of a substance to improve one property may have unintended effects on other properties. Thus, adding small amounts of CNT to an alloy such as Cu-Cr may increase or reduce its strength, hardness. electrical and thermal conductivity, corrosion resistance, or change its color.

$\mathrm{Cu}$ and $\mathrm{Cr}$ have FCC and BCC structure respectively, which can impose strain fields in the structure of the composite. However. $\mathrm{Cr}$ is thermodynamically predicted to form carbides with CNTs, particularly at freshly broken surfaces at the ends of tibers and 
along fractured walls. This anchoring of $\mathrm{Cr}$ with $\mathrm{CNT}$ combined with the formation of solute $\mathrm{Cr}$ in the $\mathrm{Cu}$ matrix bode well for good interfacial bonding within the composite.

\subsection{Summarized Overall Research Plan}

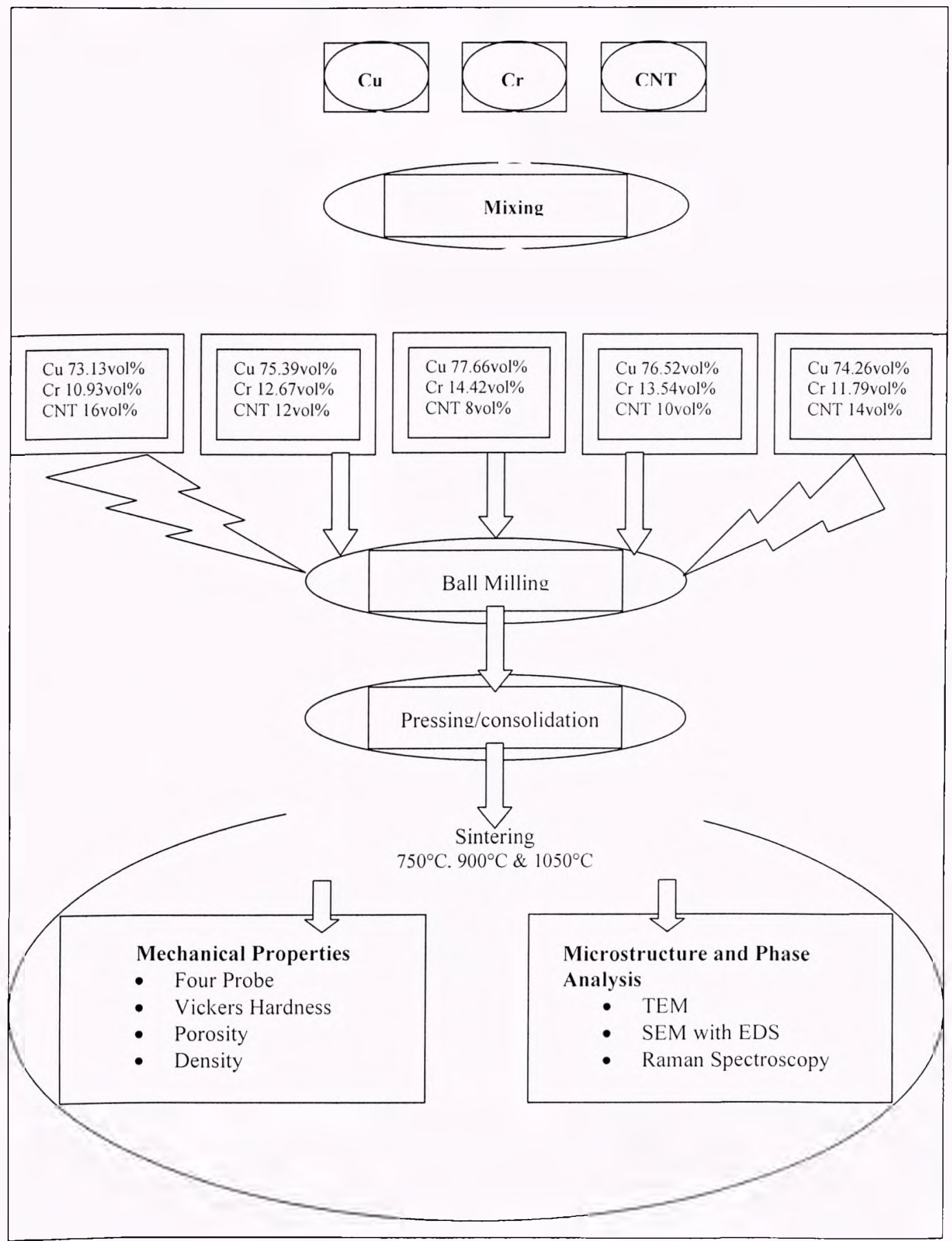




\section{MECHANICAL MILLING}

\subsection{Introduction}

Mechanical milling is a simple and useful technique to synthesize both equilibrium and non-equilibrium phases of commercially useful materials starting from elemental powders. It is also an economical process with important technical advantages. One of the greatest advantages of mechanical milling is in the synthesis of novel alloys that are not possible by any other technique, where normally immiscible elements are alloyed. This is because mechanical milling is a completely solid-state processing technique and therefore limitations imposed by phase diagrams do not apply.

Mechanical milling normally consists of a dry, high-energy ball milling technique, which has been employed to produce a variety of commercially useful and scientifically interesting materials. This technicue was developed during the late 1960s by John S. Benjamin and his colleagues at Paul D. Mercia Research Laboratory of the International Nickel Company (INCO) essentially to combine the advantages of precipitation hardening and oxide-dispersion strengthening in several nickel and ironbase superalloys [41-43].

The mechanical energy transferred to the powder particles in these mills takes place by shear action or impact of high velocity balls with the powder. It is considered as one of the most robust processing route for synthesizing a variety of materials.

During milling, solid-solid, solid-liquid and solid-gas reactions are initiated through repeated deformation and fracture of powder particles. Milling devices include planetary machines, where ball motion is generally chaotic. and vibrational mills, which may use either rods or balls. Milled products include amorphous, nanocrystalline and 
quasicrystalline materials [44-46]. In addition to size refinement. milling has received wide acceptability due to its potential to drive the changes at ambient conditions which are either not possible or require vigorous conditions in conventional processing routes [47-49].

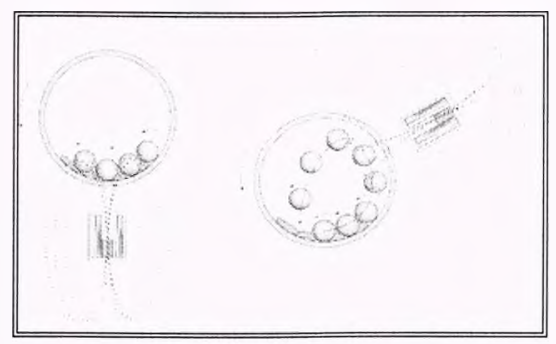

Figure 2.1: Mechanical milling [44].

\subsection{Important Attributes of Milling}

This simple but effective processing technique has been applied to metals, ceramics, polymers, and composite materials. The important attributes are [50]:

- Production of a fine dispersion of second phase (usually oxide) particles

- Extension of solid solubility limits

- Refinement of grain sizes down to the nanometer range

- Synthesis of novel crystalline and quasicrystalline phases

- Disordering of ordered intermetallics

- Possibility of alloying elements which are difficult to alloy

- Inducement of chemical (displacement) reactions at low temperatures

- Scaleable process 
Milling is employed mainly for particle size reduction through attrition and compressive forces at the grain size level. The ball milling machine that was used in the current research was the SPEX SamplePrep Mixer/Mill.

\subsection{Process of Milling}

The actual process of milling starts with mixing of the powders in the right proportion and loading the powder mix into the mill along with the grinding medium (generally steel balls). Normally, this is done in a glove box under an inert atmosphere. This mix is then milled for the desired length of time until a steady state is reached where every powder particle has the same composition in proportion of the elements employed.

\subsection{Raw Materials}

The raw materials used for milling are widely available as powders ranging in particle sizes from 1 to $200 \mu \mathrm{m}$ at various degrees of purity. The particle size of the powder is not critical, except that it should be smaller than the size of the grinding balls. because the powder size decreases exponentially with time and reaches a small value after a few minutes of milling. Occasionally, metal powders are milled with a liquid medium and this is referred to as wet grinding; if no liquid is involved then it is referred to as dry grinding. It has been reported that the rate of amorphization is faster during wet grinding than during dry grinding [51]. However, wet grinding has a serious disadvantage in that it is accompanied by increased contamination of the powder. Thus, most of the milling operations have been conducted in the dry mode.

\subsection{Process Variables}

Since mechanical alloying is a complex process, changes in the process variables would result in significantly different results. Hence, optimization of the process 
variables to achieve the desired product phase and/or microstructure is initially done and later the optimized parameters are maintained for consistency of the experimental results. Some of the important parameters considered in this rescarch are mentioned below:

\subsubsection{Type of Mill}

Most commonly used mills for mechanical alloying are SPEX shaker mills, Planetary ball mills, Attritor mills, Commercial mills etc. A suitable mill is generally chosen based on many desired factors such as type of powder, the quantity of powder. final constitution required etc. Generally, SPEX mills are most famous for laboratory research because of their user-friendly features, which include compactness, ease in operation, affordable price, and most importantly, the quickness in achieving the desired microstructure or phase. Table 2.1 summarizes the specifications of the SPEX mill.

Table 2.1: Specifications of SPEX mill

\begin{tabular}{|c|c|}
\hline Parameter & SPEX 8000 Mill \\
\hline Velocity of the ball & $<3.9 \mathrm{~m} / \mathrm{s}$ \\
\hline Kinetic energy & $<120 \times 10^{-3} \mathrm{~J} / \mathrm{hit}$ \\
\hline Shock frequency & $200 \mathrm{~Hz}$ \\
\hline Power & $<0.24 \mathrm{~W} / \mathrm{g} / \mathrm{b}$ all or rod \\
\hline
\end{tabular}

\subsubsection{SPEX SamplePrep Mixer/Mills}

SPEX SamplePrep Mixer/Mill is a variant of the ball mill or jar mill. in which powders are ground in a container along with one or more grinding elements. The containers are usually cylindrical; the grinding elements are most often balls but may be rods, cylinders, or other shapes. Generally the containers and grinding elements are made 
from the same material. As the container is rolled, swung. vibrated, or shaken, the inertia of the grinding elements causes them to move independently, into each other and against the container wall, grinding the sample. Variations of the "ball mill" range from laboratory mills with a sample capacity of a gram or less to large industrial mills with a throughput of tons per minute.

SPEX SamplePrep Mixer/Mills can be described as laboratory scale. high-energy ball mills (also known as high-energy shaker mills). They are designed to pulverize a sample rapidly while mixing it homogeneously — an approach long known to be elfective in preparing samples for emission spectroscopy and X-ray fluorescence (XRF) spectrometry. SPEX SamplePrep Mixer/Mills is also widely used for blending powders and making emulsions.

The shaking action of a SPEX SamplePrep Mixer/Mill is both complex and forceful. As the vial is swung back and forth in a shallow arc, its ends are displaced laterally. This distributes the grinding-ball impact over wide areas of the caps. In typical ball mills there are many grinding elements which travel only a short distance before impact. In the SPEX SamplePrep Mixer/Mill vials there are some balls develop high Gforces and travel the length of the vial at high velocity.

A distinct advantage of the SPEX Sample Prep Mixer/Mill design is the large selection of vial materials: hardened steel, stainless steel. tungsten carbide, alumina ceramic, zirconia ceramic, silicon nitride, agate, and plastic. Not only do these different media offer a wide range of grinding and blending performance, but they reduce contamination problems by allowing the analyst to avoid materials whose presence in the ground sample is objectionable. 


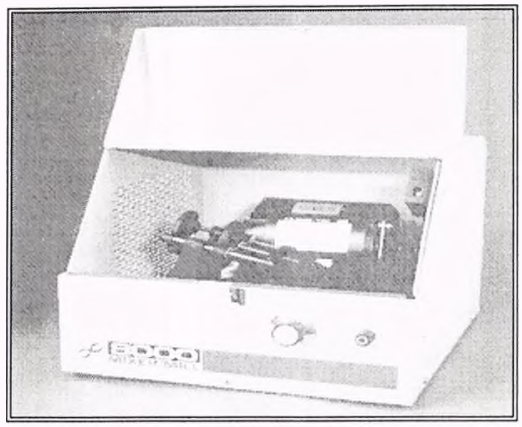

Figure 2.2: SPEX 8000 mixer / mill

\subsubsection{Milling Container}

Firstly, the material of the milling container should be chosen carefully to avoid contamination. Therefore, in milling the material of the container used for milling should be similar to that of the powder being milled. On the other hand, if both the milling container and the powder are not of the same material, the chemistry may be altered by the addition of the element from the milling container to the powder. Secondly, the shape of the container also plays an important role. Mainly, there are two types of SPEX mill containers being used: flat-ended and round-ended containers; of which the flat-ended has proven to be more effective due to the occurrence of significantly higher rates of alloying [52]. A hardened steel vial with stainless steel ball set can be seen in Figure 2.3. 


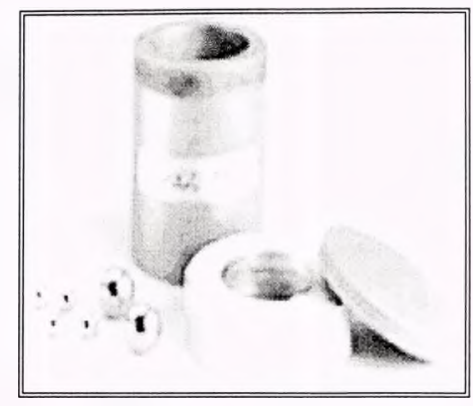

Figure 2.3: Hardened steel vial with stainless steel ball set.

\subsubsection{Milling Speed}

The higher the speed, the higher is the energy acting on the powder. However, higher speeds do not always result in the desired phase or microstructure formation. Furthermore, higher milling speeds increase the temperature of the vial t, which may be advantageous or disadvantages, again depending on the alloy system being used or the desired end phases [52]. Additionally, the high temperatures gencrated may also contaminate the powders. It has been reported that during nanocrystal formation, the average crystal size increases and the internal strain decreases at higher milling intensities due to the enhanced re-crystallization [52].

The standard SPEX 8000 mill has a fixed milling speed of about $1200 \mathrm{rpm}$. In the current research, in order to limit agglomeration and sticking effects, powder was scraped from the vial surface at regular intervals. A milling time circuit of 10 min (milling) +20 min (Idle time) was observed for every $10 \mathrm{~min}$ of milling to minimize the temperature rise during milling. For example, formation of an amorphous phase was achieved in at $\mathrm{Ti} \pm$ 
33 at. $\%$ Al powder mixture milled in a SPEX mill in $7 \mathrm{~h}$ at a BPR of $10: 1$, in $2 \mathrm{~h}$ at a BPR of $50: 1$ and in $1 \mathrm{~h}$ at a BPR of 100:1 [52].

\subsubsection{Milling Duration}

The duration of milling is considered to be the most important factor, for it mainly controls the desired end phase. Normally, the time is chosen so as to achieve a steady state between the fracturing and cold welding of the powder particles. Since all the milling parameters are interdependent, the milling time required depends on the type of the mill being used, intensity of the milling, charge ratio, temperature of milling etc. But, it should be realized that an increase in contamination and formation of undesired phases would occur if the powder is milled for longer times than required [52]. Hence, it is very essential to determine the optimum milling time for a mill with selected parameters. The effect of BPR and milling time is depicted in Figure 2.4.

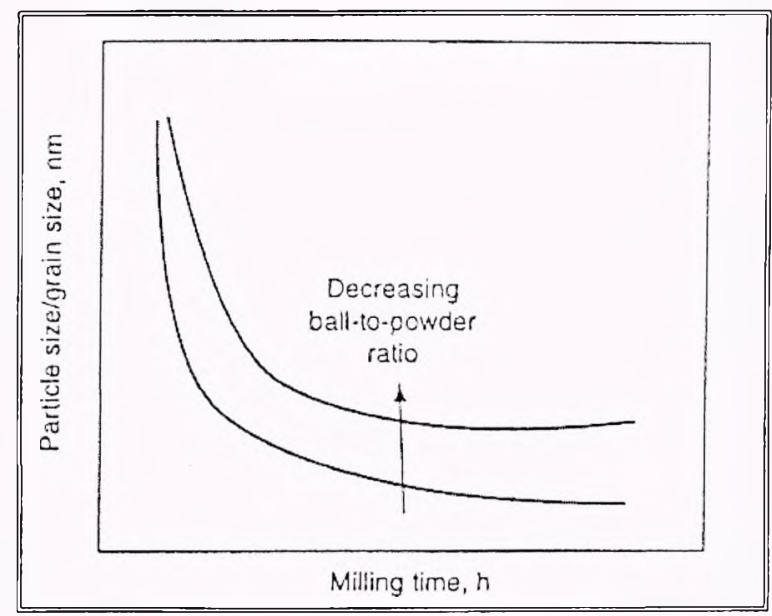

Figure 2.4: Refinement of particle and grain sizes with milling time and BPR [49]. 


\subsection{Grinding Medium}

Usually, a variety of steels such as hardened steel, tool steel, hardened chromium steel, tempered steel, stainless steel, bearing steel and WC-Co are the materials commonly used for grinding media/balls. The type of material chosen again depends on the powder mixture being milled. The size of the grinding medium has a greater impact on the milling efficiency of the process. Intuitively, one may assume that a larger size of the grinding medium (severe milling conditions), since it imparts more impact energy on the powder particles, is a favorable parameter. But experimental results suggest that mild milling conditions (small ball sizes, lower energies, and lower BPRs) seem to favor amorphization or metastable phase formation. Usually steel balls are very effective due to their high density and low contamination of the material being processed; stainless steel balls are unsuitable for some applications, including black powder and other flammable materials, which require non-sparking lead antimony, brass, or bronze grinding media.

\subsection{Charge Ratio}

The ratio of weight of balls to the powder being milled is termed the charge ratio (CR) or balls-to-powder ratio (BPR). This is one of the most important parameters during milling. The BPR is dependent on the type of mill being used. Usually a BPR of $10: 1$ is employed when a small capacity mill like the SPEX mill is being used. The BPR has a significant effect on the milling time required to achieve a particular phase. Generally. the higher the BPR, the higher is the impact energy, and the shorter is the time required. But there is a disadvantageous possibility of an increase in temperature leading to the crystallization of the amorphous phase, thereby resulting in non-desirable phases. Additionally, an increase of impact energy tends to destroy some materials such as CNTs, 
which could be detrimental to the intended property to be imparted to the composite. In the current research low BPR with shorter milling time were employed to mix the composite elements, and to ensure minimal destruction of CNTs. MWCNTs were used in this investigation, such that if some damage occurred to the CNTs, it would be primarily confined to the outer walls.

\subsection{Milling Atmosphere}

Milling process is generally conducted under vacuum or an inert atmosphere to prevent/minimize oxidation or contamination of the milled powder. Hence the selection and maintenance of a suitable milling atmosphere is of major importance since it has direct impact on the contamination of the powder. Again, its selection depends on the end products desired. Normally loading and unloading of the powder into the vial is carried out inside an evacuated glove box or controlled atmosphere. The glove box is continuously monitored to ensure an inert atmosphere is maintained [51]. It was observed that milling powders with different atmospheres results in a different constitution of the final powder.

\subsubsection{Powder Handling}

The powders of very small sizes require handling with caution and great care. Fine powders are highly reactive, sometimes pyrophoric and can be injurious when inhaled. Thus, precautions should be taken when opening the powder, especially immediately after milling since this can lead to its oxidation.

In this investigation, the milled powders were always removed in a glove box under argon allowing the vial to cool down to room temperature. before being stored in airtight glass containers. 


\subsubsection{Temperature of Milling}

Temperature at which the milling is conducted has a significant impact on the alloy phases formed, since a diffusion process is involved. Although the temperature of milling has a direct impact on milled powder, very few investigations have been conducted to measure the temperature, due to the complexities involved in monitoring the rapidly changing temperature. There have been conflicting reports on whether an amorphous phase or a nanocrystalline structure is formed with the increase or decrease in milling temperature [53].

\subsection{Milling Parameters Considered in this Research}

Type of mill SPEX 8000

Milling container hardened steel

Milling speed $1200 \mathrm{rpm}$

Milling time variable

Type, size and size distribution of the grinding medium.....stainless steel balls Ball-to-powder weight ratio approximately 10:1

Milling atmosphere argon

Process control agent none

Temperature of milling. room temperature

All these process variables are not completely independent. For example, the optimum milling time depends on the type of mill, size of the grinding medium, temperature of milling, ball-to-powder ratio, etc. Hence, they are carefully selected prior to the actual research. 


\subsection{Powder Contamination}

Powder contamination in mechanical milling is one of the most serious concerns. Some of the factors effecting the contamination of milled powders are fineness of the powder. milling atmosphere, material type of milling equipment. temperature increase during milling, milling time etc. During milling, proper care should be taken in order to prevent powder contamination. One of the most efficient methods to minimize or avoid contamination from milling atmosphere is to place the mill inside a chamber that has been evacuated and then filled with high-purity argon gas. If the milling container is not sealed properly, the outside atmosphere (mainly oxygen and nitrogen) could sneak in and contaminate the powder. Further, more the need of maintaining inert or suitable milling atmospheres is more emphasized by the fact that fresh surfaces, which are highly reactive, are formed as a result of collisions during the milling process. To minimize the contamination, it is always essential to use milling equipment (vial and balls) whose hardness is greater than that of the powder being milled. The fact that the metal powder coats the inner walls of the vial and the grinding medium during the initial stages of milling helps reduce contamination. It has been observed that milling of powder with ethanol under identical conditions reduces contamination [51].

Cross contamination could occur if the container that was previously used to mill one powder is used again to mill another powder without proper cleaning.

Measure to be taken to prevent powder contaminations are:

1. Milling tools harder than the powder to be milled.

2. Grinding media and the vials have the same chemical composition. 
3. Avoid using medium as this will increase cold welding and also can prevent the fracturing of the powder particles.

4. Minimizing the number of interruptions.

In general precautions should be taken so that the chemistry of the powder mixture is not compromised. Thus, contamination, unless special measures are taken to minimize/avoid it, seems to be an inherent drawback of the mechanical milling technique.

\subsection{Consolidation}

The consolidation of a powder is usually performed in a closed die, although other means such as roll compaction, iso-static compaction, extrusion or forging can be used. Parts can also be produced by slip casting using the techniques employed in pottery making. Regardless of the technique employed, each produces densification of the powder mass that can be related to the density of the solid metal at its upper limit [54]. In order to achieve uniform densification it is important to select a particle-size distribution which will permit uniform packing in the die. It is also necessary to select suitable pressing conditions as different materials require different compacting pressures. Compacting pressures that are too high will prevent proper sintering of the center of the compact and reduce the electrical conductivity and the strength.

Since mechanically milled powders possess high hardness due to work-hardening. cold compaction is not a very suitable consolidation approach. However, the purpose of this research was to develop and validate a novel technique to manufacture a $\mathrm{Cu}-\mathrm{Cr}$ MWCNT composite, hence this technique of consolidation was employed in this research. Cold compaction is a process wherein the powder materials are compressed in a temperature region where high temperature deformation mechanics like dislocation or 
diffusion creep arenot expected to occur. Cold compressing is the most important compaction method in powder metallurgy where small amounts of bulk powders, sometimes without lubricant or binder additions are compacted in a die [56]. This method is a simple method for producing high density pellets. Figure 2.5 and 2.6. illustrates components required for uniaxial cold compaction. The powder is loaded in the bore of the die where it is compacted by applying pressure uniaxially using a plunger. The compact density is mainly dependent on particle size, which influences packing density. and plasticity of the powders. Particle rearrangement occurs during compaction, wherein the particles rearrange themselves in the bore, when pressure is applied, without any deformation. The powders may exhibit both elastic and plastic deformations when pressure is applied.

In this investigation, an evacuable pellet die constructed of hardened stainless steel was used for the consolidation of the milled powders into pellets. The powder was uni-axially cold pressed at $844 \mathrm{MPa}(25,000 \mathrm{lbs}$ force) using a Carver manual pellet press and held at that pressure for about 90 minutes to form the pellet.

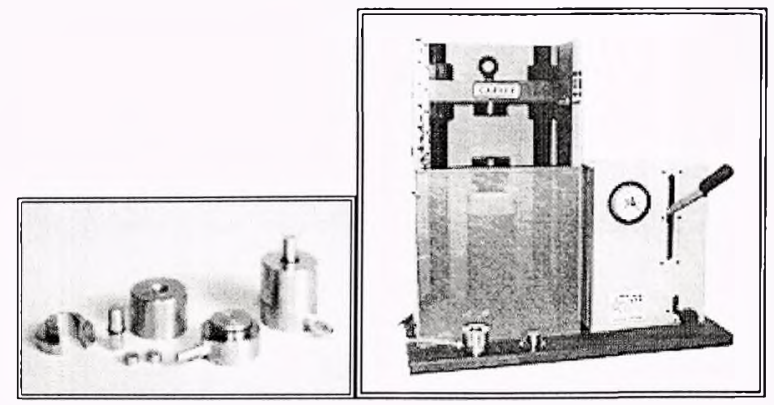

Figure 2.5: Die assembly and Carver press. 


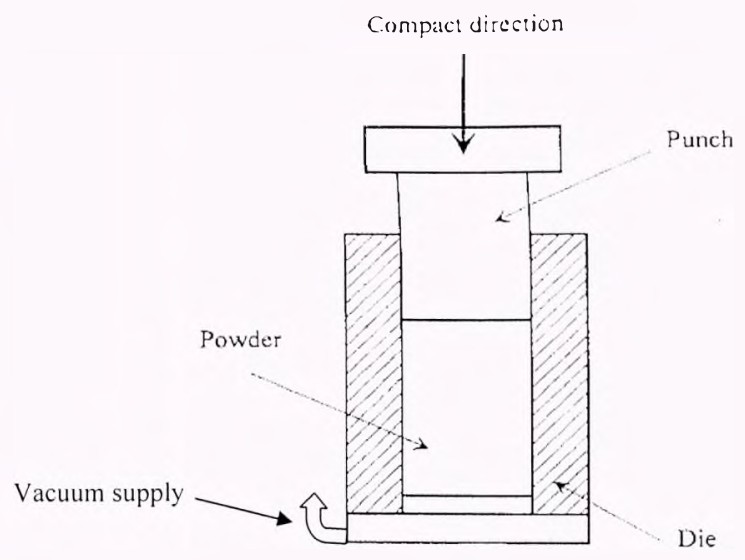

Figure 2.6: Uniaxial cold compaction

\subsection{Evaluation of Compaction Pressure}

The pressure applied by the Carver press was $25,000 \mathrm{lbs}$. In order to convert it into MPa the following calculations were performed.

The pellet diameter $=0.51 \mathrm{inch}$

The applied load $=25,000 \mathrm{lbs}$.

Pressure on the sample $(\mathrm{P})-\frac{\text { Force }}{\text { Area }}=\frac{F}{A}$

Where, $\mathrm{l}=25,000 \mathrm{lbs}$.

$$
\begin{aligned}
& A=\pi r^{2}=3.14 \times(0.51 / 2)^{2}=0.2041785 \text { inch. }^{2} \\
& P=25,000 / 0.2041785=122441.883 \mathrm{lbs} . / \text { inch. }^{2}=\underline{\mathbf{8 4 . 2 0 7 3} \text { MP il. }}
\end{aligned}
$$




\section{POROSITY \& DENSITY}

\subsection{Introduction}

Porosity is a measure of the void spaces in a material, and is measured as a fraction, between $0-1$, or as a percentage between $0-100$ percent. The term porosity is used in multiple fields including manufacturing, earth sciences and construction. The porosity of a material is determined by the total proportion of the air space or void space contained between the solid particles of which the body is composed [57]. Different materials have varying sizes which further results in spacing is known as pore spacing. It is calculated as the amount of water a pellet can hold.

The porosity affects the properties of materials to a great extend; such as absorption, resistance, conductivity, strength, etc. Since the aim of this research was the production of a composite material with high electrical conductivity and strength, the determination of porosity was of paramount importance.

According to E. W. Washburn there are six types of pores: (a) closed or 'sealed' pores; (b) channel pores connecting separate pores; (c) blind alley pores; (d) loop pores; (e) pocket pores-some with narrow necks; and (f) micro pores, which are so small as not to be filled with water or other liquid in any ordinary period of soaking [57].

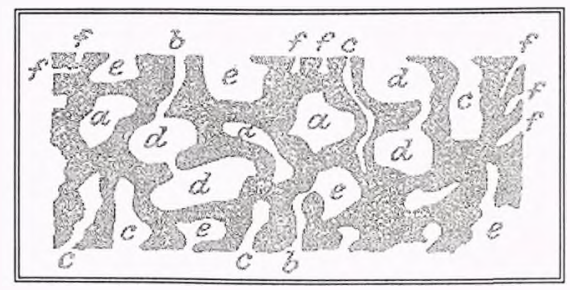

Figure 3.1: Various kinds of pores [57]. 
Due to these different kinds of pores, two sets of results are obtained when the porosity of a material is determined, viz.

(a) True porosity

(b) Apparent porosity

The true porosity is the ratio between the volume of all voids, both open and closed pores, and the total volume of the article.

The apparent porosity is defined as the ratio between the volume of water or liquid capable of being absorbed pores and the total volume of the article. Any closed pores, or for that matter, any open ones which are so fine that liquid cannot penetrate into them, will not be included as void space in determining the apparent porosity. Both the true and apparent porosity may be expressed as

(a) Percentage by weight

(b) Percentage by volume

The percentage of porosity by weight shows the weight of water or other liquid absorbed by 100 units of weight, whilst the percentage of porosity by volume is the volume of water absorbed in 100 volumes of the material or article [57]. It is customary to use the latter as this indicates the volume of the pores. The volume of the articles of the simple shape may be calculated from direct measurement of the dimensions, but where the shape is complex the volume must be determined by finding the difference between the weight when immersed in water and weight in air. The expression porosity by weight' is unsatisfactory because all the materials do not have the same density, so that the 'porosity' of different samples cannot be compared accurately. The expression of 
porosity wholly in terms of volume is, therefore. the most satisfactory as well as the most consistent measurement.

\subsubsection{Factors Affecting Porosity}

The porosity of various materials may be influenced by some or all of the following:

(a) Shape of the particles

(b) Size of the particles

(c) Grading of the particles

(d) Nature of the materials comprising the mixture

(e) Treatment to which the materials are subjected during manufacture and

(f) Relative position of the particle, i.e. whether they are closely compacted or lie loosely on one another.

Porosity can be reduced by carefully grading although in general, the coarser the maximum size of the particles the less will be the porosity. In other words an ideally well-graded mixture of fine particles docs not approach the theoretically-predicted minimum porosity tigure as closely as a coarser mixture. A mixture of angular grains readily compacts under pressure to give a low porosity. A lact which is often overlooked is that if the individual grains in a body are themselves porous. the body itself will have porosity higher than that of similarly graded mixture of non-porous particles. The porosity is also greatly influenced by the methods used for molding and the reactions which occur on firing. In some cases, a high porosity is desirable, particularly where a high thermal insulation is required. A large amount of air space entrapped within the 
particles of the body presents the maximum resistance to the passage of heat particularly if the voids are discontinuous.

\subsubsection{Measurement of Porosity}

As explained previously there is a distinction between the truc and apparent porosity, but in most cases the term porosity is applied to the apparent porosity as estimated from the amount of water or other suitable lluid absorbed by a given weight or volume of the sample. Hence the absorption or apparent porosity is a measure of the unsealed pores.

The usual way of carrying out the determination is to take conveniently sized test piece, which is weighed $\left(W_{1}\right)$ after being thoroughly dried. To ensure that liquid completely fills all unsealed pores the sample should be placed in a vacuum vessel, which is evacuated. Without releasing the pressure, the immersed liquid must then be introduced to such a level that the piece is entirely covered. The saturated sample may then be weighed in air $\left(\mathrm{W}_{2}\right)$ by suspending it from a thread to the arm of the balance; and finally the weight of the sample when immersed in water $\left(W_{3}\right)$ has to be measured.

The weight of the licuid absorbed is given by $W_{2}-W_{1}$ gram, provided that water (density $=1$ ) is used for immersion. The total volume of the test piece is $W_{2}-W_{3}$. i.e. the weight 'lost' on immersion in water, so the percentage apparcnt porosity by volume $(P)$ is given by the equation:

$$
P=\frac{W_{2}-W_{1}}{\left(W_{2}-W_{3}\right) \rho}
$$

Where, $\rho$ is the density of the liquid which will be I gram per cubic centimeter $\left(\mathrm{gms} / \mathrm{cm}^{3}\right)$ if water is used. 


\subsection{Density}

Density is defined as the mass of a unit volume of a substance. Density depends on the weight of the individual atoms and molecules making up the object. and how much space there is between them. Density also depends on whether the object is solid, filled with air pockets, or something in between. Densities are often given in terms of specific gravity. Expressed mathematically, Density $\left(\rho_{1}\right)=$ mass/volume In our work we used Archimedes Principal to calculate the density value.

Density $\left(\rho_{1}\right)$ is the density of pellet which is calculated by using:

$$
\rho_{1}=\frac{W_{1}}{W_{1}-W_{2}} \times \text { S. Giof werler }
$$

Where: $W_{1}$ is weight of the thoroughly dried sample and $W_{3}$ is the weight of the sample when immersed in water.

Feng Yi et. al. (2005), discussed the relative density of the silver-matrix composite reinforced by CNT. Figure 3.2, shows that when the CNT volume content was less than $8 \%$, the relative density of the composites increases with CNT and once the volume content is greater than $10 \%$, the relative density rapidly decreases.

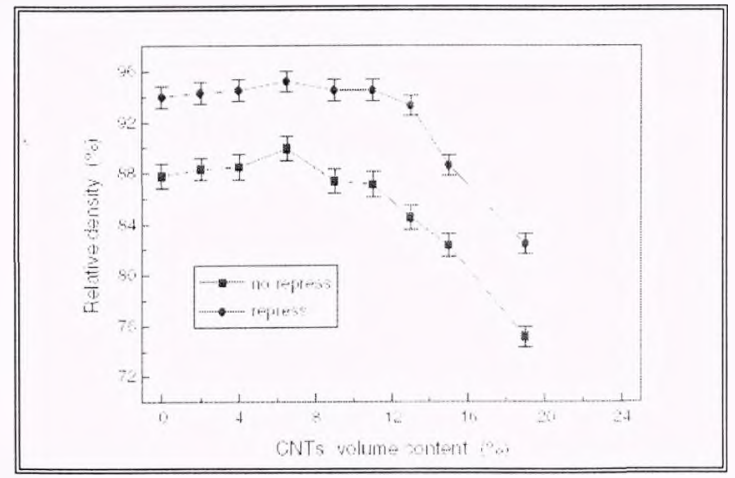

Figure 3.2: Variation of composite relative density with CNT volume content [76]. 


\section{ELECTRICAL CONDUCTIVITY}

\subsection{Introduction}

Electrical conductivity or specific conductivity is a measure of a material's ability to conduct an electric current. When an electrical potential difference is placed across a conductor, the movable charges (electrons) hlow and gives rise to an electric current. Conductivity is the reciprocal of electrical resistivity and has the SI units of siemens per meter $(\mathrm{S} / \mathrm{m})$ i.e. if the electrical conductance between opposite faces of a 1 meter cube of material is 1 siemens then the material's electrical conductivity is 1 siemens per meter.

\subsection{Effect of Temperature on Electrical Conductivity}

Temperature strongly affects the electrical conductivity, in metals, electrical conductivity decreases with increasing temperature, whereas in semiconductors. electrical conductivity increases with increasing temperature. In order to compare electrical conductivity measurements at different temperatures, they need to be standardized to a common temperature. This dependence is often expressed as a slope in the conductivityvs-temperature graph, and can be expressed as follows:

$$
\sigma_{1}=\frac{\sigma}{1+\alpha\left(T-T^{\prime}\right)}
$$

Where,

$\sigma_{\mathrm{T}^{\prime}}$ is the electrical conductivity at a common temperature. $T^{\prime}$

$\sigma_{T}$ is the electrical conductivity at a measured temperature, $\mathrm{T}$

$\alpha$ is the temperature compensation slope of the material

$\mathrm{T}$ is the measured absolute temperature

$T^{\prime}$ is the common temperature 


\subsection{Effect of Porosity on Electrical Conductivity}

Conductivity across a porous body takes place by five mechanisms all of which may contribute:

(a) Conductivity through the solid phase, which is of high valuc, but in a particulate system it will be limited by the points and surface area of contacts.

(b) The conductivity in the air space. which is low and for most purposes can be ignored.

(c) Radiational transmission across a void space which becomes significant only when there is a high temperature difference between two surfaces.

(d) Convection currents in the gaseous medium which depend on movement which can occur. This is greatest in masses with large open pores and is largely associated with hot bodies.

(e) Radiation transfer coefficient which determines whether radiation will be reflected from the surface or transmitted into the body itself.

Porosity introduces homogeneity in the bulk. which decreases the electrical conductivity of the composite. Presence of pores in solid not only significantly degrades the electrical characteristics but also the decreases the strength of the material. The principles of electrical insulation differ from thermal insulation in that ceramic and other non-metallic materials are relatively poor conductors of electricity so there is not the same requirement of high porosity [57]. The porosity in return is dependent on the milling parameters like milling time, BPR. size of the grinding medium and the method of pressing/consolidation. So it can be stated that also the parameters that influence the 
porosity of the composite indirectly influence the electrical conductivity of the composite. Therefore the milling parameters must be carefully chosen [59].

\subsection{Electrical Resistivity Measurement}

A four - point probe is used to measure the sheet resistance of a wide range of materials, from which the resistivity is calculated. The electrical conductivity is calculated as the reciprocal of resistivity (1/resistivity). IIP 4263A LCR meter (IFigure 4.1 and 4.2) illustrate the four - point probe meter with the measurement configuration, which permits easy, stable, and accurate measurements and avoids the measurement limitations due to factors such as mutual inductance. interference of the measurement signals, and unwanted residual in the test connections.

The schematic representation of the Four-point probe shown in Figure 4.2 shows four coaxial connectors:

1. $+\mathrm{I}: \mathrm{I} \mathrm{I}_{\mathrm{CUNR}}$ : High Current

2. $+\mathrm{V}: \mathrm{H}_{\text {pol }} \cdot$ Iligh Potential

3. $-V: L_{p}()$ r . Low Potential

4. -I : LCUR : Low Current

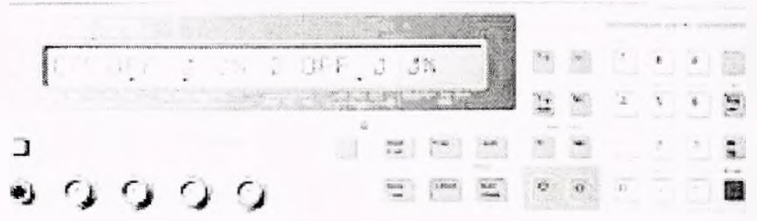

Figure 4.1: Four probe HP 4263A I.CR meter. 


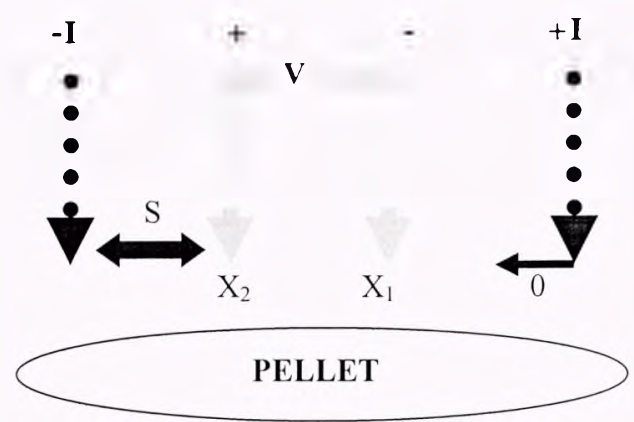

Figure 4.2: Schematic representation of the Four-point probe

The theory behind this method is based on a fixed current (I) which is injected into the material through the outer two probes, and a voltage $(V)$ is measured between the two inner probes. If the probes with uniform spacing s are placed on an infinite slab material of thickness $t$, then the resistivity $\rho$, is given by:

$$
\begin{gathered}
\rho=2 \Pi \mathrm{s} V / I \mu O h m-C e n t i m e t e r s \text { for } \mathrm{t} \gg \mathrm{s} \\
\rho=(\Pi \mathrm{t} / \mathrm{ln} 2) \mathrm{V} / \mathrm{I} \mu \mathrm{Ohm}-\mathrm{C} \text { entimeters for } \mathrm{s}>\mathrm{t}
\end{gathered}
$$

The four point probe measurement has the versatility in that it is useful for both low and high resistance measurements. The outer shield conductors work as the return path for the signal current measurement (they are not grounded). The same current flows through both the center conductors and outer shicld conductors (in opposite directions). so no external magnetic field is generated around the shield conductors (the magnetic fields produced by the inner and outer currents completely cancel each other). Thereforc. test leads do not contribute additional errors due to self or mutual inductance betwcen the individual leads. 


\subsection{Limits of Measurement Capability}

The probes must be able to make good ohmic contact with the material for accurate resistance measurement. In this regard Indium is used for good ohmic contact. Also, very low resistive materials (e.g. aluminum, gold, platinum) produce the maximum current. The current through the probe is restricted to $10 \mathrm{~mA}$ because of heating effects and excessive current density at the probe tips. An unclean sample or a sample that has surface doping will lead to inaccurate graphs due to an impeded ohmic contact or current leakage. The surface morphology also affects the results. In order to achieve the surface morphology between measurements, the sample surface is cleaned with sand paper. 


\section{VICKER'S HARDNESS}

\subsection{Introduction}

A Zhongguo HXD-100 TMC Shanghai Taiming Optical Instruments microhardness tester (200g load and $15 \mathrm{~s}$ dwell time) was used for estimating hardness of the samples. The unit of measure for hardness by the test is known as the Vickers Pyramid Number (HV). The hardness number can be converted into units of $\mathrm{Pa}$, but should not be confused with a pressure, which also has units of Pa. The hardness number is determined by the load over the surface area of the indentation and not the area normal to the force. and is therefore not a pressure.

\subsection{Vickers Test Procedure}

All Vickers ranges use a $136^{\circ}$ pyramidal diamond indenter that forms a square indent.

- The indenter is pressed into the sample by an accurately controlled test force.

- The force is maintained for a specific dwell time, normally $10-15$ seconds.

- After the dwell time is complete. the indenter is removed leaving an indentation on the sample that is squared in shape.

- The size of the indent is determined optically by measuring the two diagonals of the square indentation.

- The Vickers hardness number is a function of the test force divided by the surface area of the indent. The average of the two diagonals is used in the following formula to calculate the Vickers hardness.

$$
H V=(\text { Constant }) \times(\text { test force }) \text { indent diagonal squared }
$$


The constant is a function of the indenter geometry and the units of force and diagonal. The Vickers number, which normally ranges from HV 100 to HV 1000 for metals, will increase as the sample gets harder. Tables are available to make the calculation simple, while it is automatically calculated in all digital test instruments [59, $60]$.

The strengths of the Vicker's hardness test are:

1. One scale covers the entire hardness range.

2. A wide range of test forces to suit every application.

3. Nondestructive, sample can normally be used.
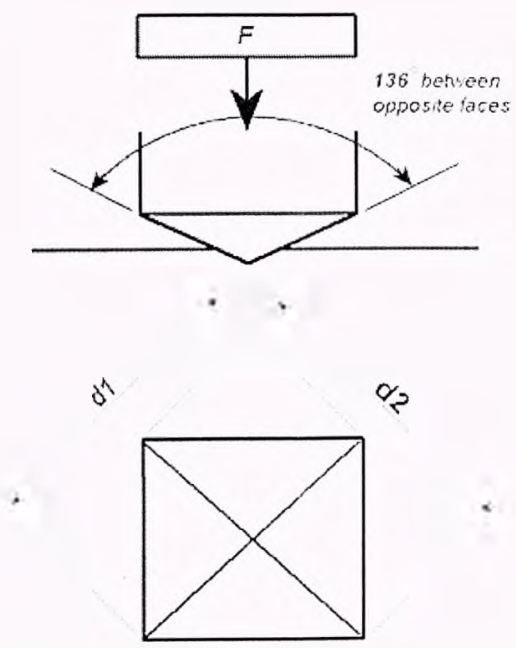

Figure 5.1: Schematic of Vickers hardness test [59]. 


\section{ANALYTICAL TECHNIQUES}

\subsection{Metallography}

In the Scanning Electron Microscope, electrons interact with the atoms that make up the sample producing signals that contain information about the sample's surface topography, composition and other properties such as electrical conductivity. The SEM images were used to assess the dispersion of CN'ts utilizing the Image-Pro imaging software. The quantitative image analysis illuminates the nature of CNT dispersion in the various treated powders.

Interaction of the primary beam with atoms in the sample causes shell transitions which result in the emission of X-ray energy. The emitted X-ray has an energy characteristic of the parent element. Detection and measurement of the energy permits elemental analysis (Energy Dispersive X-ray Spectroscopy) or EDS. X-rays may also be used to form maps or line profiles, showing the elemental distribution in a sample surface.

\subsection{Raman Spectroscopy}

Raman spectroscopy technique is used in condensed matter physics and chemistry to study the vibrational, rotational, and other low-frequency modes in molecular systems [61]. Raman spectroscopy relies on the monochromatic light, usually from a laser in the visible range. Here the laser light interacts with phonons or other excitations in the system, resulting in the energy of the laser photons being shilied up or down. The shift in energy provides information about the phonon modes in the system. 
Raman spectroscopy provides insight into structural, electronic and vibrational properties, of bonds within a material. The D Raman band of CNTs, which is observed between 1250 and $1450 \mathrm{~cm}^{-1}$, has a linear dependence on the laser excitation energy. This band is activated in the first-order scattering process of $\mathrm{sp} 2$ carbons by the presence of in-plane substitutional hetero-atoms, vacancies, grain boundaries or other defects and by finite-size [75]. The two strongest bands that occur approximately at $1330-1360 \mathrm{~cm}^{-1}$ and 1550-1600 $\mathrm{cm}^{-1}$ are called D-band and G-band respectively [62-67]. A plot of intensity of scattered light versus energy difference or shift is a Raman spectrum. 


\section{EXPERIMENTAL RESULTS}

\subsection{Pretreatment of Powder Feedstock}

The procedures adopted for the preparation of composite material are described in this section.

\subsubsection{As Received Cu, Cr and CNT Powder}

Cu metal powder (99.9\% pure, 1-5 Micron) and Cr metal powder (99.8\% pure, 1 5 Micron) were obtained from Atlantic Equipment Engineers, Bergentield, NJ. A JEOL Scanning Electron Microscopy (SEM) was used to determine the faceted morphology and particle size distribution of the as-received $\mathrm{Cu}$ and $\mathrm{Cr}$ powders. The Photomicrograph of each powder is shown in Figure 7.1.
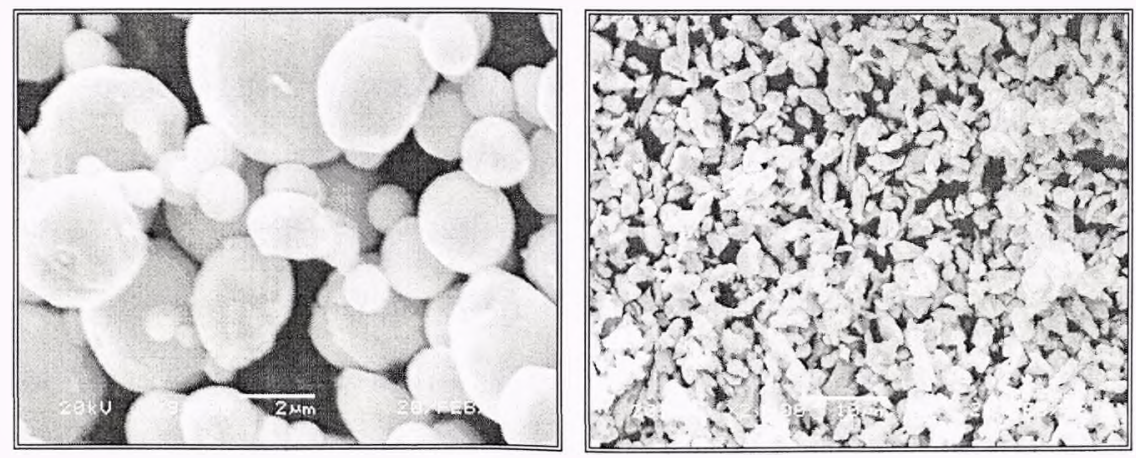

Figure 7.1: As-received Cu powder (lefi) and $\mathrm{Cr}$ powder (right) particles.

Multiwall carbon nanotubes (99\% pure, OD 40-60 nm) were obtained from Nanostructured \& Amorphous Materials Inc., Ilouston, TX. A CNT diameter of about 60 $\mathrm{nm}$ was observed in the TEM photomicrograph shown in. Figure 7.2 , with a wall 
thickness of $-25-30 \mathrm{~nm}$. It was observed that the MWCNTs were highly entangled and displayed a tendency to agglomerate.

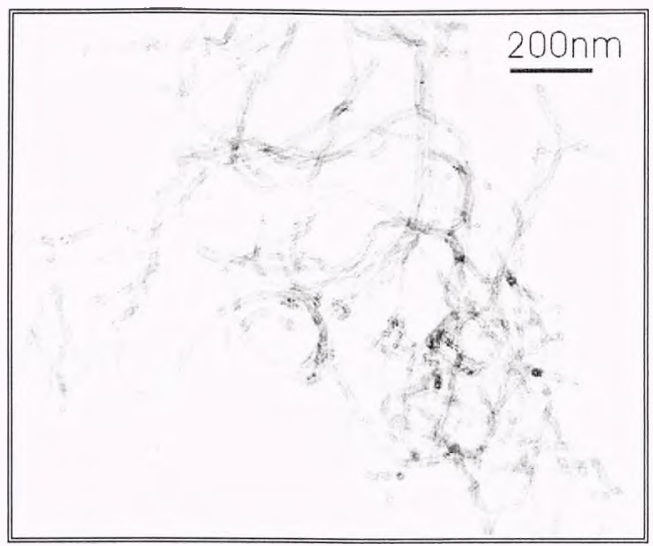

Figure 7.2: TEM of as received MWCNT powder.

\subsection{Selection of Appropriate Material and Milling Time}

The properties of composite materials are dependent on the appropriate selection of the materials and the proportion of each component. An important factor that affects the properties of composite materials is the particle size of its components, which affects the compactness, porosity, conductivity and strength. The particle size is dependent on the milling time, which inherently affects the porosity and density of the compact. In this investigation, ball milling was used to decrease the particle size of metal powders.

During ball milling, welding of particles. work hardening and fracture occur at different stages. In the early stage, the particles are soft. and thus weld together and form large particles. This agglomeration produces particles three times as large in size as compared with that of the starting particles. With continued deformation, the particles 
become work-hardened and fracture by a fatigue failure mechanism and/or by the fragmentation of fragile flakes. Fragments generated by this mechanism may continue to be reduced in size in the absence of strong agglomerating forces. Finally, after a certain period of milling time, a steady-state equilibrium is reached where a balance is achicved between the rate of welding, which tends to increase the average particle size, and the rate of fracture, which tend to decrease the average composite particle size.

SEM images of milled particles at different intervals of time were used to determine the particle size distribution, layer thickness and morphology with milling time. The figures 7.3-7.5 show the variation in particle morphology with increasing milling time.

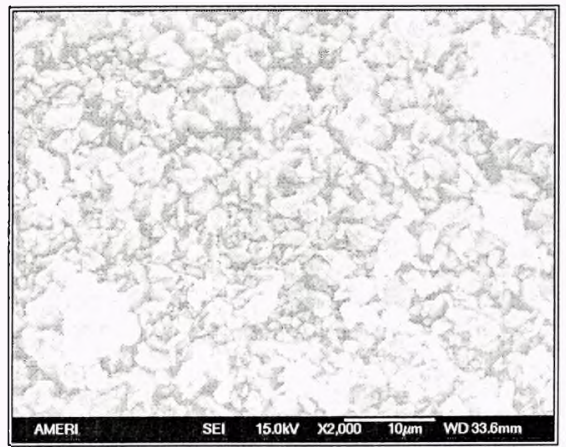

Figure 7.3: SEM of Cr particles after 15 minutes of ball milling.

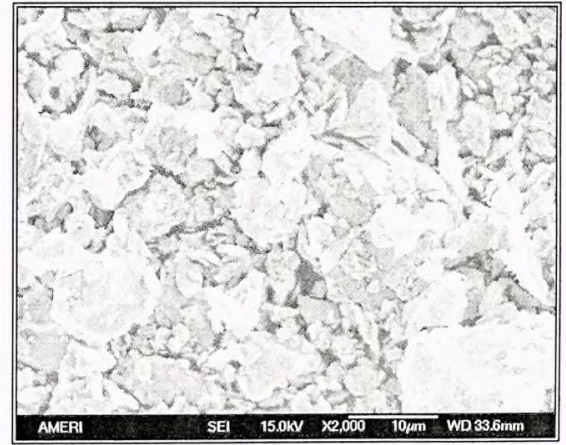

Figure 7.4: SEM of Cr particles after 45 minutes of ball milling. 


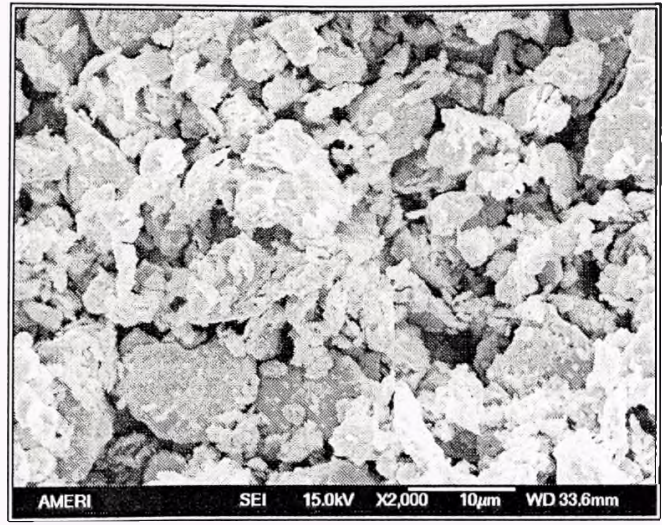

Figure 7.5: SEM of Cr particles after 60 minutes of ball milling

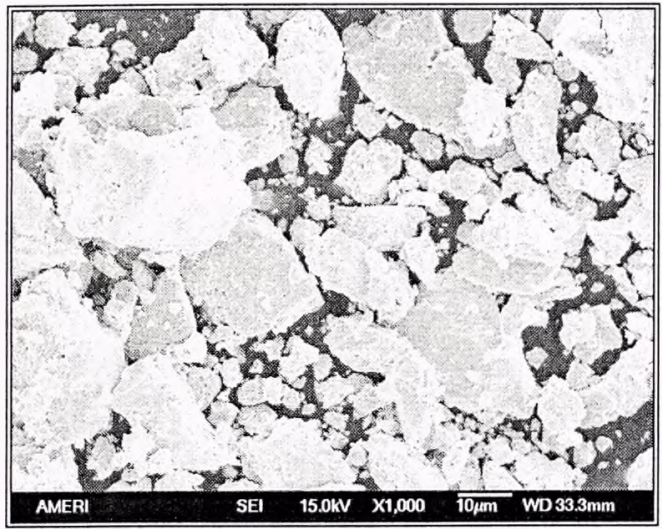

Figure 7.7: SEM of Cr particles after 180 minutes of ball milling

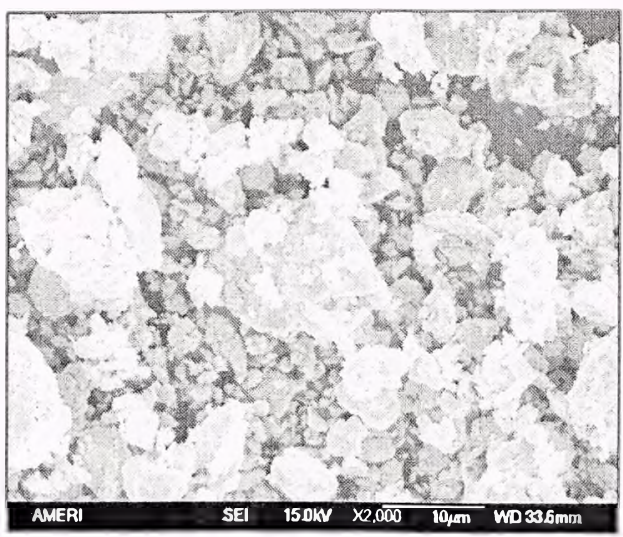

Figure 7.6: SEM of $\mathrm{Cr}$ particles after 120 minutes of ball milling.

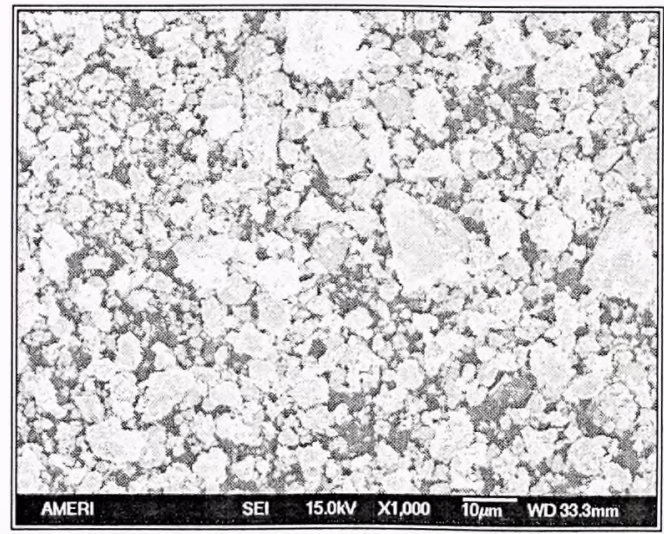

Figure 7.8: SEM of Cr particles after 240 minutes of ball milling.

The average diameter of powdered metal particles was plotted as a function of milling time. From the Figure 7.9, it can be observed that the particle size first increased in size due to agglomeration. 


\section{Cr Sample}

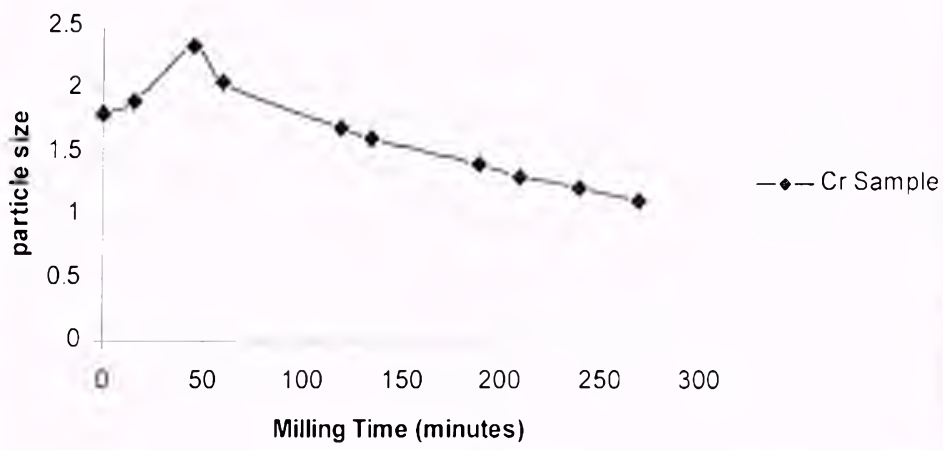

Figure 7.9: Plot of average particle size of $\mathrm{Cr}$ (micron) versus milling time (minutes).

Figure $7.10-7.17$ illustrate the change in morphology of $\mathrm{Cu}-\mathrm{Cr} 15$ wt. \% composite with milling time.

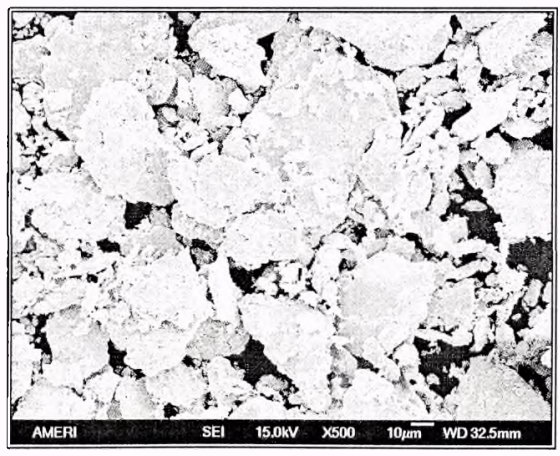

Figure 7.10: $\mathrm{Cu} \mathrm{Cr} 15$ wt. \% after 15 minutes of ball milling.

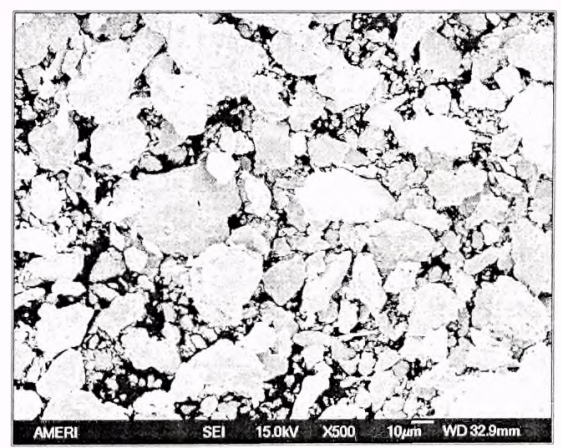

Figure 7.11: Cu Cr15 wt. \% after 30 minutes of ball milling. 


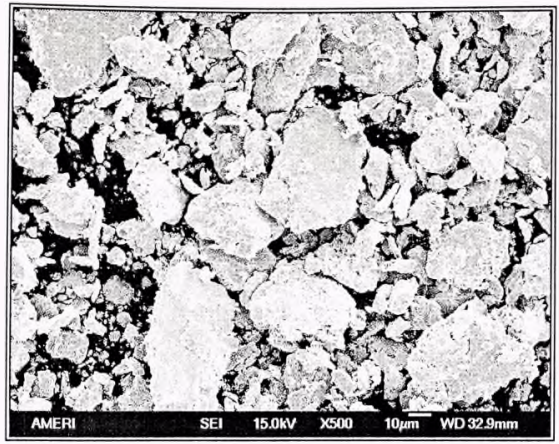

Figure 7.12: Cu Cr15wt. \% after 45 minutes of ball milling.

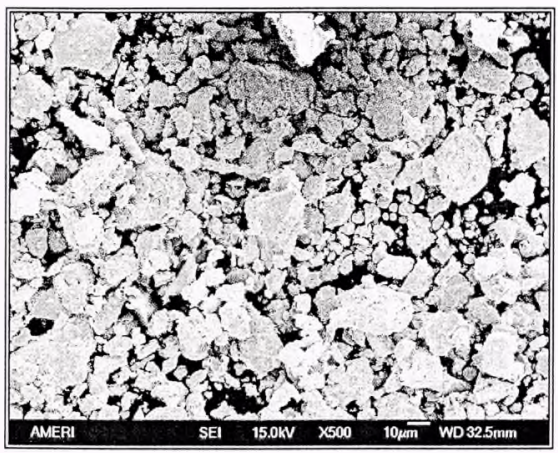

Figure 7.14: Cu C'rl 5wt. \% after 120 minutes of ball milling.

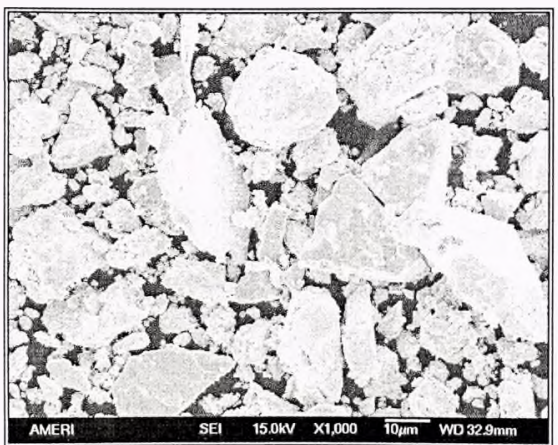

Figure 7.16: $\mathrm{Cu} \mathrm{Cr} 15$ wt. \% after 180 minutes of ball milling.

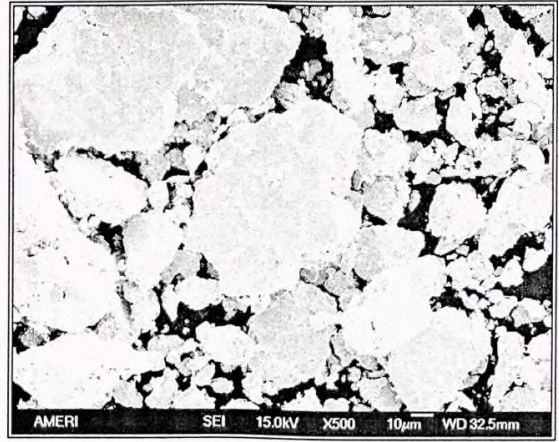

Figure 7.13: Cu Crl5 wt. \% after 60 minutes of ball milling.

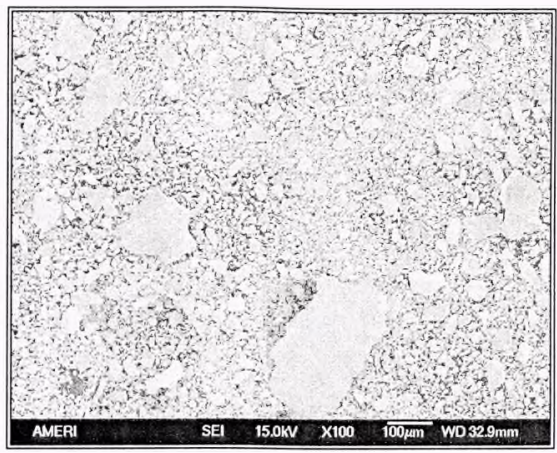

Figure 7.15: Cu Crl5wt. \% after 150 minutes of ball milling

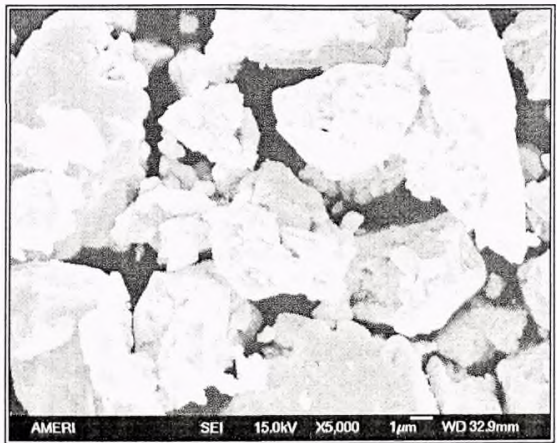

Figure 7.17: Cu Cr15wt. \% after 240 minutes of ball milling. 


\section{Cu Cr Sample}

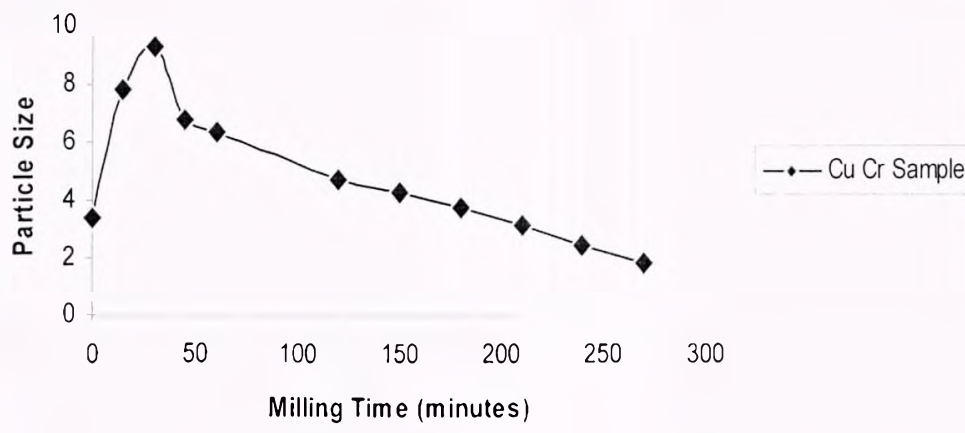

Figure 7.18: Plot of average particle size of $\mathrm{Cu}-\mathrm{Cr} 15$ wt. $\%$ composite versus milling time.

The SEM images (Figure 7.10-7.17) and the graph in Figure 7.18 revealed that the particle size of the milled powder did not decrease significantly after 240 minutes of milling.

\subsection{Addition of Ternary Element}

Many attempts [33] were made to add a ternary element to the $\mathrm{Cu}-\mathrm{Cr}$ alloy in order to enhance the properties of the material. As a consequence. new classes of ternary alloys were introduced with $\mathrm{Ag}, \mathrm{Mn}, \mathrm{Zr}$, etc. [33], as the ternary element with the intention to further improve the mechanical properties, and production cost. A ternary element allows the exploitation of a large variety of possible kinetic pathways for attaining certain flow stress-conductivity profiles [33]. In this rescarch, the amount of $\mathrm{Cr}$ normally present in the $\mathrm{Cu}-\mathrm{Cr}$ alloy was reduced by the addition of the temary element MWCNTs, with the intention of not compromising electrical, thermal and mechanical properties of the composite. 


\subsection{Electrical Conductivity of $\mathrm{Cu}$ and $\mathrm{Cu}-\mathrm{Cr}$ Alloy}

Table 7.1 summarizes the electrical conductivity of $\mathrm{Cu}$ and $\mathrm{C} u-\mathrm{Cr}$ alloy at various temperatures.

Table 7.1: Electrical conductivity

\begin{tabular}{|c|c|}
\hline Sample & Electrical Conductivity $\left(290^{\circ} \mathrm{C}\right)(\mathrm{S} / \mathrm{m})$ \\
\hline $\mathrm{Cu}$ (standard reference) & $5.96 \mathrm{E}+07$ \\
\hline Cu plate $(C-26000)$ & $5.93 E+07$ \\
\hline Cu pellet & $5.91 \mathrm{E}+07$ \\
\hline Cul-Crl5wt\% ${ }^{\mid 77]}$ & $4.07 \mathrm{E}+07$ \\
\hline $\mathrm{Cu}-\mathrm{Crl} 5 \mathrm{wt} \%\left(900^{\circ} \mathrm{C}\right)$ & $3.89 \mathrm{E}+07$ \\
\hline $\mathrm{Cu}-\mathrm{Cr} 15 \mathrm{wt} \%\left(750^{\circ} \mathrm{C}\right)$ & $3.55 \mathrm{E}+07$ \\
\hline Cu-Crl $15 w t \%$ (unsintered) & $3.09 \mathrm{E}+07$ \\
\hline
\end{tabular}

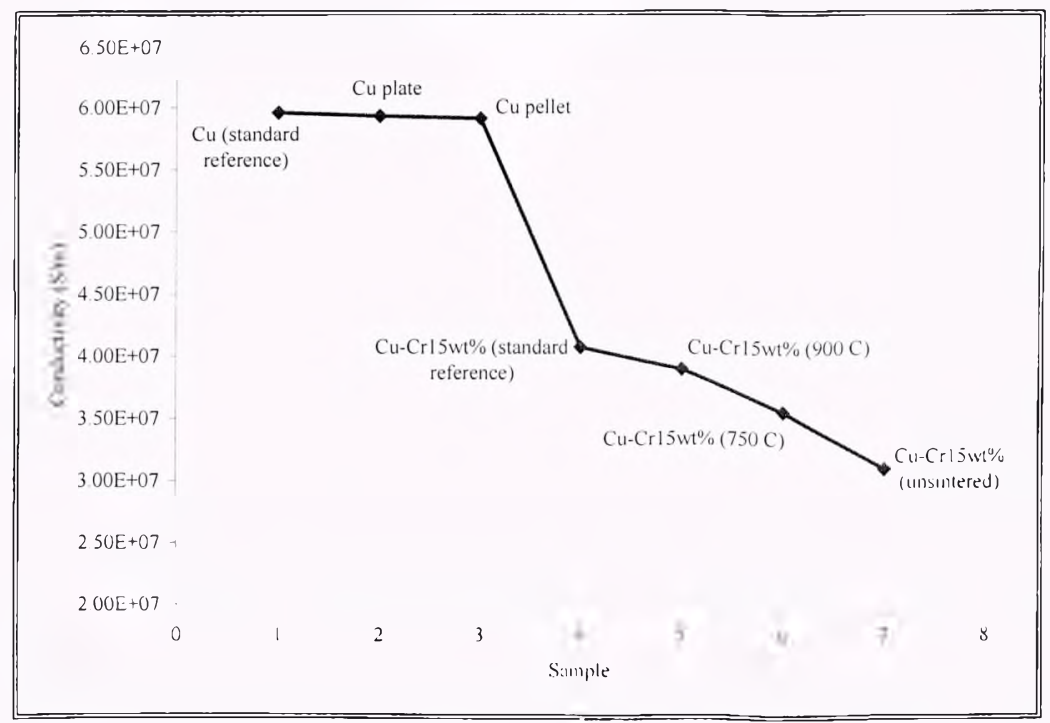

Figure 7.19: Electrical conductivity of $\mathrm{Cu}$ and $\mathrm{Cu}-\mathrm{Cr}$ alloys. 


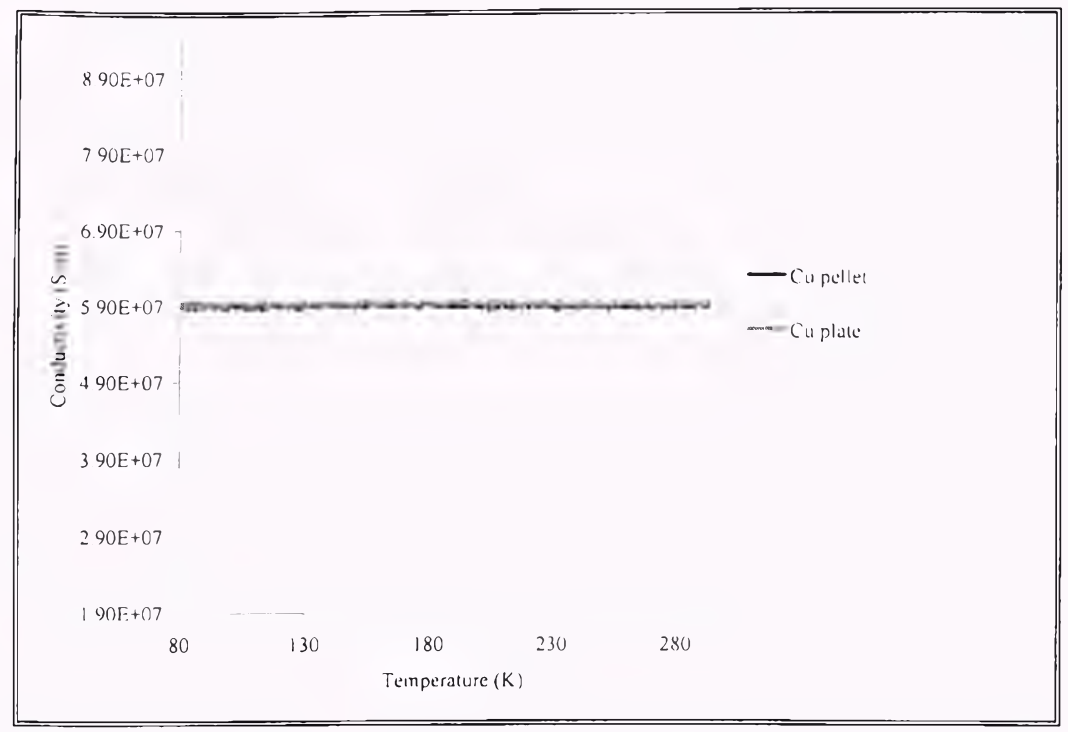

Figure 7.20: Conductivity versus temperature of $\mathrm{Cu}$ compact and Cu plate.

The electrical conductivity of pure copper depicted in Figure 7.20 confirmed the accuracy of the four probe resistivity meter. As shown in Figure 7.20 , the increase in slope with increase in the temperature is indicative of the metallic nature of Cu.

\subsection{Theory Behind the Electrical Conductivity of $\mathrm{Cu}-\mathrm{Cr}$}

According to the hard-sphere assumption, the atomic radius of pure C $\mathrm{u}$ is $\mathrm{R}_{\mathrm{Cu}}$ $0.128 \mathrm{~mm}$ and that of pure $\mathrm{Cr}$ is $\mathrm{R}_{\mathrm{Cr}}=0.125 \mathrm{~nm}$. This suggests that if the $\mathrm{Cr}$ solute dissolves into the $\mathrm{Cu}$ matrix, the $\mathrm{Cu}$ matrix lattice parameter will be reduced due to the smaller size of the $\mathrm{Cr}$ atom. Thus, a deviation in the value of the lattice parameter in the $\mathrm{Cu}$ matrix is good indicator of whether; $\mathrm{Cr}$ becomes part of the $\mathrm{Cu}$-matrix, or whether $\mathrm{Cr}$ precipitation occurs. It should be noted that $\mathrm{Cr}$ remains the dominant factor in the microstructure of the Cu-Cr alloy [77]. 


\subsection{Comparative Analysis}

As previously mentioned, a Cu plate (C26000) was used for comparative analysis and to assess the accuracy of the Four Probe meter. SEM and EDS analyses were conducted to determine the morphology and purity of the $\mathrm{Cu}$ plate. Figure 7.21 is a photomicrograph of the $\mathrm{Cu}$ plate. Figure 7.22 shows the EDS analysis of the Copper.

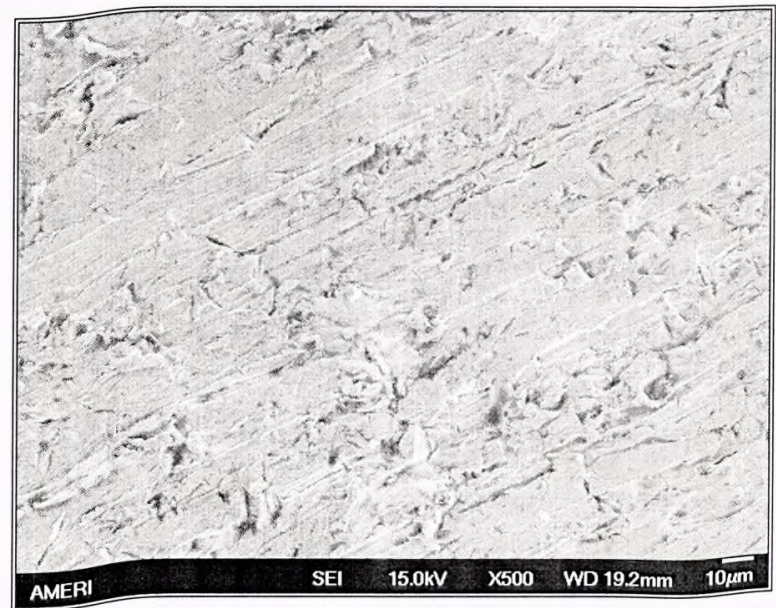

Figure 721: SEM analyses for pure C'u plate (i.e. (26000).

\begin{tabular}{|c|c|c|c|c|}
\hline $\begin{array}{l}\text { Element } \\
\text { Line }\end{array}$ & Weight \% & Atom \% & Formula & Compnel \% \\
\hline$C u K$ & 100.00 & 100.00 & $\mathrm{Cu}$ & 100.00 \\
\hline$C u L$ & $\cdots$ & $\ldots$ & & --- \\
\hline Total & 100.00 & 100.00 & & 100.00 \\
\hline
\end{tabular}




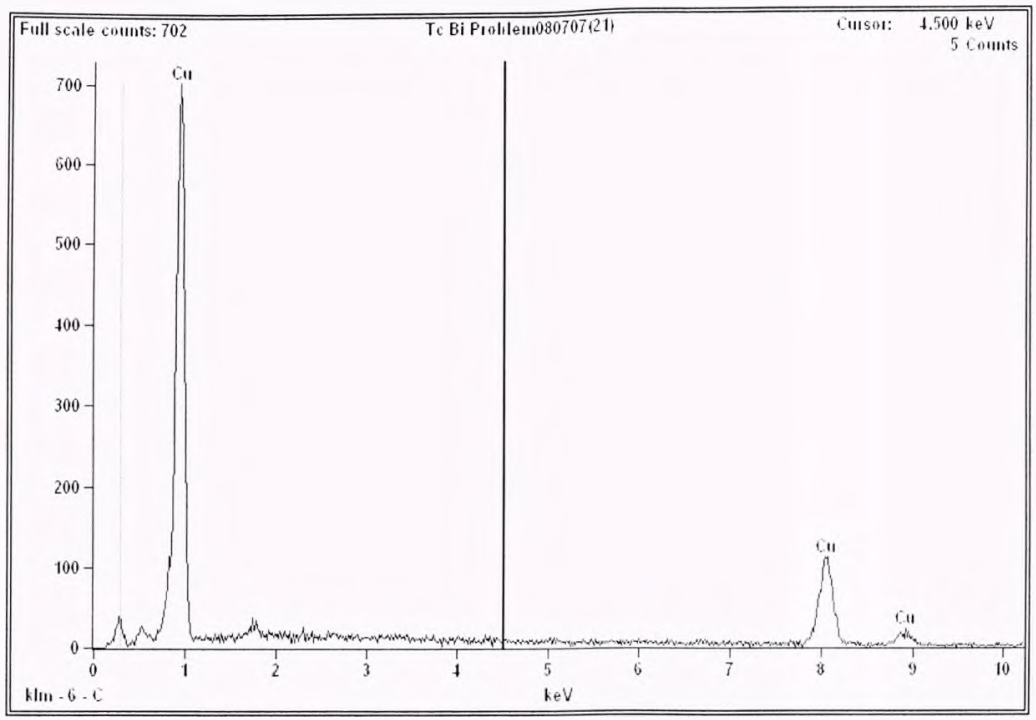

Figure 7.22: EDS analyses for pure Cu plate (i.e. C26000)

SEM and EDS analyses were conducted on as received CNTs to determine their purity. Additionally, the CNTs were sintered at $750 \mathrm{oC}, 900 \mathrm{oC}$ and $1050 \mathrm{oC}$ under inert atmosphere in order to determine the effect of temperature on their morphology. The results of SEM and EDS analyses indicated no significant change in surface morphology and composition with heating. Figure 7.23 - 7.30 SEM photomicrograph with EDS analysis of as received CNTs as well as sintered CNTs. 


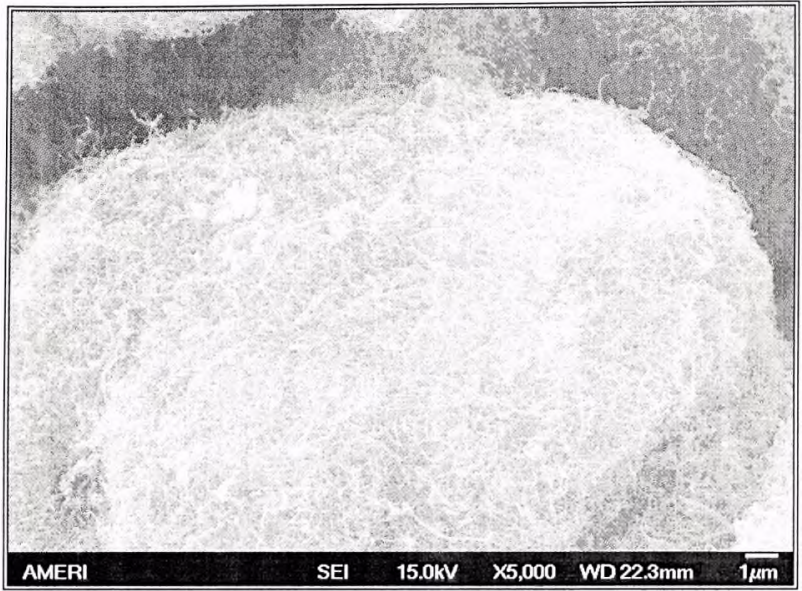

Figure 7.23: Pure CNTs as received.

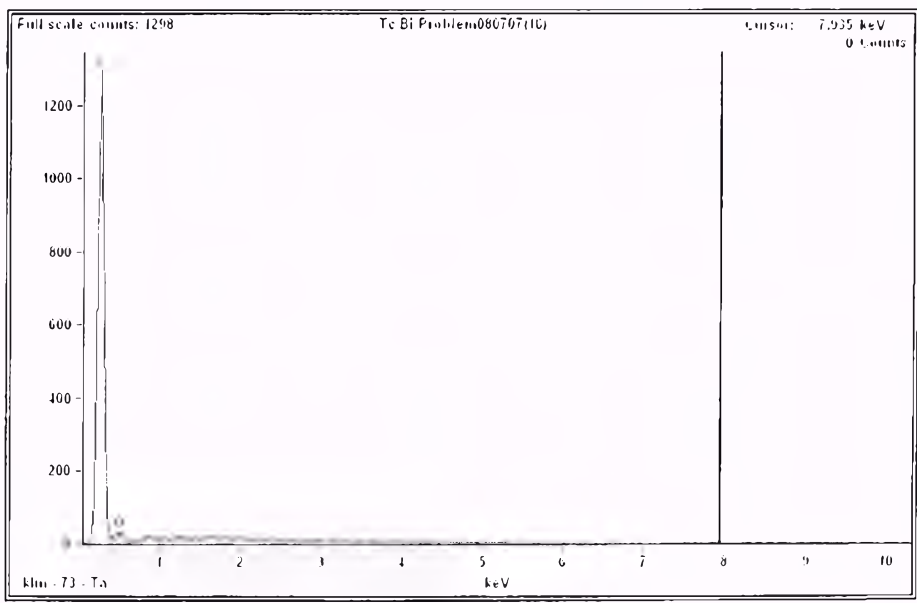

\begin{tabular}{|c|c|c|c|c|}
\hline Element Line & Weight $\%$ & Atom \% & Formula & Compnd \% \\
\hline$C K$ & 100.00 & 100.00 & $\overline{C \mathrm{C}}$ & 100.00 \\
\hline$O K$ & 0.00 & 0.00 & $\mathrm{O}$ & 0.00 \\
\hline Total & 100.00 & 100.00 & & 100.00 \\
\hline
\end{tabular}

Figure 7.24: EDS analyses of pure CNT's as received. 


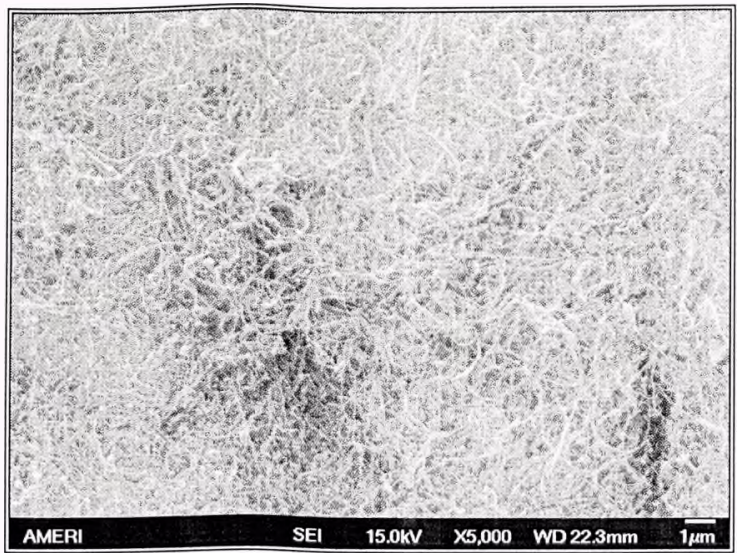

Figure 7.25: CNTs sintered at $750{ }^{\circ} \mathrm{C}$ in an inert atmosphere.

\begin{tabular}{||c||c|c|c|c||}
\hline \hline Element Line & Weight \% & Atom \% & Formula & Compnd \% \\
\hline \hline C K & 99.54 & 99.91 & $\mathrm{C}$ & 99.54 \\
\hline \hline $\mathrm{Ni} K$ & 0.46 & 0.09 & $\mathrm{Ni}$ & 0.46 \\
\hline $\mathrm{Ni} L$ & $\cdots$ & $\cdots$ & & $\cdots$ \\
\hline Total & 100.00 & 100.00 & & 100.00 \\
\hline
\end{tabular}

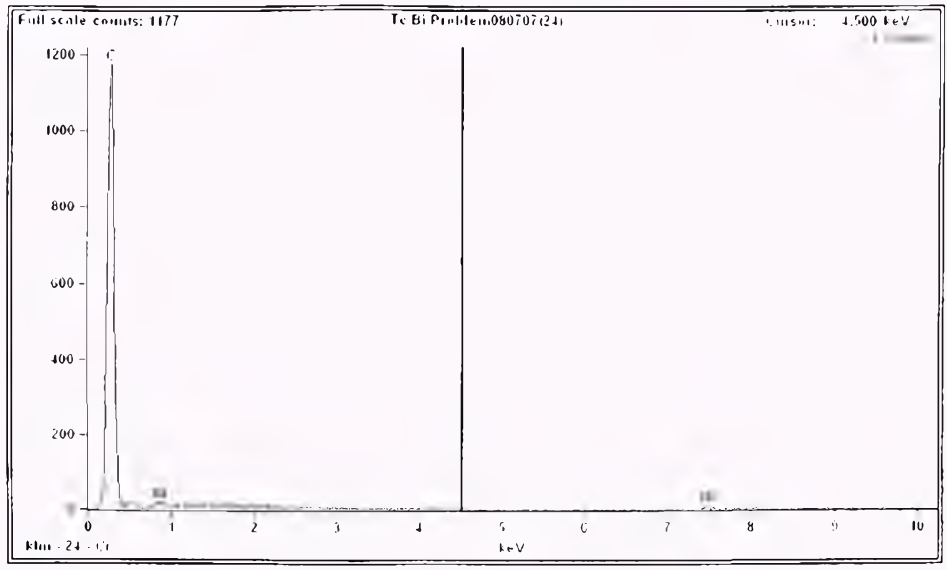

Figure 7.26: EDS analysis of pure CNT's powder sintered at $750^{\circ} \mathrm{C}$ under an inert atmosphere. 


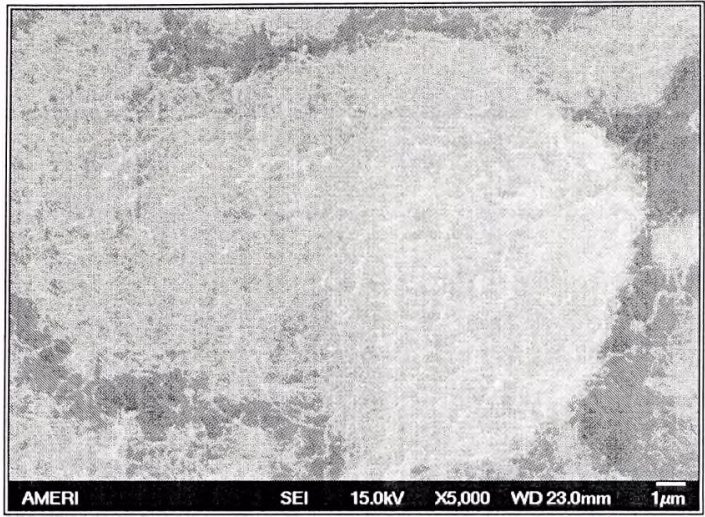

Figure 7.27: SEM image of pure CNTs powder sintered at $900^{\circ} \mathrm{C}$ under an inert atmosphere.

\begin{tabular}{|c||c|c|c|c|}
\hline Element Line & Weight \% & Atom \% & Formula & Compna \% \\
\hline C $K$ & 100.00 & 100.00 & C & 100.00 \\
\hline \hline $\boldsymbol{O} K$ & 0.00 & 0.00 & 0 & 0.00 \\
\hline \hline Total & 100.00 & 100.00 & & 100.00 \\
\hline
\end{tabular}

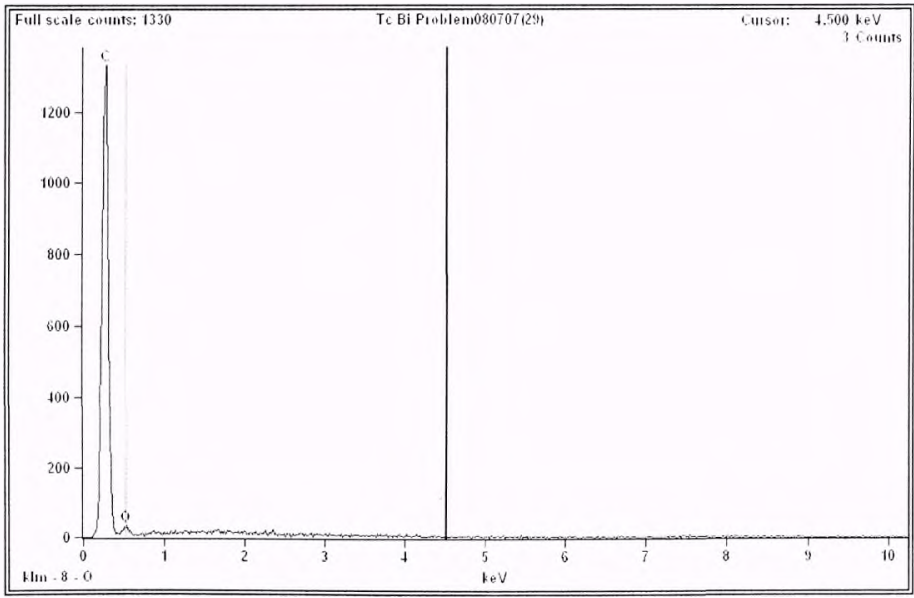

Figure 7.28: EDS analysis of pure CNTs powder sintered at $900{ }^{\circ} \mathrm{C}$ under an inert atmosphere. 


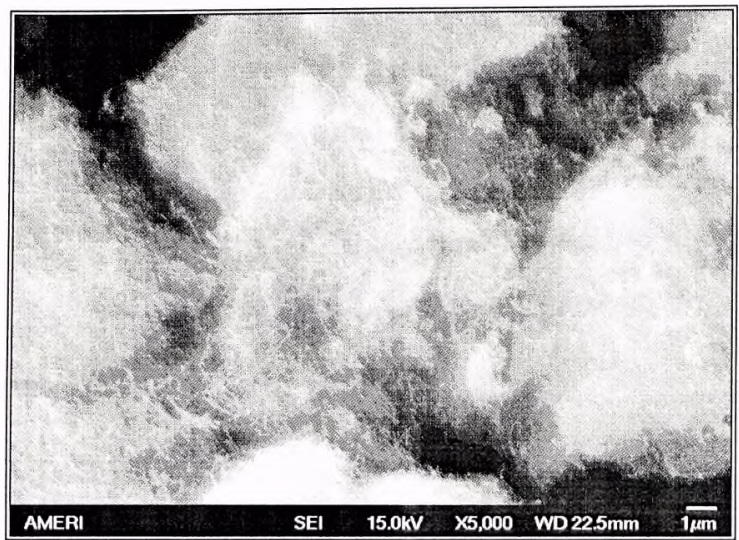

Figure 7.29: SEM image of CNTs sintered at $1050^{\circ} \mathrm{C}$.

\begin{tabular}{|c|c|c|c|c|}
\hline Element Line & Weight \% & Atom \% & Formula & Compnd \% \\
\hline CK & 99.48 & 99.89 & $\bar{C} \mathrm{C}$ & 99.48 \\
\hline$O K$ & 0.00 & 0.00 & $\overline{\mathrm{O}}$ & 0.00 \\
\hline Ni K & 0.52 & 0.11 & $\mathrm{Ni}$ & 0.52 \\
\hline $\mathrm{NiL}$ & $\ldots$ & ב-.. & & ב-.- \\
\hline Total & 100.00 & 100.00 & & 100.00 \\
\hline
\end{tabular}

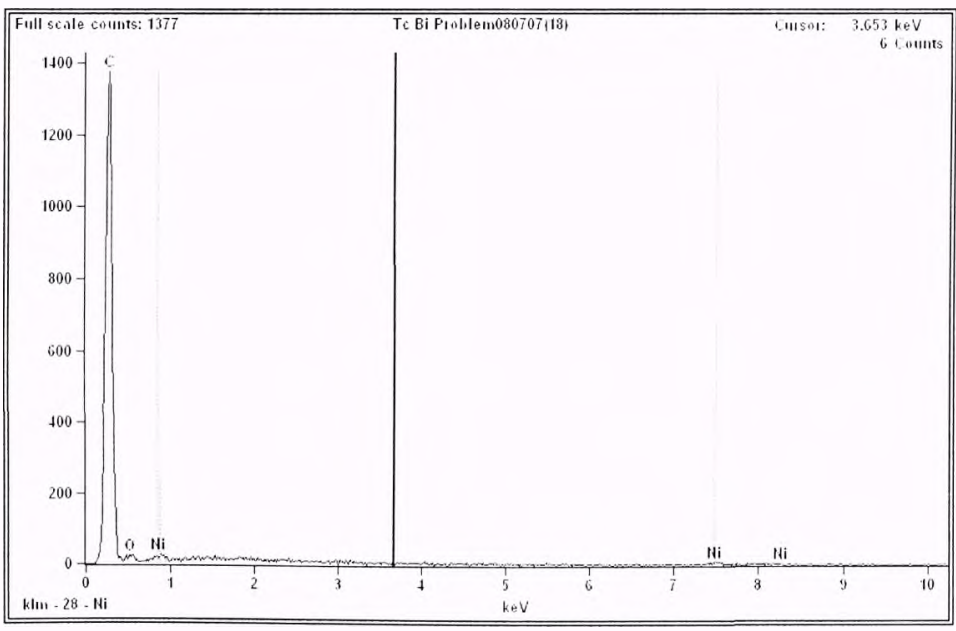

Figure 7.30: EDS analysis of pure CNTs powder sintered at $1050{ }^{\circ} \mathrm{C}$ under an inert atmosphere. 


\section{EXPERIMENTAL RESULTS}

\subsection{Cu-Cr-MWCNT Composite}

The Cu-Cr-MWCNT composite was prepared by using the powder metallurgy methodology. Powder metallurgy is an eflicient and versatile method for manufacturing ferrous and non-ferrous machine parts and electronic components, where unique nonequilibrium compositions and structures are possiblc without melting.

\subsection{Powder Mixing}

Pure powders: $\mathrm{Cu}(99.9 \%, 1-5 \mu-\mathrm{m}), \mathrm{Cr}(99.8 \%, 1-5 \mu-\mathrm{m})$ and MWCNT's $(99 \%$ pure, OD 40-60 nm) were mixed in a glove box, in the proportions shown in Table 8.1 The chambers of the glove box were evacuated and flooded with argon gas to maintain a stable and inert environment (to minimize oxidation since all particles are highly reactive at such small sizes).

Table 8.1: Compositions of $\mathrm{Cu}-\mathrm{Cr}$ alloy and composites.

\begin{tabular}{|c|c|c|c|c|c|c|c|c|}
\hline \multicolumn{3}{|c|}{ Weight in grams } & \multicolumn{3}{c|}{ Weight \% } & \multicolumn{3}{c|}{ Volume \% } \\
\hline Cu & Cr & MWCNT & Cu & Cr & MWCNT & Cu & Cr & MWCNT \\
\hline 3.4 & 0.60 & - & 85 & 15 & - & 82.1849 & 17.9074 & - \\
\hline 3.40 & 0.51 & 0.0888 & 85 & 12.7798 & 2.2202 & 77.6566 & 14.4163 & 8 \\
\hline 3.40 & 0.49 & 0.1126 & 85 & 12.1838 & 2.8162 & 76.5248 & 13.5437 & 10 \\
\hline 3.40 & 0.46 & 0.1372 & 85 & 11.5701 & 3.4299 & 75.3931 & 12.6712 & 12 \\
\hline 3.40 & 0.44 & 0.1625 & 85 & 10.9377 & 4.0623 & 74.2616 & 11.7988 & 14 \\
\hline 3.40 & 0.41 & 0.1886 & 85 & 10.2857 & 4.7143 & 73.1302 & 10.9265 & 16 \\
\hline
\end{tabular}




\subsection{Milling}

High energy ball milling was conducted using a SPEX 8000 mill with a hardened steel vial $(\varphi 21 / 4$ in $\times 3$ in) as the milling container and stainless steel balls as grinding medium, at a fixed milling speed of about $1200 \mathrm{rpm}$. A 10:1 Ball to Powder Ratio (BPR) was used with $\mathrm{Cu}$ and $\mathrm{Cr}$ for 240 minutes using $\varphi \frac{1 / 2}{2}$ inch balls, followed by mixing of Cu-Cr powder with CNT powder for 5 minutes using $\varphi \frac{1 / 4}{4}$ inch balls at a BPR of $3: 1$. CNTs were added in the later stage with $3: 1 \mathrm{BPR}$ because this prevented significant damage to the CNI"s. Various BPR and ball milling times were tried in order to minimize damage to the CNTs, but the optimum BPR and mixing time were $3: 1$ and 5 minutes respectively [3]. The walls of the container were scratched after every 20 minutes in order to remove attached particles. The milled powder was then collected and stored in an airtight bottle within the glove box.

\subsection{Pressing/Pelletizing/Consulidation}

A stainless steel die was used to consolidate milled powders into pellets. In the reviews, it was reported that CNTs fill the void spaces between metal powder particles during compaction leading to an increase in compactness. After the void spaces are filled with CNTs, any further addition lead to a decrease in the degree of compaction (increased porosity). As depicted in Figure 8.1, the compactness of the Cu-CNT composite increased progressively with increasing volume fraction of $\mathrm{CN}$ 'ls and reached a peak value at 12 Vol. \%, then decreased with further addition of CNTs. 


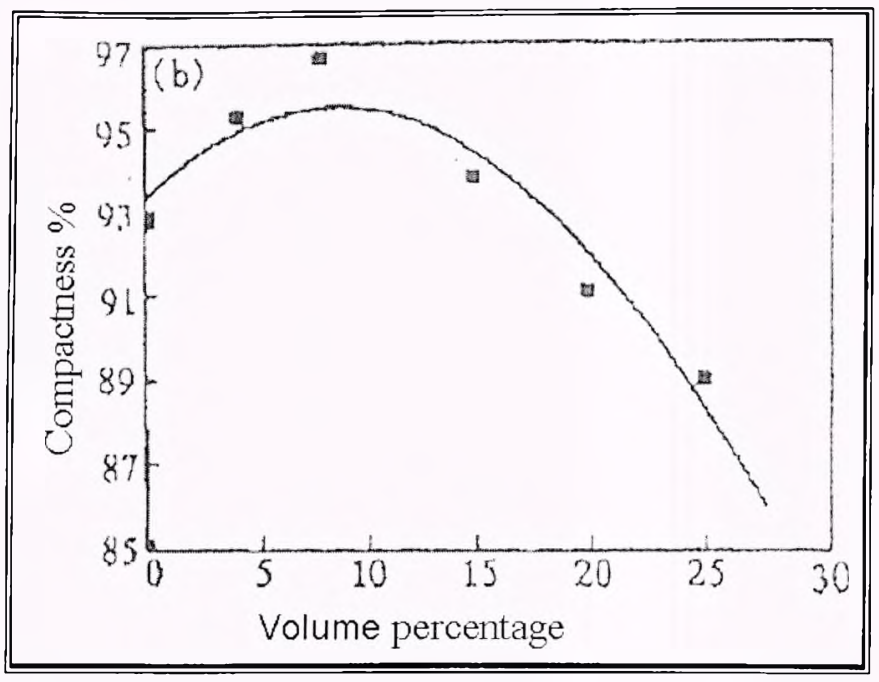

Figure 8.1: Dependency of Compactness of CNTs reinforced C'u based composite on the content of CNTS [31].

In this research the powder was uni-axially cold pressed under vacuum at 844 MPa (25,000 lbs force) using a manual pellet press and held at that pressure for 90 minutes to form the pellet. The pellets were stored in the glove box in separate labeled plastic boxes under inert atmosphere.

\subsection{SEM/EDS Analysis of the Samples Before Sintering}

The morphology of unsintered and sinter composites were investigated using SEM for a comparative analysis. Figure 8.2, shows the SEM photomicrograph of Cu-Cr compact before sintering. There was evidence of pores in the unsintered compacts. some of which disappeared after sintering. 


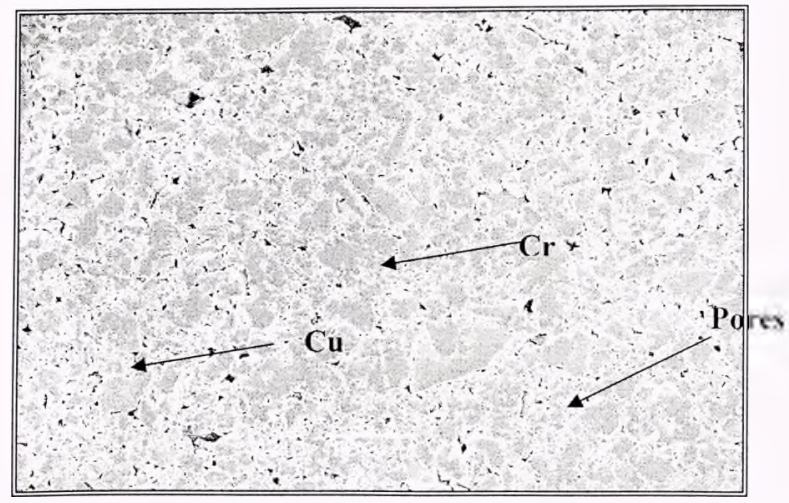

Figure 8.2: SEM analyses for Cu-Cr composite before sintering.

Backscattered image of the Cu-Cralloy was taken to determine the chemistry of the composite. It was observed that the darker region was indicative of $\mathrm{Cr}$ (At. wt. 52) where as Cu (At. wt. 63.55), possessing a greater atomic weight, has less absorption and more reflection. The photomicrograph of Figure 8.3 revealed a homogenous bi-model morphology.

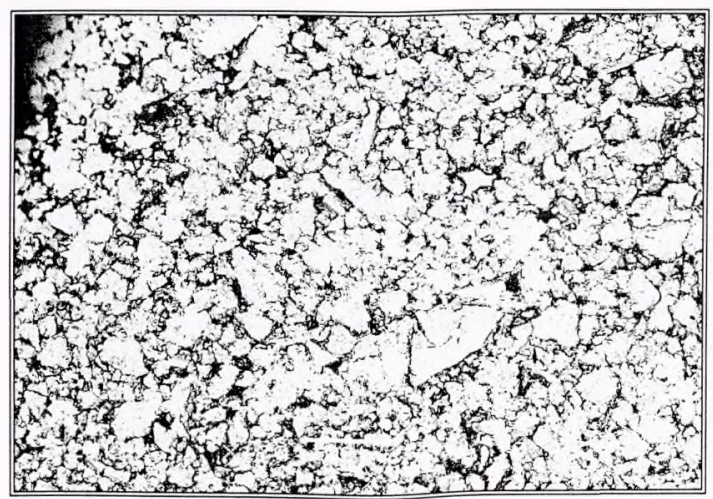

Figure 8.3: Backscatter image of Cu-Cr alloy. 


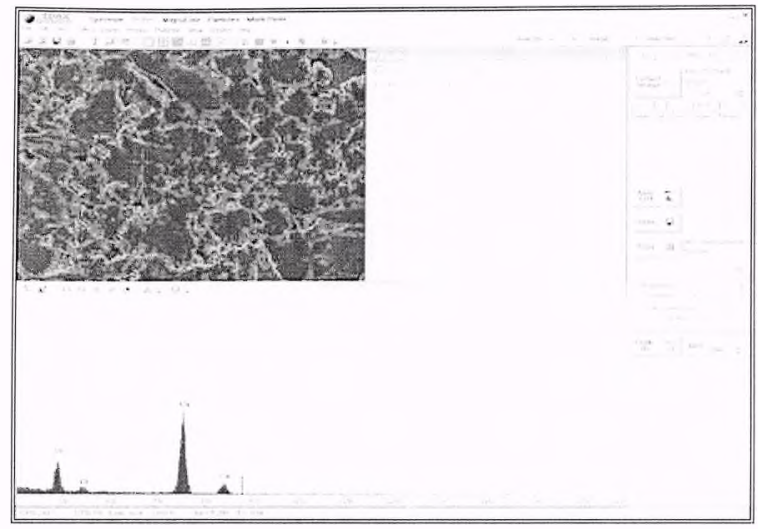

Figure 8.4: EDS analysis for $\mathrm{Cu}-\mathrm{Cr}$ alloy

\subsubsection{SEM and EDS Analysis}

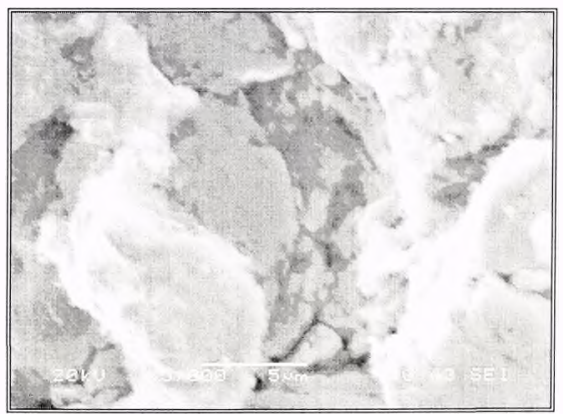

Figure 8.5: SEM image of Cu-Cr 8 Vol \% MWCNT composite. 


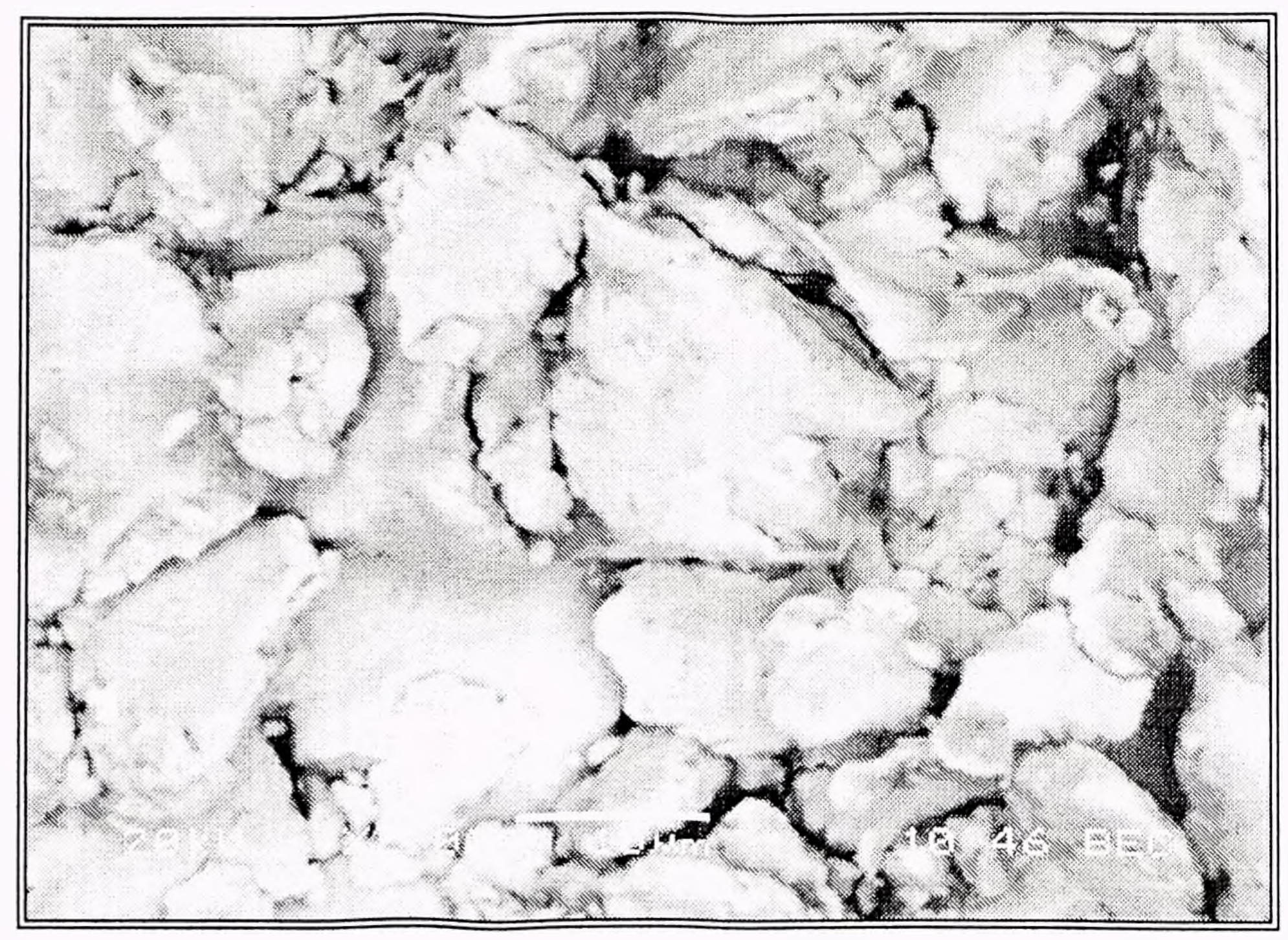

Figure 8.6: Backscatter image of $\mathrm{Cu}-\mathrm{Cr} 8 \mathrm{Vol}$. \% MWCNT composite.

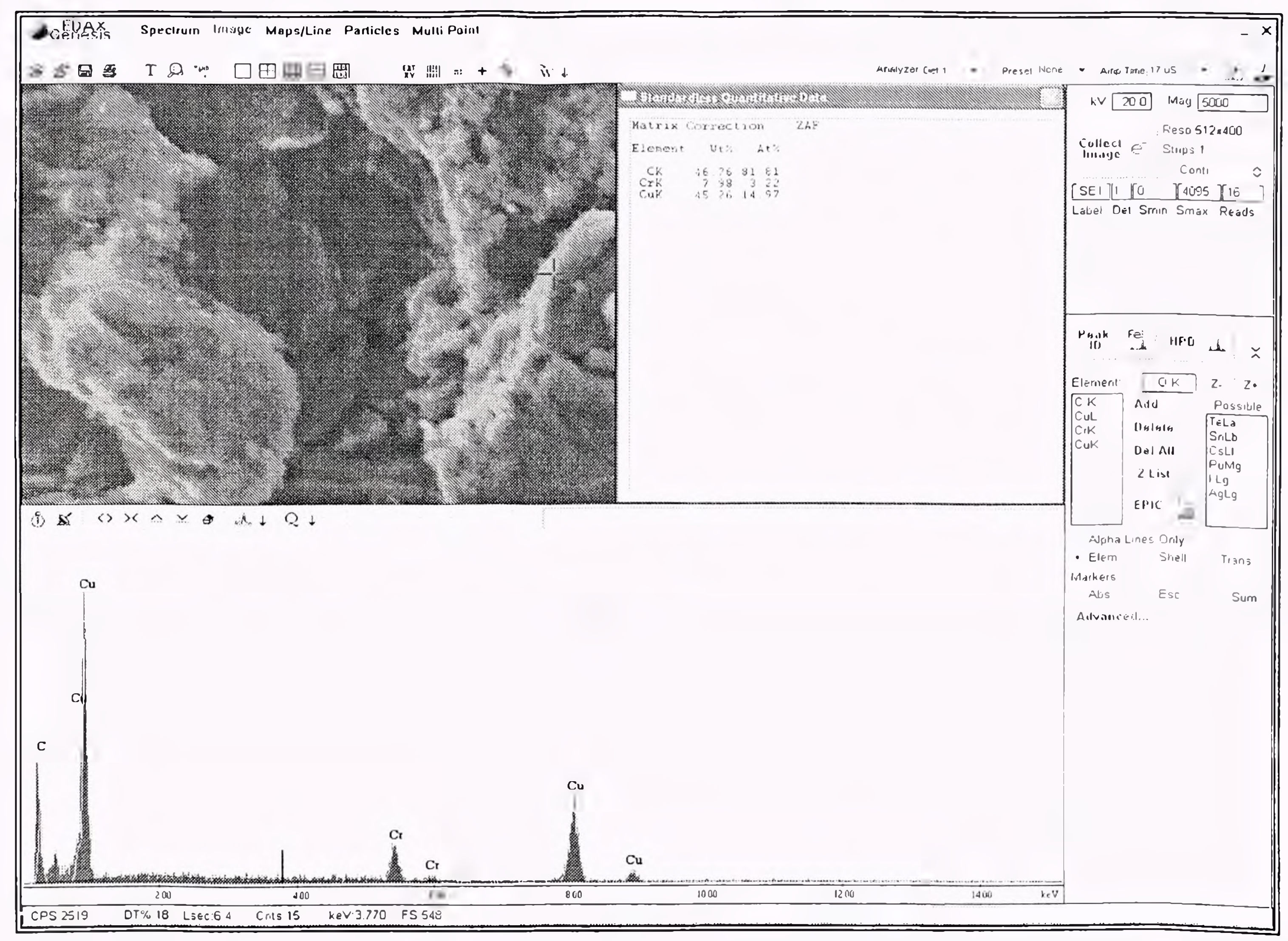

Figure 8.7: EDS analysis of $\mathrm{Cu}-\mathrm{Cr} 8 \mathrm{Vol}$ \% MWCNT composite. 


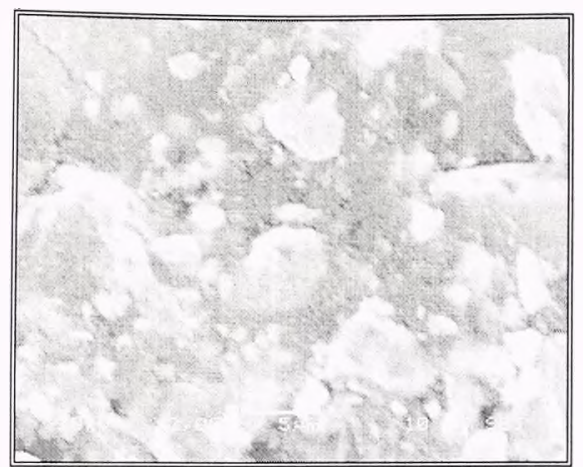

Figure 8.8: SEM image of Cu-Cr $10 \mathrm{Vol}$. $\%$ MWCNT

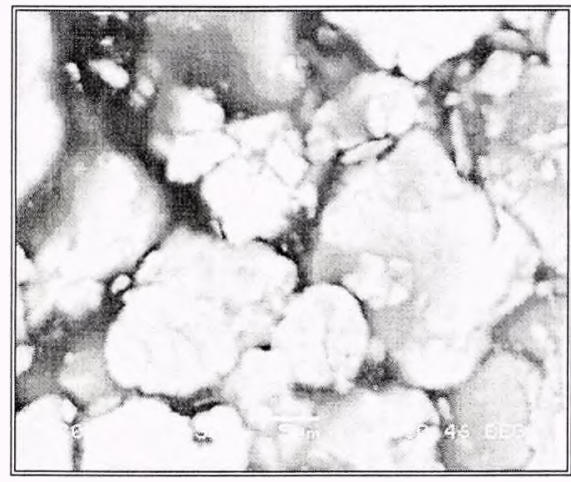

Figure 8.10: Backscatter image of $\mathrm{Cu}-\mathrm{Cr}$ 14 Vol. \% MWCNT.

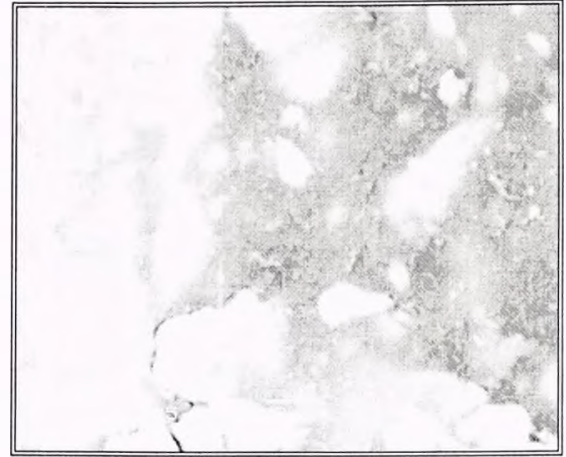

Figure 8.9: SEM image of Cu- Cr 12 Vol. \% MWCNT

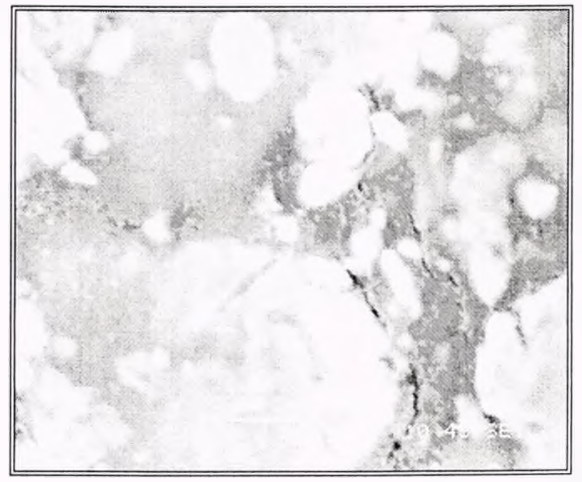

Figure 8.11: Backscatter image of $\mathrm{Cu}-\mathrm{Cr} 16$ Vol. \% MWCNT.

\subsection{Sintering}

The composites were sintered in a Lindberg/Blue tube furnace at three different temperatures i.e. $750{ }^{\circ} \mathrm{C}, 900^{\circ} \mathrm{C}$ and $1050^{\circ} \mathrm{C}$. In order to avoid oxidation, sintering was performed under argon (99.9999\%), utilizing a ramp time of 1 hour and a hold time of 1.5 hours. Compacts were allowed to cool down slowly to room temperature. 
The sintering temperature was determined by using the following equation. The CNTs was not included in the formula because of its very high melting point.

Sintering Temperature $=[(\mathrm{Mol}$ fraction $\mathrm{Cu}) \times(\mathrm{Cu} T \mathrm{~m})+(\mathrm{Mol}$ fraction $\mathrm{Cr}) \times(\mathrm{Cr}$ $[\mathrm{m})]^{*}(2 / 3)$

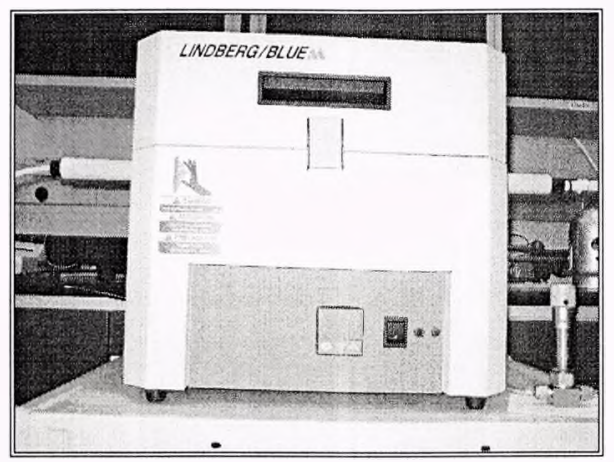

Figure 8.12: Lindberg/Blue tube furnace, with quartz tube flooded with argon.

\subsection{Electrical Conductivity}

The electrical resistivity of the sintered compacts was measured using a four probe HP 4263A LCR meter. The resistivity was calculated using the formula:

$$
\rho=(\pi I / \ln 2) V / I \text { for } s>>1
$$

where, $\rho$ is resistivity, $\mathrm{R}=\mathrm{V} / \mathrm{I}$ is resistance. $\mathrm{s}$ is the distance between the probes and $t$ is the thickness of the sample. The electrical conductivity of the compact was defined as the reciprocal of the electrical resistivity.

The results of the electrical conductivity of composites with varying MWCNT content Vs temperature at NTP are shown in Figure 8.13 and the data are summarized in Table 8.2 
Table 8.2: Electrical conductivity at $290 \mathrm{~K}$ of composites, sintered at $750^{\circ} \mathrm{C}$.

\begin{tabular}{|c|c|}
\hline Sample (sintered, $750^{\circ} \mathrm{C}$ ) & Electrical conductivity at $290 \mathrm{~K} .(\mathrm{S} / \mathrm{m})$ \\
\hline $\mathrm{Cu}-\mathrm{Cr} 15 \mathrm{wt} . \%$ & $3.44 \mathrm{E}+07$ \\
\hline Cu-Cr 8 Vol.\% MWCNT & $2.50 \mathrm{E}+07$ \\
\hline Cu-Cr 10 Vol.\% MWCNT & $7.38 \mathrm{E}+08$ \\
\hline Cu-Cr 12 Vol.\% MWCNT & $4.43 E+08$ \\
\hline $\mathrm{Cu}-\mathrm{Cr} 14 \mathrm{Vol} . \% \mathrm{MWCNT}$ & $1.96 \mathrm{E}+08$ \\
\hline Cu-Cr 16 Vol.\% MWCNT & $8.61 \mathrm{E}+07$ \\
\hline
\end{tabular}

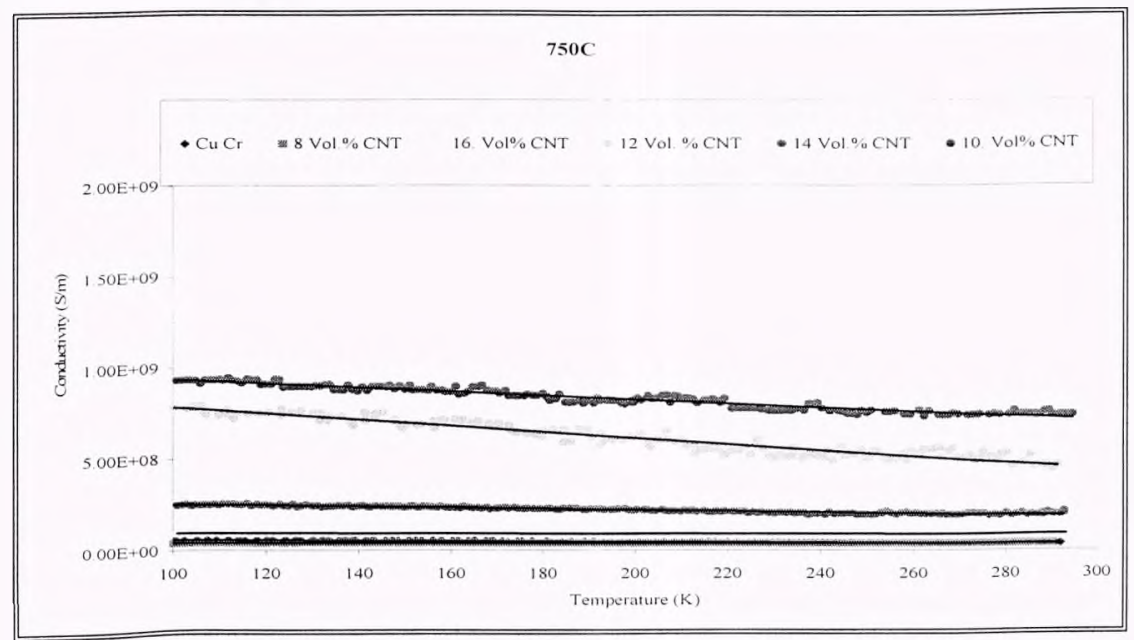

Figure 8.13: Plot of Electrical conductivity Vs Temperature for samples sintered at $750^{\circ} \mathrm{C}$.

Table 8.3: Electrical conductivity at $290 \mathrm{~K}$ of composites. sintered at $900^{\circ} \mathrm{C}$.

\begin{tabular}{||c|c||}
\hline \hline Sample (sintered, $\left.900{ }^{\circ} \mathrm{C}\right)$ & Electrical conductivity at 290 K. (S/m) \\
\hline $\mathrm{Cu}-\mathrm{Cr} 15$ wt.\% & $3.87 \mathrm{E}+07$ \\
\hline $\mathrm{Cu}-\mathrm{Cr} 8$ Vol.\% MWCNT & $4.44 \mathrm{E}+07$ \\
\hline $\mathrm{Cu}-\mathrm{Cr} 10 \mathrm{Vol} . \% \mathrm{MWCNT}$ & $1.11 \mathrm{E}+09$ \\
\hline $\mathrm{Cu}-\mathrm{Cr} 12 \mathrm{Vol} . \% \mathrm{MWCNT}$ & $0.20 \mathrm{E}+08$ \\
\hline $\mathrm{Cu}-\mathrm{Cr} 14 \mathrm{Vol} . \% \mathrm{MWCNT}$ & $4.06 \mathrm{E}+08$ \\
\hline $\mathrm{Cu}-\mathrm{Cr} 16 \mathrm{Vol} . \% \mathrm{MWCNT}$ & $1.58 \mathrm{E}+08$ \\
\hline
\end{tabular}




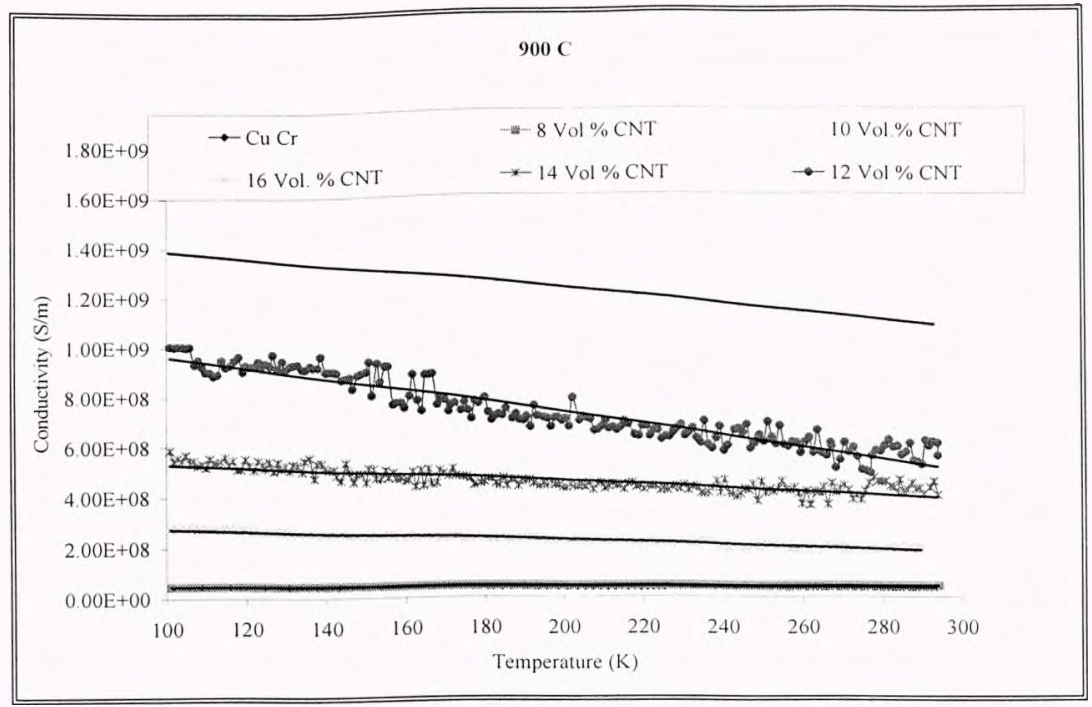

Figure 8.14: Plot for Electrical conductivity Vs Temperature for composites sintered at $900{ }^{\circ} \mathrm{C}$.

\subsection{Density \& Porosity Measurement}

The apparent porosity was estimated from the amount of water absorbed by a given weight or volume of the composite by using the following formula

$$
P=\frac{W_{2}-W_{1}}{\left(W_{2}-W_{1}\right) \rho}
$$

Where, $W_{1}$ is weight of the thoroughly dried composite. $W_{2}$ is the weight of the saturated sample weighed in air, $\mathrm{W}_{3}$ is the weight of the composite when immersed in water, and $\rho$ is the density of the liquid which will be $1 \mathrm{~g} / \mathrm{cc}$ if water is used. 
Table 8.4: Variation of porosity with MWCNT volume percentage at three sintering temperatures

\begin{tabular}{||c|c|c|c|}
\hline \multirow{2}{*}{ Sample } & \multicolumn{3}{|c|}{ Sintering Temperature } \\
\cline { 2 - 4 } & $\mathbf{7 5 0} \mathbf{~}^{\circ} \mathbf{C}$ & $\mathbf{9 0 0}{ }^{\circ} \mathbf{C}$ & $\mathbf{1 0 5 0}{ }^{\circ} \mathbf{C}$ \\
\hline Cu-Cr 8 Vol.\% MWCNT & 0.1784 & 0.1299 & 0.1682 \\
\hline Cu-Cr 10 Vol.\% MWCNT & 0.1420 & 0.1203 & 0.1352 \\
\hline Cu-Cr 12 Vol.\% MWCNT & 0.1915 & 0.1497 & 0.1797 \\
\hline Cu-Cr 14 Vol.\% MWCNT & 0.1978 & 0.1793 & 0.1830 \\
\hline Cu-Cr 16 Vol.\% MWCNT & 0.2203 & 0.1878 & 0.2010 \\
\hline
\end{tabular}

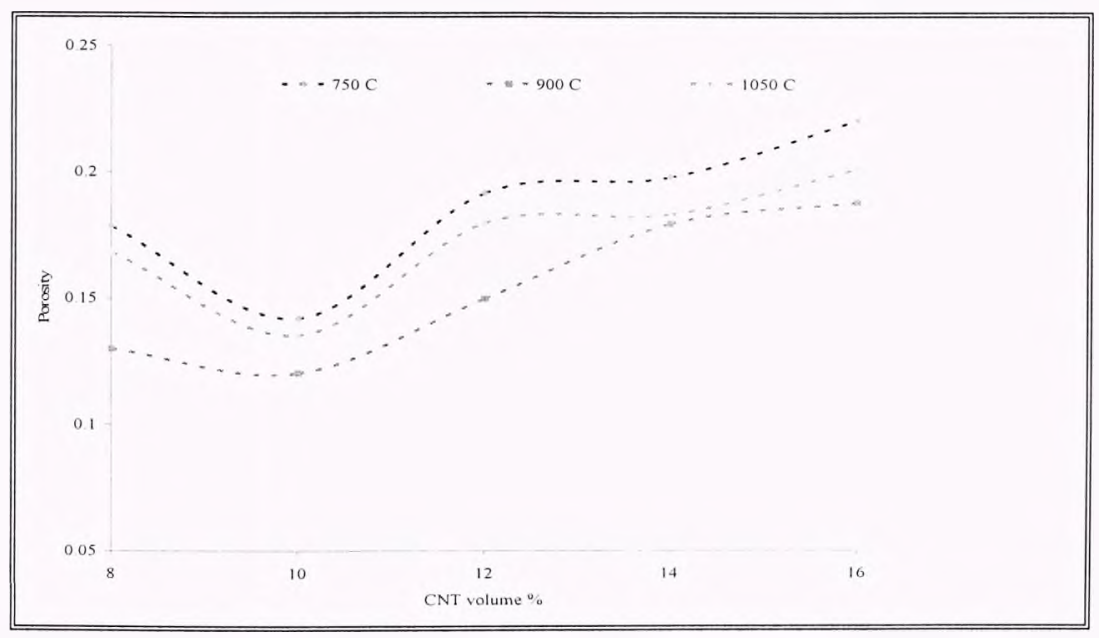

Figure 8.15: Variation of porosity with MWCNT volume percentage at three different sintering temperatures.

\subsection{Vickers Hardness}

Vicker's indentation was used for measuring indentation toughness with a load of $200 \mathrm{~g}$ and a dwell time of $15 \mathrm{~s}$. Shurong and Xiaobin (1999), stated that CNTs have a significant effect on the Vicker's hardness and the compactness of the Cu-CNT 
composite [31]. In their work it was found that the Vicker's hardness increased with increasing volume fraction of CNTs until $12 \mathrm{Vol} . \%$, after which it decreased [31].

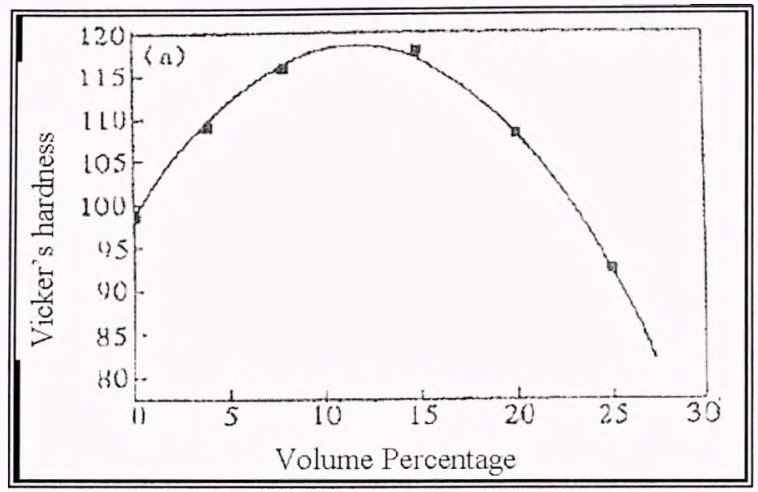

Figure 8.16: Dependency of Compactness of CNTs reinforced Cu based composite on the content of CNTs $|31|$.

The variation of Vickers's hardness was due to fiber strengthening effect and the variation in the degree of compactness with an increase in volume fraction of MWCNT. The Vicker's hardness of composites that were sintered at three temperatures is shown below.

Table 8.5: Vicker's hardness of Cu-Cr MWCNT composites

\begin{tabular}{|c|c|c|c|}
\hline \multirow[b]{2}{*}{ Sample } & \multicolumn{3}{|c|}{ Sintering Temperature } \\
\hline & $750{ }^{\circ} \mathrm{C}$ & $900{ }^{\circ} \mathrm{C}$ & $1050^{\circ} \mathrm{C}$ \\
\hline $\mathrm{Cu}-\mathrm{Cr} 8 \mathrm{Vol} . \% \mathrm{MWCNT}$ & 101.31 & 119.3 & 131.1 \\
\hline $\mathrm{Cu}-\mathrm{Cr} 10 \mathrm{Vol} . \% \mathrm{MWCNT}$ & 111.9 & 145.5 & 128.1 \\
\hline $\mathrm{Cu}-\mathrm{Cr} 12 \mathrm{Vol} . \% \mathrm{MWCNT}$ & 106.3 & 135.9 & 114.9 \\
\hline Cu-Cr 14 Vol.\% MWCNT & 98.8 & 121.5 & 102.7 \\
\hline Cu-Cr 16 Vol. $\%$ MWCNT & 81.45 & 99.6 & 93.5 \\
\hline
\end{tabular}




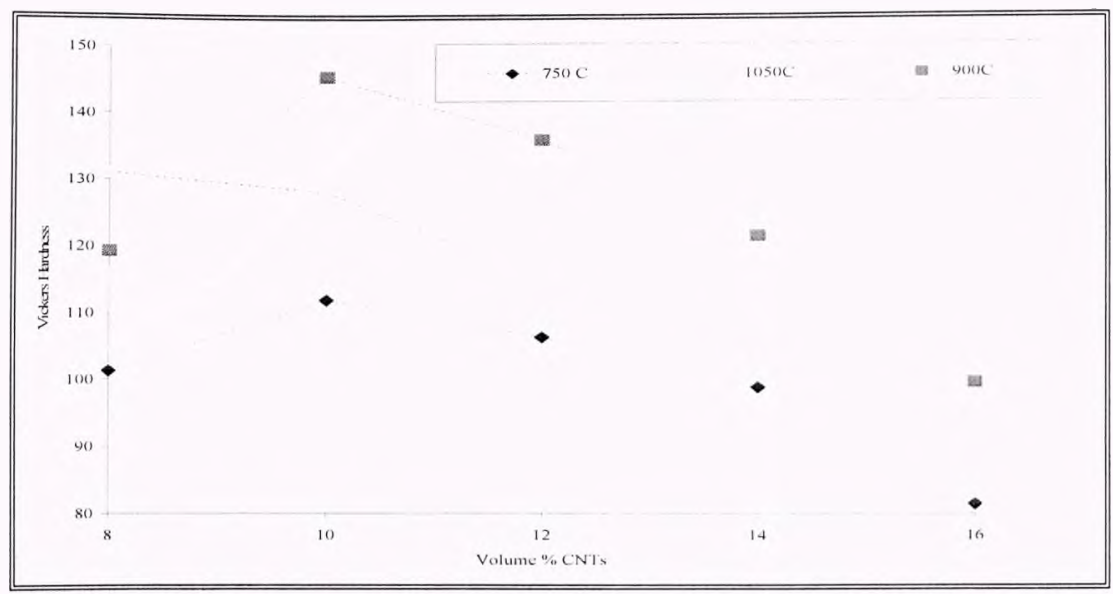

Figure 8.17: Variation of Vicker's hardness of composites with MWCNT content.

\subsection{Raman Spectroscopy}

Raman spectroscopy with a high-resolution (maximum $\sim 50 \mathrm{~cm}^{-1}$ ) f/1.8 double monochromator was used to investigate the extent of any destruction of CNTs in the composite during milling, compaction and after sintering. A comparison of the ratios of D-line and G-line provides a measure of the quality of the CNT within the composite. The PM dark current was $13 \mathrm{M}$ watts and laser excitation, $514 \mathrm{~nm}$. Table 8.6 shows the position and full-width at half maximum (FWHM) of the D and (is spectra for various compositions of composites before sintering.

The high sintering temperature, ball milling and consolidation of the compact resulted in some damage of the MWCNTs. Thus initial Raman spectrum data assisted us in realizing the importance of optimized working parameters in retaining CNTs. Raman spectroscopy was used to evaluate the effect of sintering temperature (i.e. $750^{\circ} \mathrm{C}, 900^{\circ} \mathrm{C}$ and $1050{ }^{\circ} \mathrm{C}$ ) on the MWCNTs. 
This was accomplished by comparing the ratio, R. between the $D$ ) and $G$ bands $\left(\mathrm{I}_{\mathrm{D}} / \mathrm{l}_{\mathrm{G}}\right)$ of the as received MWCNT before and after sintering as well as in composites before and after sintering. A ratio of $\sim 0.92$ (unsintered MWCNTs) was compared with $0.89-1.11$ for unsintered composites.

Owing to inhomogenieties in the size of the tubes, varying defect density, agglomeration, compositions and rough sample surface, values between $1344-1360 \mathrm{~cm}^{-1}$ were observed for the D band and $1572-1587 \mathrm{~cm}^{-1}$ were observed for the G band. The D peak shift $\sim-10.01-6.25$, and a G peak shift of $\sim-8.2-5.84$ was observed with respect to pure unsintered MWCNT.

Table 8.6: Comparison of the spectral parameters in the Raman spectra before sintering.

\begin{tabular}{|c|c|c|c|c|c|}
\hline \multirow{2}{*}{ Unsintered Sample } & \multicolumn{2}{|c|}{ D band $\left(\mathrm{cm}^{\prime}\right)$} & \multicolumn{2}{|c|}{ G band $\left(\mathrm{cm}^{-1}\right)$} & \multirow{2}{*}{$\begin{array}{c}\text { Ratio }= \\
\mathbf{I}_{D} / \mathbf{I}_{i}\end{array}$} \\
\hline & Position & FWHM & Position & FWHM & \\
\hline Pure MW'CNT (As-received) & 1354.52 & 61.35 & 1580.73 & +8.1 & 0.92 \\
\hline Cu-Cr 8 Vol.\% MWCNT & 1360.77 & 44.32 & 1586.57 & 32.75 & 1.11 \\
\hline $\mathrm{Cu}-\mathrm{Cr} 10 \mathrm{Vol} . \% \mathrm{MWCNT}$ & 1355.77 & 36.22 & 1586.57 & 37.31 & 1.04 \\
\hline $\mathrm{Cu}-\mathrm{Cr} 12 \mathrm{Vol} . \% \mathrm{MWCNT}$ & 1344.51 & 51.38 & 1580.75 & 47.51 & 0.97 \\
\hline $\mathrm{Cu}-\mathrm{Cr} 14 \mathrm{Vol} . \% \mathrm{MWCNT}$ & 1357.02 & 52.1 & 1586.57 & 39.67 & 0.91 \\
\hline $\mathrm{Cu}-\mathrm{Cr} 16 \mathrm{Vol} . \% \mathrm{MWCNT}$ & 1347.01 & 38.41 & 1572.53 & 53.65 & 0.90 \\
\hline
\end{tabular}

The positions of the $D$ and $G$ bands are summarized in Table 8.6. with the objective to determine the peak shift, which is another indicator of strained MW'CNTs in the composites. 


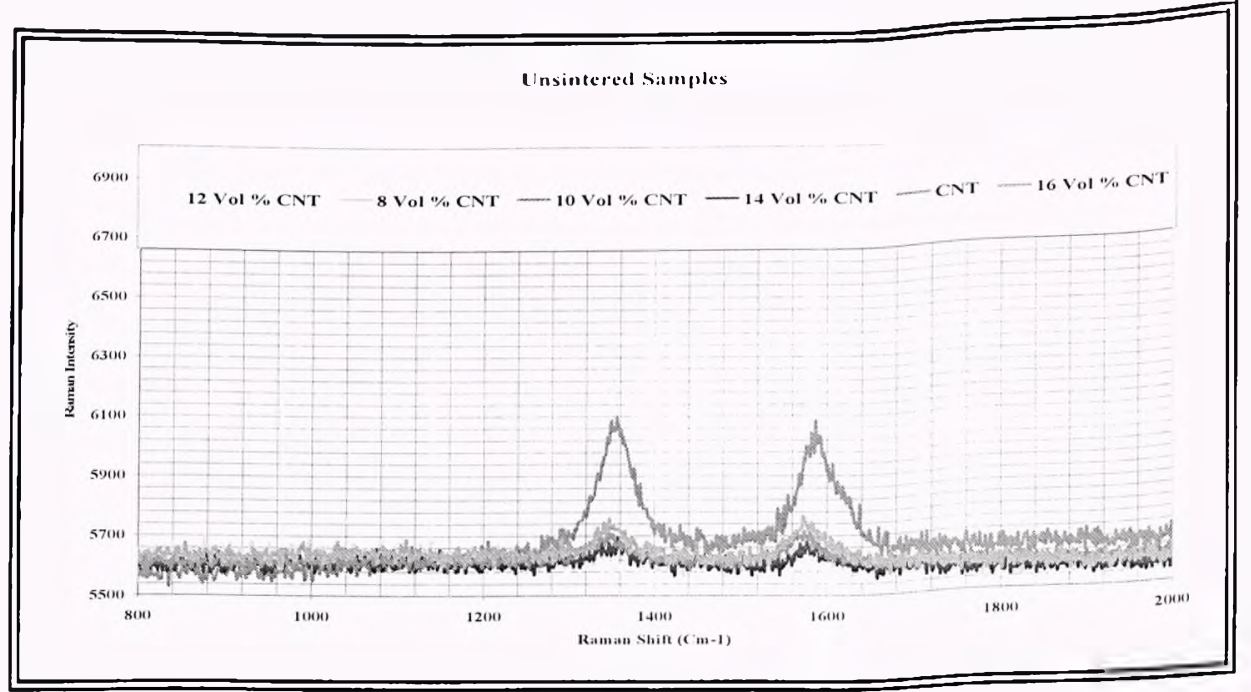

Figure 8.18: Plot for unsintered samples.

MWCNTs were sintered at three different sintering temperatures under inert atmosphere conditions with 1.5 hours of hold time. The MWCNTs sintered at $750{ }^{\circ} \mathrm{C}$. had $\mathrm{R}$ values ranging between $0.74-1.14$. The $\mathrm{D}$ peak shift ranged between $-8.76-6.25$. and the G peak shift ranged between $-9.37-7.01$ with respect to MWCNT.

Table 8.7: Comparison of the spectral parameters in the Raman spectra of samples sintered at $7500^{\circ} \mathrm{C}$.

\begin{tabular}{|c|c|c|c|c|c|}
\hline \multirow[b]{2}{*}{ Sintered $\left(750^{\circ} \mathrm{C}\right)$} & \multicolumn{2}{|c|}{ D band } & \multicolumn{2}{|c|}{ G band } & \multirow[b]{2}{*}{$\begin{array}{c}\text { Ratio } \\
\left.\mathrm{R}=\mathrm{I}_{1}\right) / \mathrm{I}_{(;}\end{array}$} \\
\hline & $\begin{array}{c}\text { Position } \\
\left(\mathrm{cm}^{-1}\right)\end{array}$ & $\begin{array}{c}\text { FWIIM } \\
\left(\mathrm{cm}^{-1}\right)\end{array}$ & $\begin{array}{c}\text { Position } \\
\left(\mathrm{cm}^{-1}\right)\end{array}$ & $\begin{array}{c}\text { FWHM } \\
\left(\mathrm{cm}^{\mathrm{I}}\right)\end{array}$ & \\
\hline Pure MWCNT (sintered) & 1345.76 & 50.8 & 1571.36 & 41.04 & 0.74 \\
\hline $\mathrm{Cu}-\mathrm{Cr} 8 \mathrm{Vol} . \% \mathrm{MWCNT}$ & 1357.02 & 61.14 & 1586.57 & 38.51 & 0.88 \\
\hline $\mathrm{Cu}-\mathrm{Cr} 10 \mathrm{Vol} . \% \mathrm{MWCNT}$ & 1360.76 & 42.48 & 1587.74 & 31.51 & 0.88 \\
\hline Cu-Cr 12 Vol.\% MWCNT & 1355.77 & 37.53 & 1580.73 & 47.88 & 1.03 \\
\hline $\mathrm{Cu}-\mathrm{Cr} 14 \mathrm{Vol} . \% \mathrm{MWCNT}$ & 1360.77 & 46.24 & 1587.74 & 32.68 & 1.14 \\
\hline $\mathrm{Cu}-\mathrm{Cr} 16 \mathrm{Vol} \% \mathrm{MWCNT}$ & 1355.77 & 41.24 & 1586.57 & 39.61 & 1.14 \\
\hline
\end{tabular}




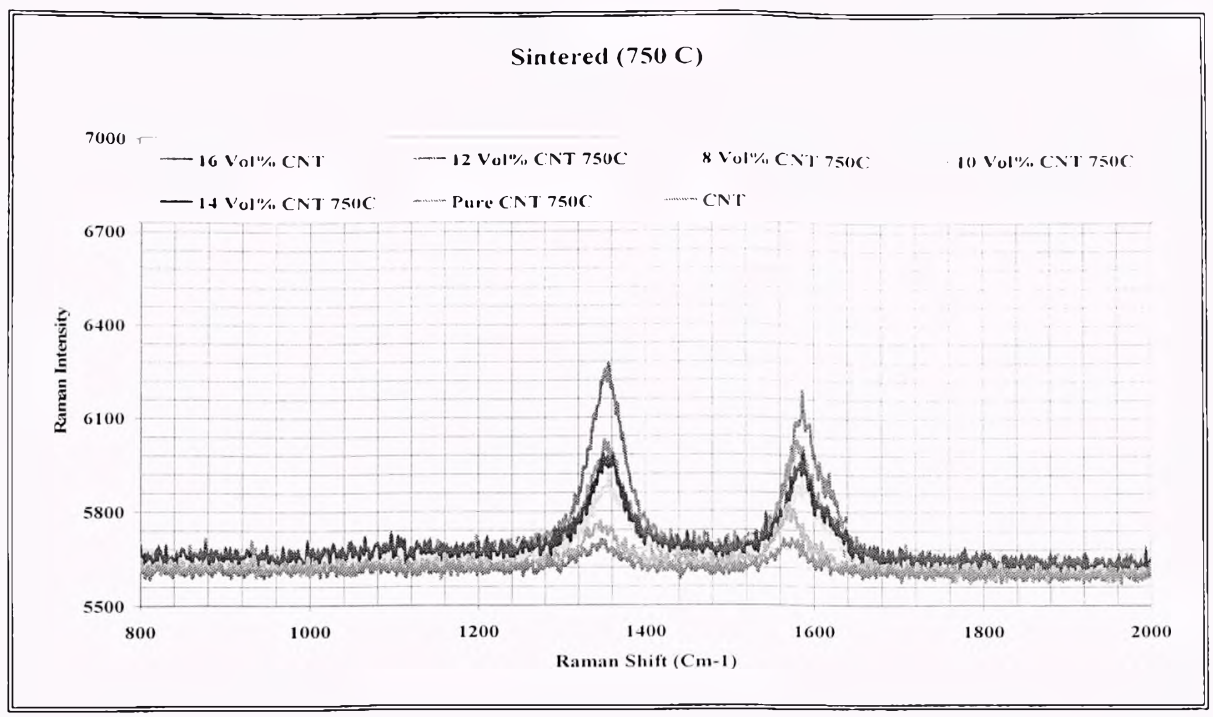

Figure 8.19: Raman Spectra of sintered samples at $750^{\circ} \mathrm{C}$.

The composites sintered at $900{ }^{\circ} \mathrm{C}$ had an $\mathrm{R}$ value of $\sim 0.73$. The $\mathrm{D}$ peak shift ranged between $-17.54-1.25$, and the G peak shift ranged between $-9.37-8.17$ with respect to MWCNT.

Table 8.8: Comparison of the spectral parameters in the Raman spectra of composites sintered at $900^{\circ} \mathrm{C}$.

\begin{tabular}{|c|c|c|c|c|c|}
\hline \multirow[b]{2}{*}{ Sintered $\left(900^{\circ} \mathrm{C}\right)$} & \multicolumn{2}{|c|}{ D band } & \multicolumn{2}{|c|}{ G band } & \multirow[b]{2}{*}{$\begin{array}{l}\text { Ratio } \\
\left.\mathrm{R}=\mathrm{I}_{1}\right) / \mathbf{I}_{\mathrm{G}}\end{array}$} \\
\hline & $\begin{array}{c}\text { Position } \\
\left(\mathrm{cm}^{1}\right)\end{array}$ & $\begin{array}{c}\text { FWHM } \\
\left(\mathrm{cm}^{-1}\right)\end{array}$ & $\begin{array}{l}\text { Position } \\
\text { (cm ') }\end{array}$ & $\begin{array}{l}\text { FWHM } \\
\left(\mathrm{cm}^{1}\right)\end{array}$ & \\
\hline Pure MWCNT (sintered) & 1348.27 & 56.33 & 1574.88 & 39.81 & 0.73 \\
\hline Cu-Cr 8 Vol.\% MWCNT & 1349.52 & 52.48 & 1588.9 & 71.61 & 1.14 \\
\hline $\mathrm{Cu}-\mathrm{Cr} 10 \mathrm{Vol} . \% \mathrm{MWCNT}$ & 1336.98 & 56.09 & 1577.22 & 43.32 & 0.92 \\
\hline Cu-Cr 12 Vol.\% MWCNT & 1339.49 & 47.51 & 1571.36 & 40.88 & 1.04 \\
\hline $\mathrm{Cu}-\mathrm{Cr} 14 \mathrm{Vol} . \% \mathrm{MWCNT}$ & 1355.77 & 41.26 & 1586.57 & 34.29 & 1.07 \\
\hline $\mathrm{Cu}-\mathrm{Cr} 16 \mathrm{Vol} . \% \mathrm{MWCNT}$ & 1353.27 & 41.3 & 1586.57 & 53.63 & 1.25 \\
\hline
\end{tabular}




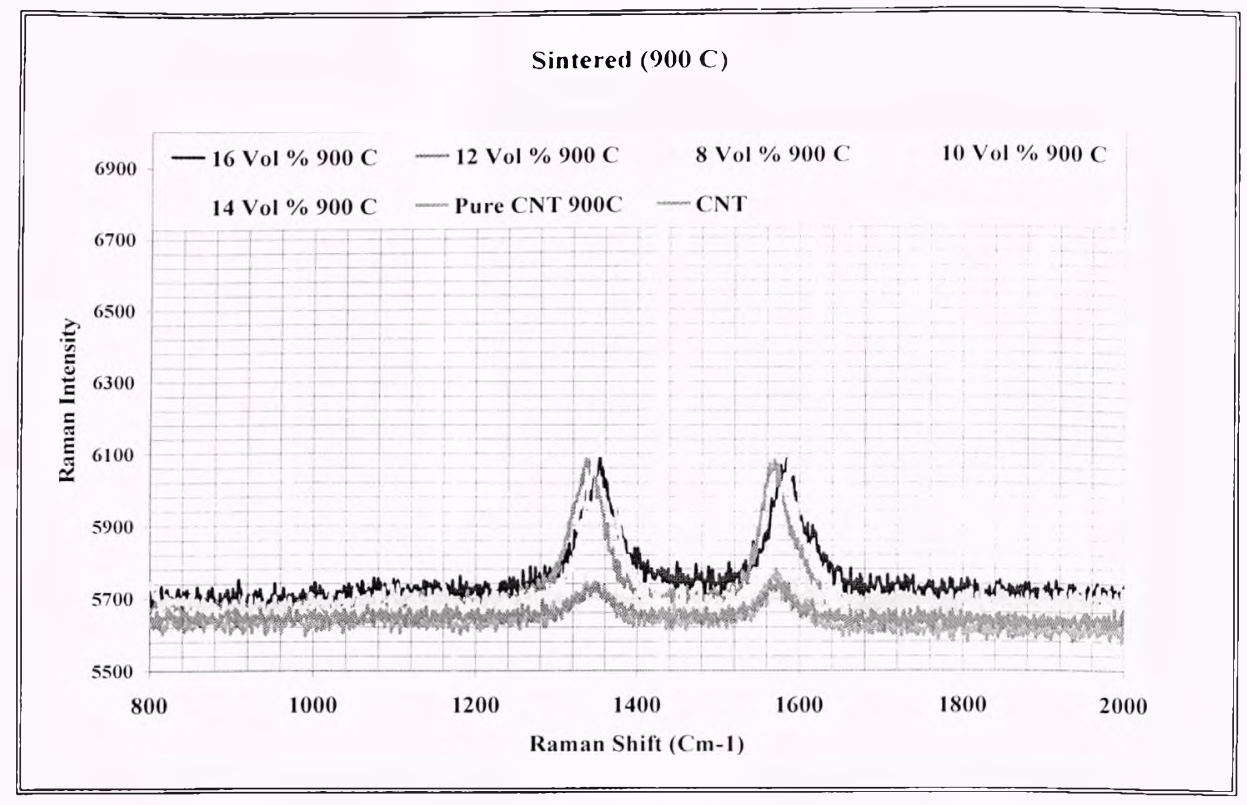

Figure 8.20: Raman spectra of composites sintered at $900^{\circ} \mathrm{C}$.

The composites sintered at $1050{ }^{\circ} \mathrm{C}$ had $\mathrm{R}$ values in the range of $0.71-1.15$. The D peak shift ranged between $-15.03-6.25$, and the G peak shift ranged between - 14.07 12.83 with respect to MWCNT.

Table 8.9: Comparison of the spectral parameters in the Raman spectra of composites sintered at $1050^{\circ} \mathrm{C}$.

\begin{tabular}{|c|c|c|c|c|c|}
\hline \multirow[b]{2}{*}{ Sintered $\left(1050^{\circ} \mathrm{C}\right)$} & \multicolumn{2}{|c|}{ D band } & \multicolumn{2}{|c|}{ G band } & \multirow[b]{2}{*}{$\begin{array}{c}\text { Ratio } \\
\mathrm{R}=\mathrm{I}_{\mathrm{D}} / \mathbf{I}_{\mathrm{G}}\end{array}$} \\
\hline & $\begin{array}{c}\text { Position } \\
\left(\mathrm{cm}^{-1}\right)\end{array}$ & $\begin{array}{c}\text { FWHM } \\
\left(\mathrm{cm}^{1}\right)\end{array}$ & $\begin{array}{c}\text { Position } \\
\left(\mathrm{cm}^{1}\right)\end{array}$ & $\begin{array}{c}\text { FWHM } \\
\left(\mathrm{cm}^{\mathrm{I}}\right)\end{array}$ & \\
\hline Pure MWCNT (sintered) & 1339.5 & 37.56 & 1566.66 & 41.36 & 0.71 \\
\hline Cu-Cr 8 Vol.\% MWCNT & 1342 & 49.17 & 1586.57 & 35.12 & 1.15 \\
\hline $\mathrm{Cu}-\mathrm{Cr} 10 \mathrm{Vol} . \% \mathrm{MWCNT}$ & 1360.8 & 48.64 & 1593.56 & 61.51 & 0.74 \\
\hline $\mathrm{Cu}-\mathrm{Cr} 12 \mathrm{Vol} . \% \mathrm{MWCNT}$ & 1354.5 & 43.78 & 1586.57 & 47.88 & 0.93 \\
\hline $\mathrm{Cu}-\mathrm{Cr} 14 \mathrm{Vol} . \% \mathrm{MWCNT}$ & 1357.02 & 53.69 & 1590.07 & 44.27 & 1.03 \\
\hline Cu-Cr 16 Vol.\% MWCNT & 1360.8 & 41.23 & 1590.07 & 33.74 & 0.98 \\
\hline
\end{tabular}




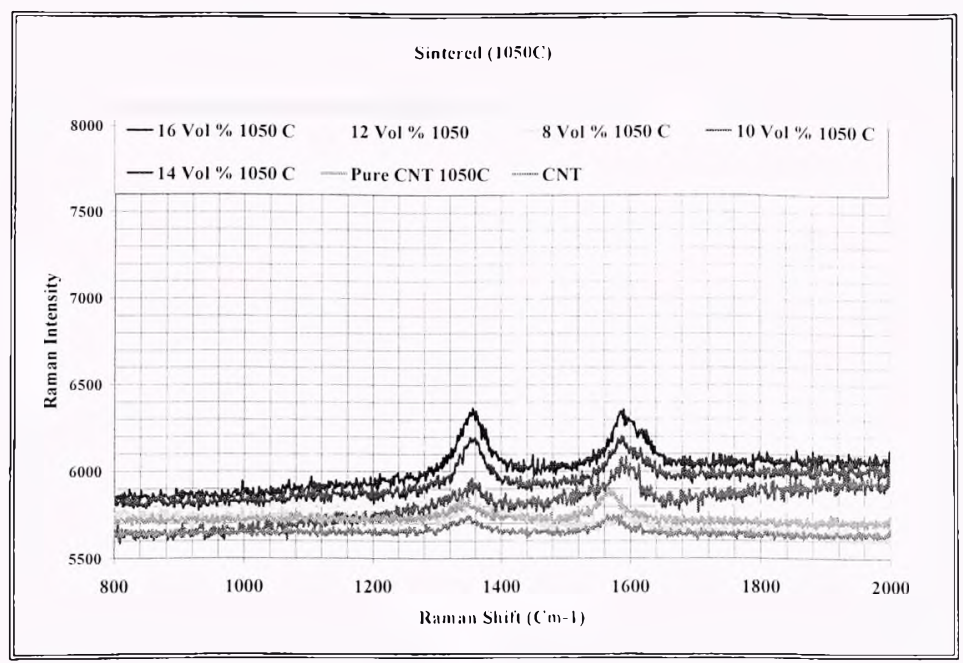

Figure 8.21: Raman spectra of composites sintered at $1050^{\circ} \mathrm{C}$.

Figure 8.22 , shows the variation of intensity ratio with an increase in volume percentage of MWCNT. The intensity ratio of $\mathrm{Cu}^{\mathrm{u}} \mathrm{Cr} 10 \mathrm{Vol} . \% \mathrm{MWCNT}$ appeared to decrease, which indicated some degree of damage to the MWCNTs.

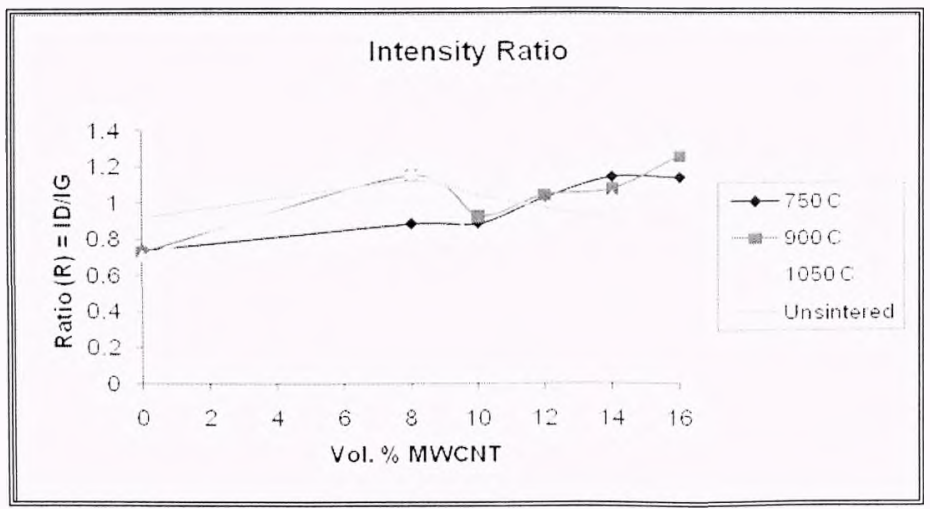

Figure 8.22: Variation of intensity ratio with MWCNT Vol, \% at three different sintering temperatures. 


\section{MORPHOLOGY}

\subsection{Scanning Electron Microscopy Analysis}

The morphology of unsintered and sintered (i.e. $750{ }^{\circ} \mathrm{C}, 900{ }^{\circ} \mathrm{C}$ and $1050{ }^{\circ} \mathrm{C}$ ) compacts was studied by SEM, and SEM/EDS and mapping analyses. The photomicrographs of these compacts are shown in Figure 9.1-9.13

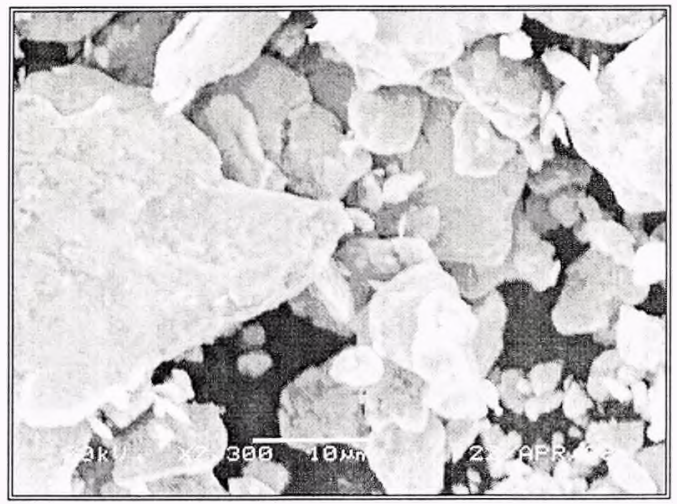

Figure 9.1: SEM image of the ball milled powder composite with Cu-Cr 8 Vol. \% MWCNT.

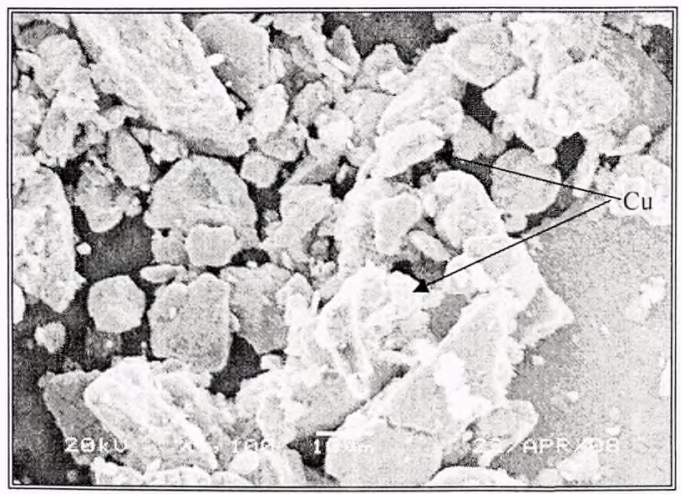

Figure 9.2: SEM image of the ball milled powder composite with Cu-Cr $12 \mathrm{Vol}$. \% MWCNT. 


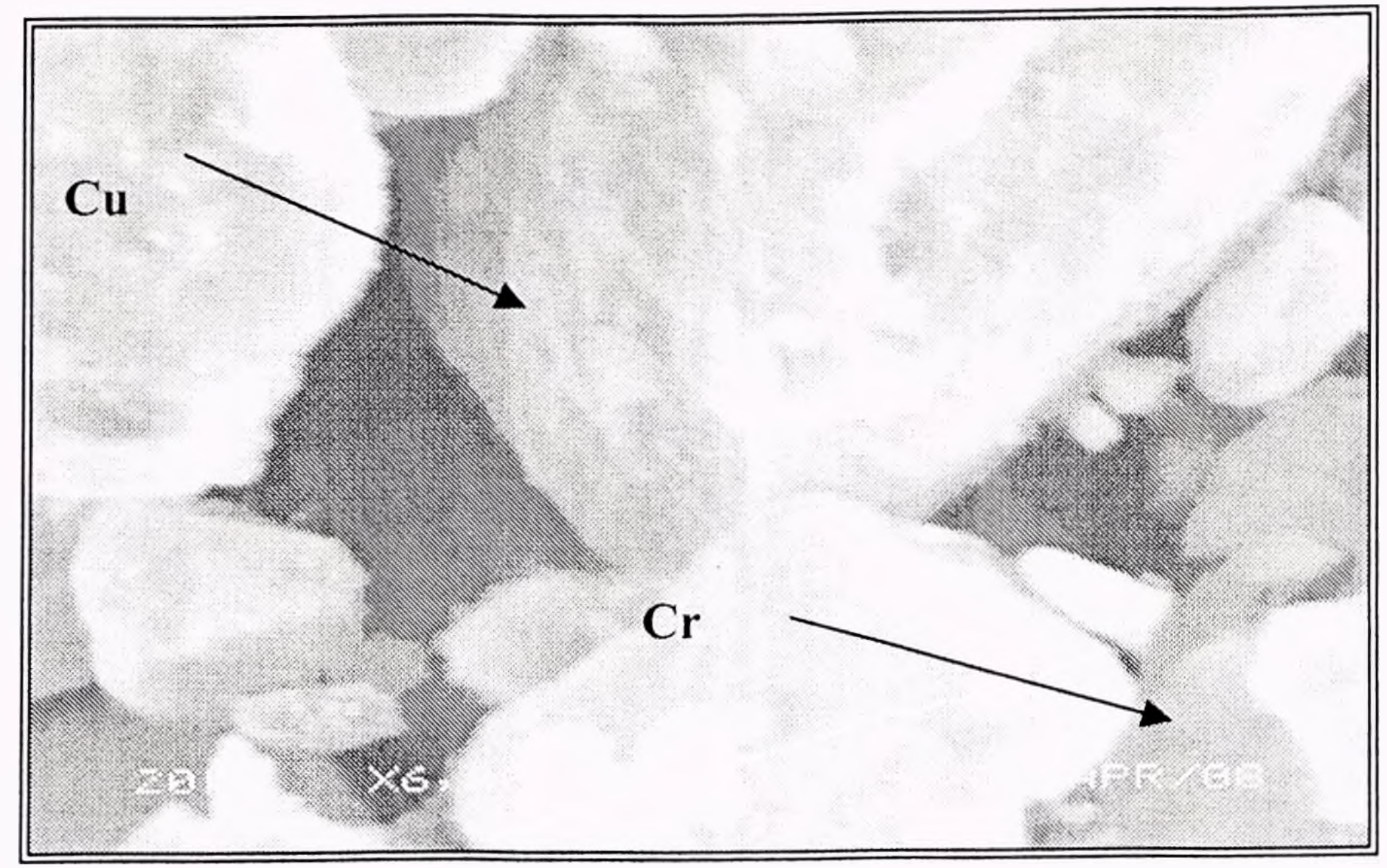

Figure 9.3: SEM image of the ball milled powder composite with Cu-Cr 14 Vol. \% MWCNT.

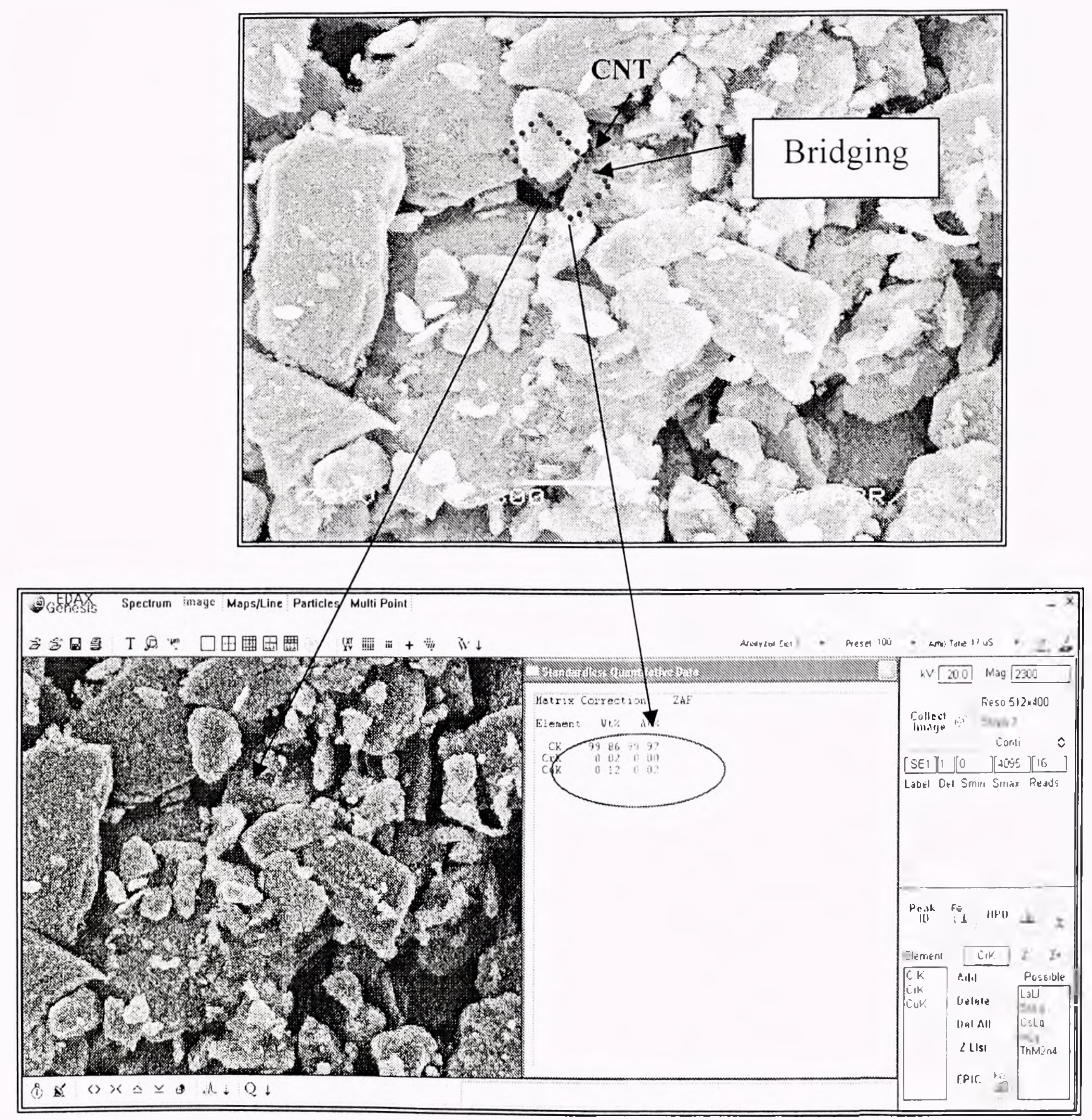

Figure 9.4: SEM and EDS analyses of the Cu-Cr 16 Vol. \% MWCNT composite. 


\subsection{SEM/EDS and Mapping Analyses of samples}

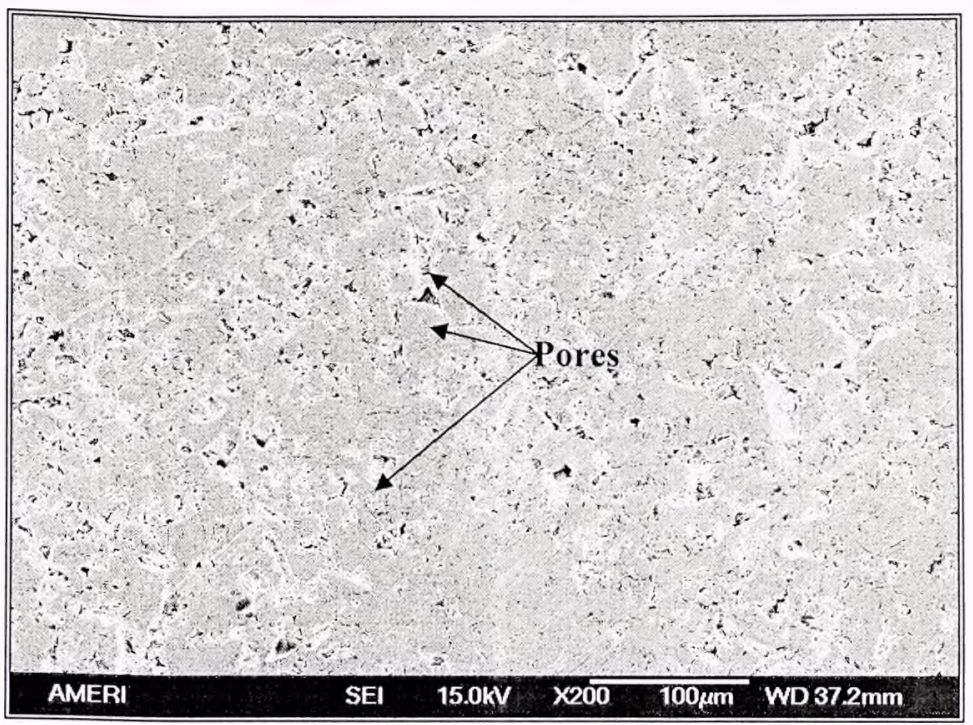

Figure 9.5: Low magnification SEM morphology images of $\mathrm{Cu}-\mathrm{Cr} 15 \mathrm{wt} \%$

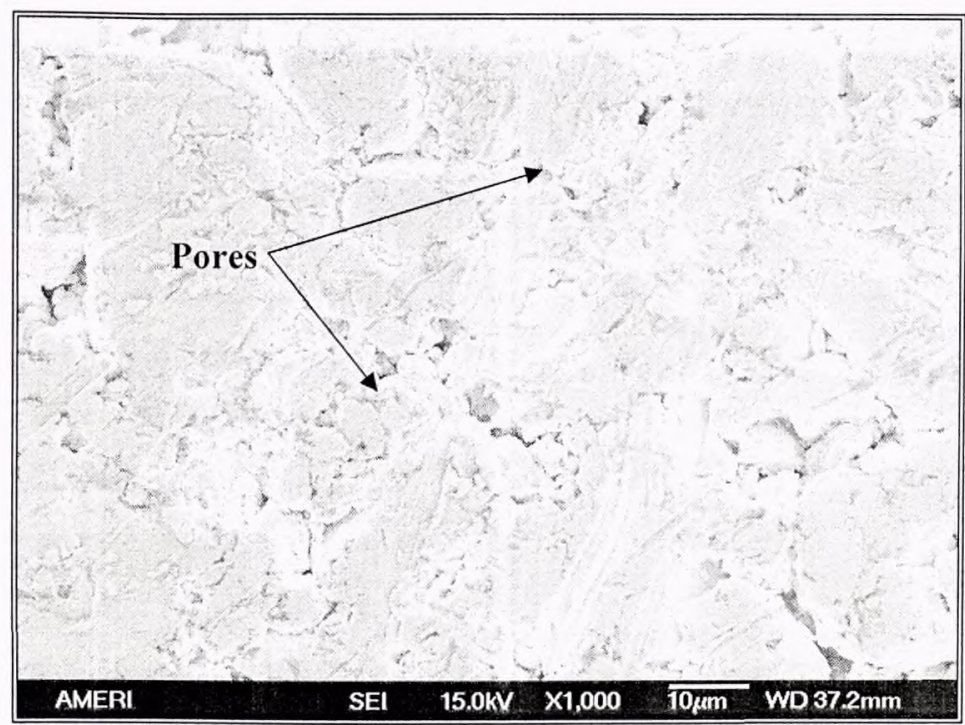

Figure 9.6: SEM image showing pores of $\mathrm{Cu}-\mathrm{Cr} 15 \mathrm{wt} . \%$ composite sintered at $750{ }^{\circ} \mathrm{C}$. 


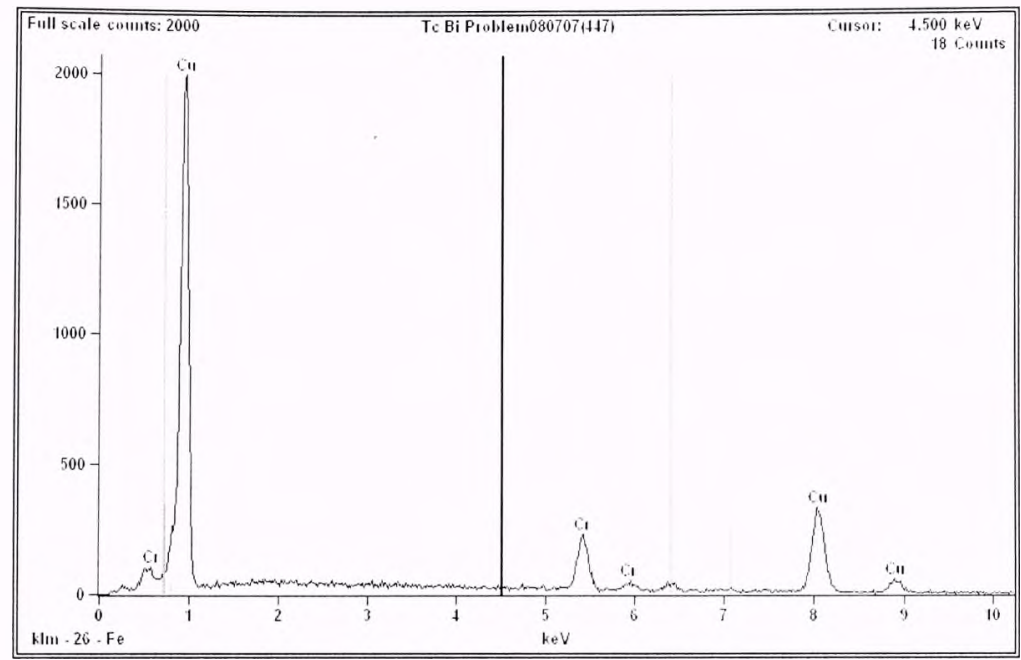

\begin{tabular}{|c|c|c|c|c|}
\hline Element Line & Weight \% & Atom \% & Formula & Compnd \% \\
\hline $\operatorname{CrK}$ & 15.15 & 17.91 & $\mathrm{Cr}$ & 15.15 \\
\hline$C r L$ & $\ldots$ & $\ldots$ & & $\ldots$ \\
\hline$C u K$ & 84.85 & 82.09 & $\mathrm{Cu}$ & 84.85 \\
\hline$C u L$ & ב-.. & $\begin{array}{c}-- \\
\end{array}$ & & $\ldots$ \\
\hline Total & 100.00 & 100.00 & & 100.00 \\
\hline
\end{tabular}

Figure 9.7: EDS analysis of $\mathrm{Cu}-\mathrm{Cr} 15 \mathrm{wt} . \%$ compact sintered at $750^{\circ} \mathrm{C}$.
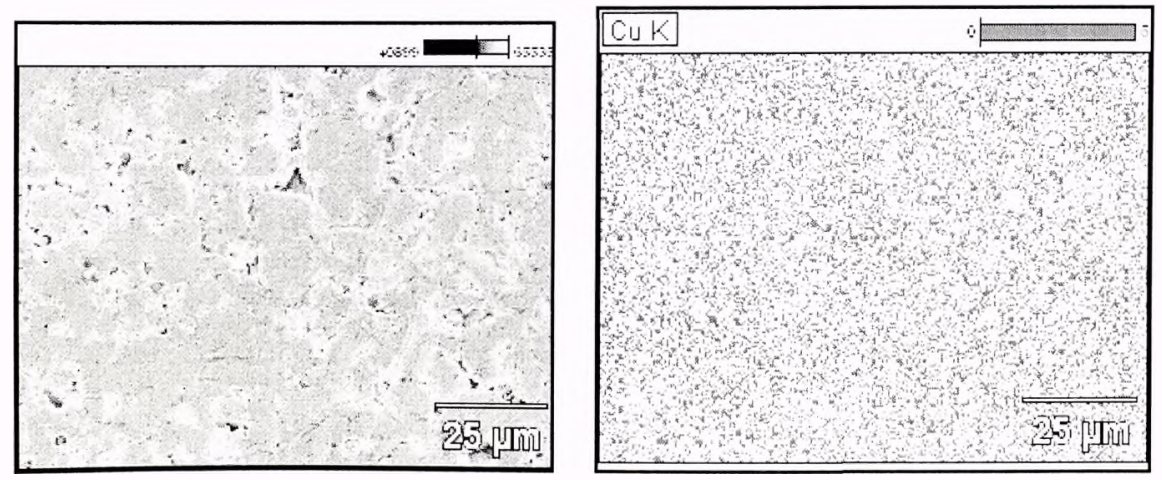


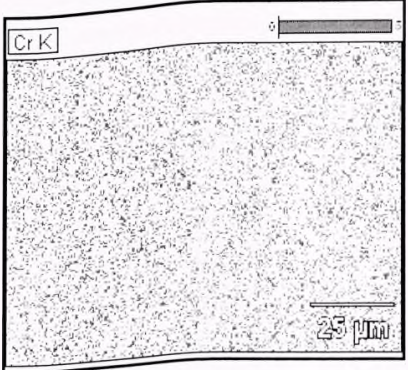

Figure 9.8: Mapping of $\mathrm{Cu}-\mathrm{Cr} 15$ wt. \% compact sintered at 750 "C illustrating homoueneity of the composite

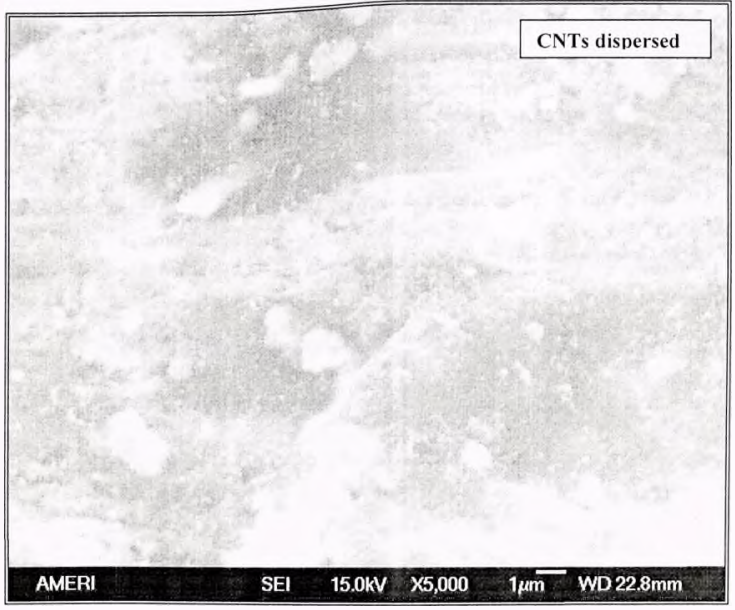

Figure 9.9: SFM image showing MWCNT dispersion in Cu-Cr 8 Vol. \% MWCNT compact sintered at $750^{\circ} \mathrm{C}$, illustrating the dispersion of MWCNT. 

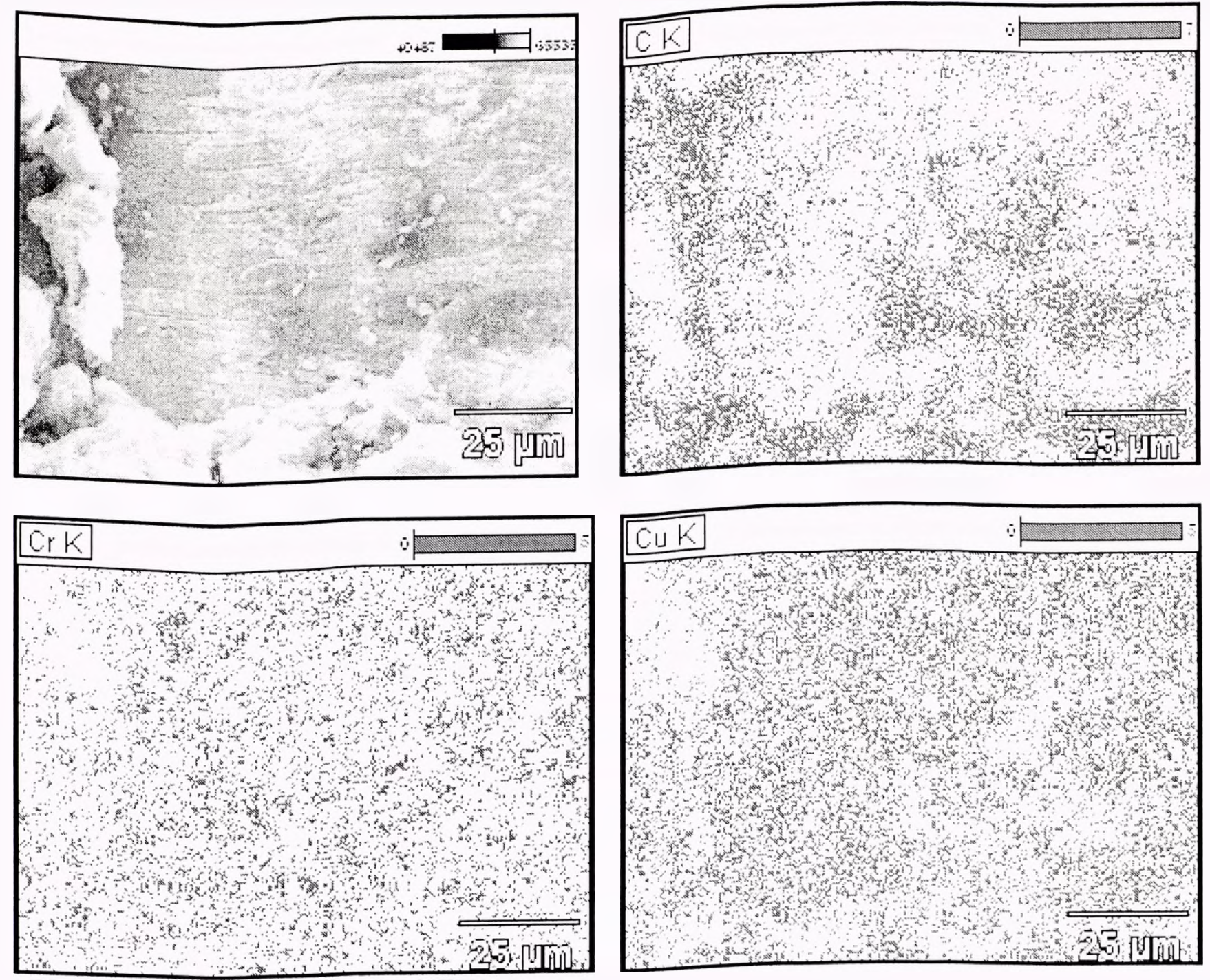

Figure 9.10: Mapping analysis for $\mathrm{Cu}-\mathrm{Cr} 8 \mathrm{Vol}$ \% MWCNT composite sintered at 750 ${ }^{\circ} \mathrm{C}$.

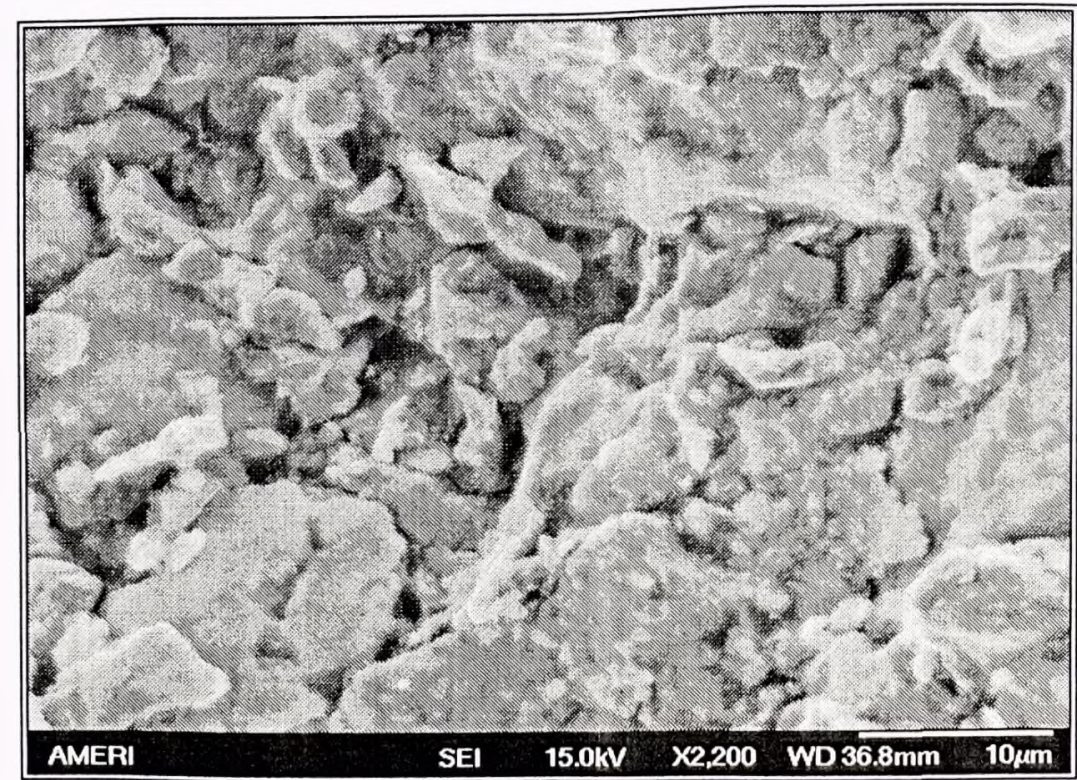

Figure 9.11: SEM photomicrograph of Cu-Cr $10 \mathrm{Vol} \%$ MWCNTs sintered at $750{ }^{\circ} \mathrm{C}$. 


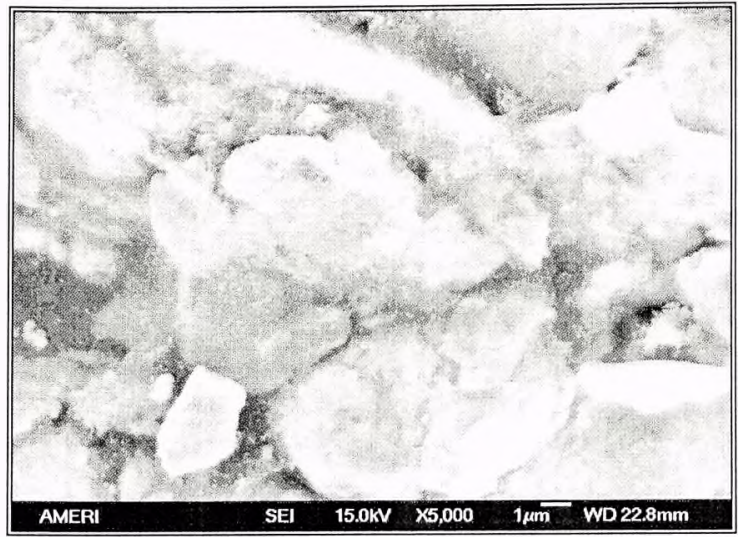

Figure 9.12: SEM image of $\mathrm{Cu}-\mathrm{Cr} 12 \mathrm{Vol} . \% \mathrm{MWCNT}$ composite sintered at $750^{\circ} \mathrm{C}$.

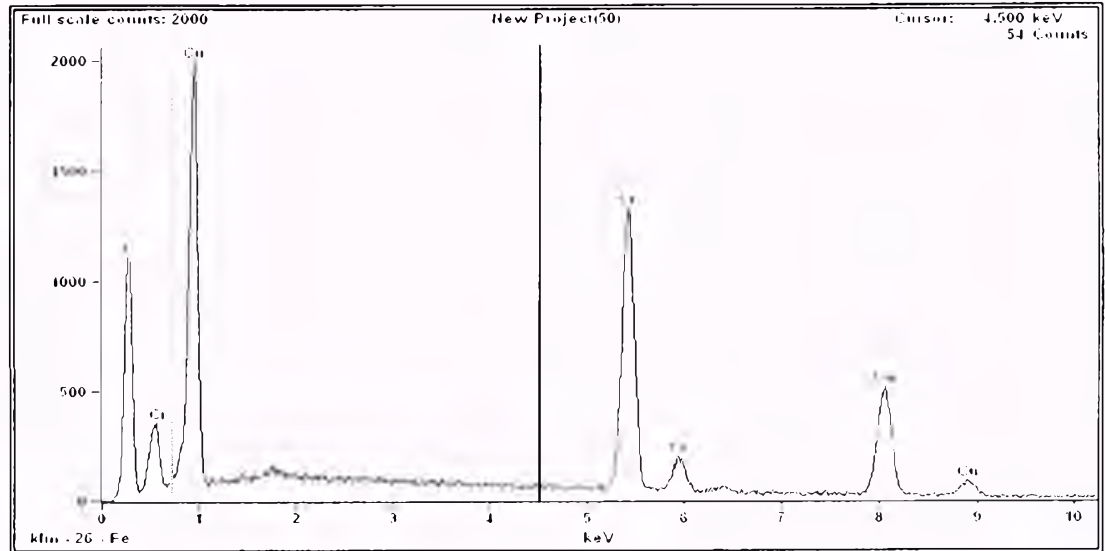

\begin{tabular}{|c|c|c|c|c|}
\hline Element Line & Weight \% & Atom \% & Formula & Compnd \% \\
\hline$\overline{C K}$ & 64.20 & 89.67 & $\mathrm{C}$ & 64.20 \\
\hline CrK & 14.99 & 4.84 & $\mathrm{Cr}$ & 14.99 \\
\hline$C r L$ & --- & $-\ldots$ & & $\ldots$ \\
\hline$C u K$ & 20.82 & 5.50 & $\mathrm{Cu}$ & 20.82 \\
\hline$C u L$ & $\cdots$ & $-\cdots$ & & -.- \\
\hline Total & 100.00 & 100.00 & & 100.00 \\
\hline
\end{tabular}

Figure 9.13: EDS data of $\mathrm{Cu}-\mathrm{Cr} 12 \mathrm{Vol} . \% \mathrm{MWCNT}$ composite sintered at $750^{\circ} \mathrm{C}$. 
It can be deduced from the elemental mapping and the phase diagrams that $\mathrm{Cu}, \mathrm{Cr}$ and $\mathrm{CNT}$ do not form a solid solution. From a thermodynamic perspective some chromium carbide can be expected to be formed ( $\Delta \mathrm{G}=-15000 \mathrm{cal} / \mathrm{mol}$ ). However, $\mathrm{XRT}$ ) analysis did not reveal its presence. Copper is incorporated as solute in the chromium matrix as does chromium in the copper matrix. It is postulated that the highly reactive edges and broken surfaces of the MWCNT serve as reactive sites for the formation of carbides, resulting in various bonding mechanism like MWCNT chain-like structures, MWCNT Y-junctions, MWCNT rope anchors and MWCNT bridges. The MWCNT bonds with chromium as a carbide at the interface, whereas some chromium at the interface become engulfed as solute in the copper matrix. These interfacial mechanisms are believed to be mechanism are believed to be responsible for the mechanical properties of the composite.

Figure 9.14, shows the elemental mapping analysis of $\mathrm{Cu}-\mathrm{Cr} 12$ Vol. \% MWCNT composite sintered at $750^{\circ} \mathrm{C}$. Superimposed are $\mathrm{C}-\mathrm{Cu}, \mathrm{Cu}-\mathrm{Cr}$ and $\mathrm{C}-\mathrm{Cr}$ phase diagrams, which illustrate that $\mathrm{C}$ is insoluble in $\mathrm{Cu}$. and that $\mathrm{C}$. $\mathrm{Cu}$ and $\mathrm{Cr}$ do not form solid solutions with each other. This feature is illustrated in the encircled areas on the clemental mapping where elements do not overlap. 


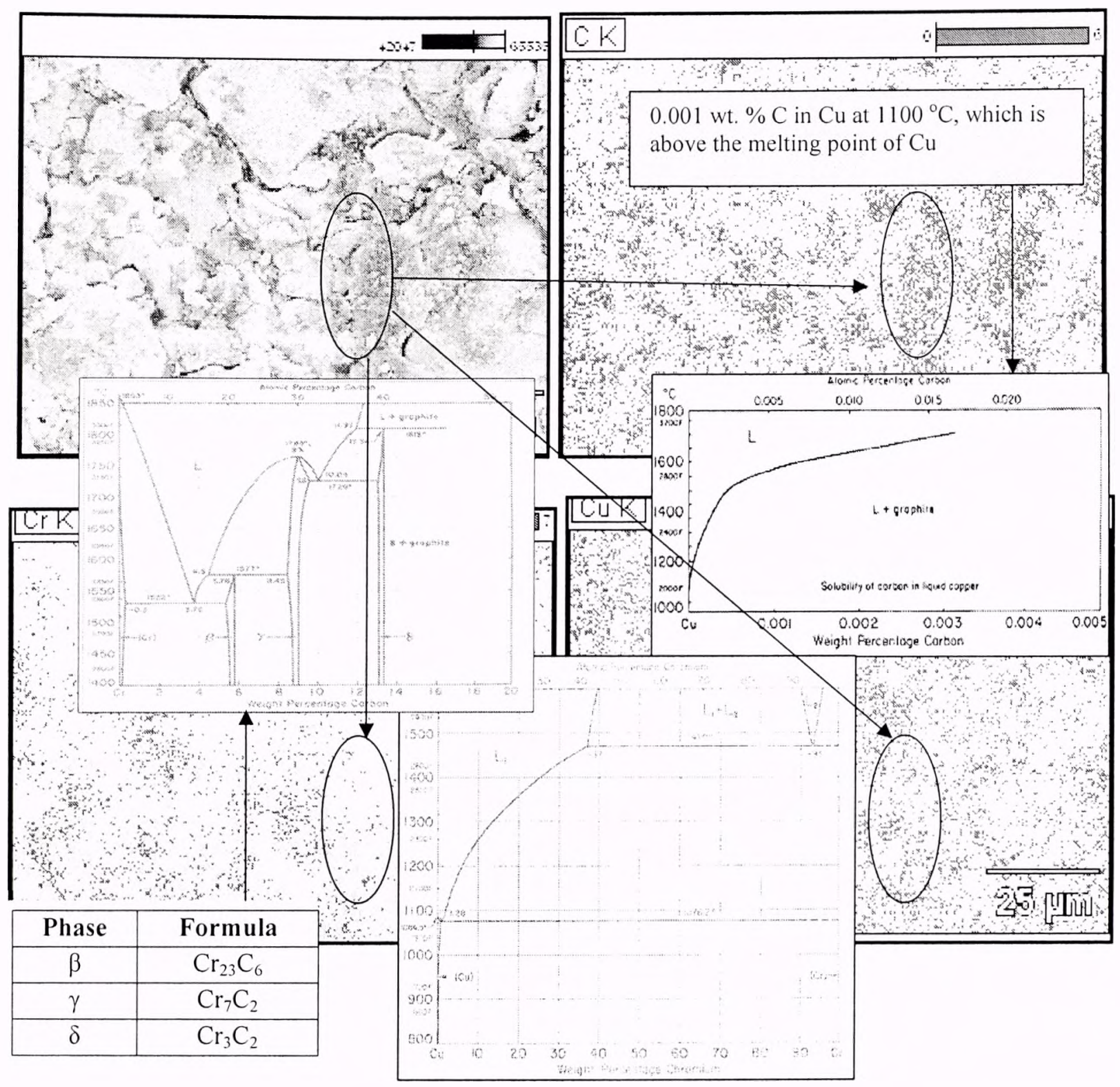

Figure 9.14: Mapping of Cu-Cr $12 \mathrm{Vol} . \% \mathrm{MWCNT}$ composite sintered at $750{ }^{\circ} \mathrm{C}$. 


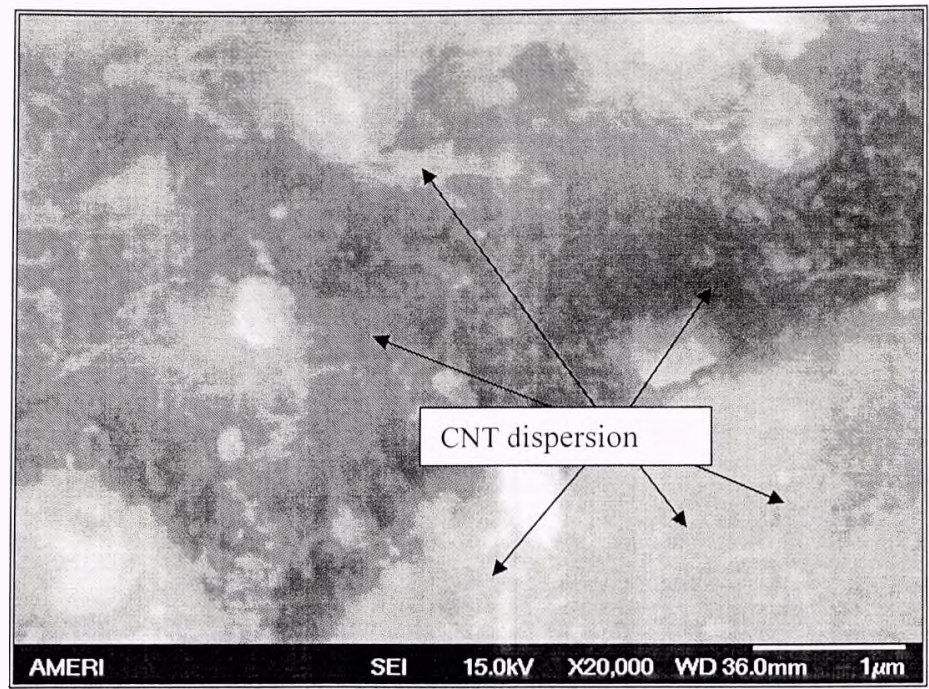

Figure 9.15: SEM analyses of $\mathrm{Cu}-\mathrm{Cr} 14 \mathrm{Vol} . \% \mathrm{MWCNT}$ composite sintered at $750{ }^{\circ} \mathrm{C}$.

Figure 9.16 shows the elemental mapping analysis of Cu-Cr 14 Vol. \% MWCNT composite. It can be seen that the $\mathrm{Cu}$ and $\mathrm{Cr}$ are homogeneously dispersed, but MWCNTs were not. In fact, some agglomeration of MWCNTs was observed within the composite. Feng et. al. (2005), discussed the agglomeration of CNTs in Ag matrix composites reinforced with high concentrations of CNTs. The high surface energy of interfaces causes agglomeration of the CNTs resulting in very poor interfacial bonding and lower hardness [76]. 

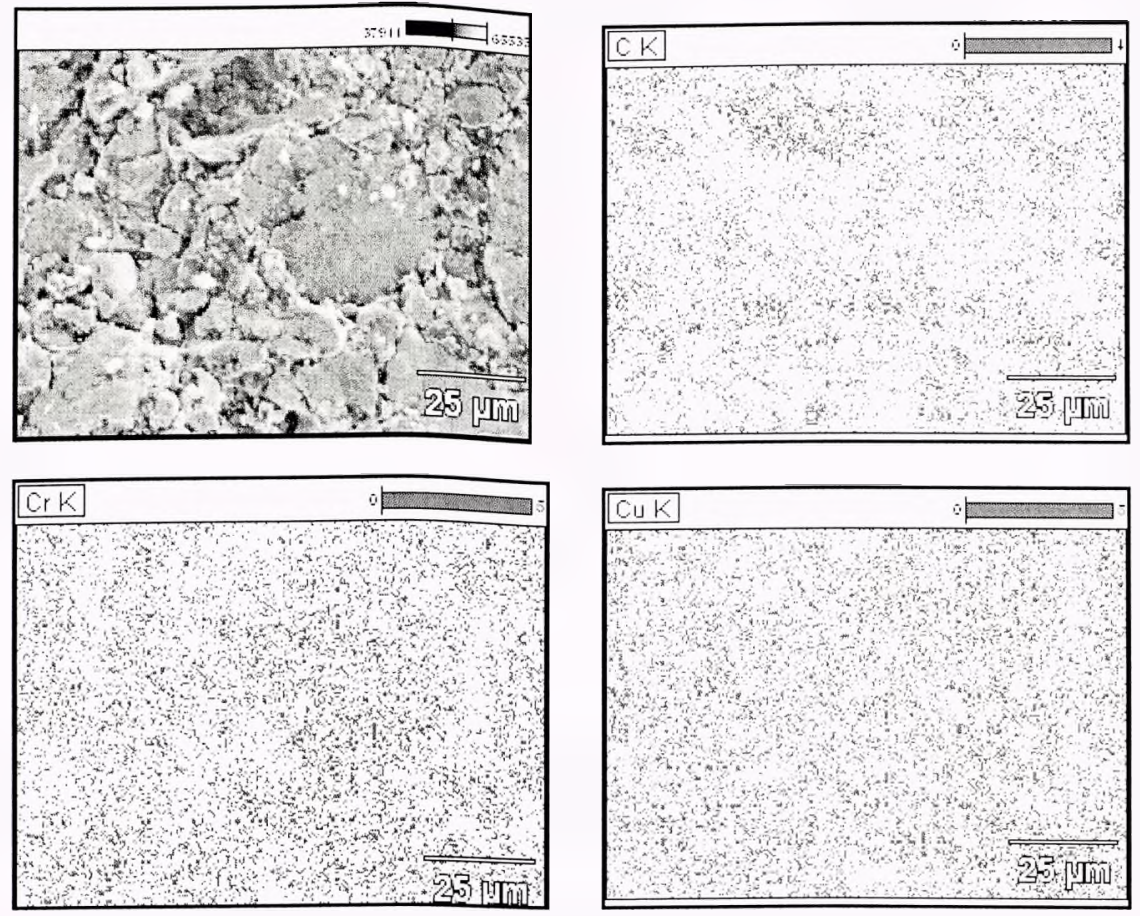

Figure 9.16: Elemental Mapping analysis of Cu-Cr 14 Vol. \% MWCNT composite sintered at $750{ }^{\circ} \mathrm{C}$.

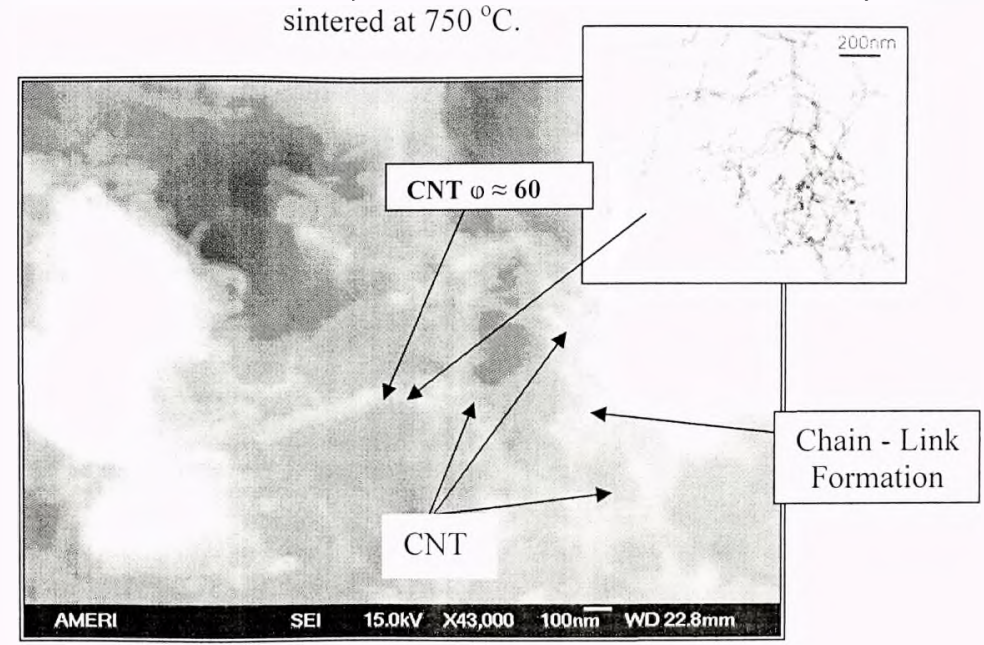

Figure 9.17: SEM image for Cu-Cr 16 Vol. \% MWCNTs, 
Comparing the SEM photomicrograph of pure MWCNT (see inset) with the photomicrograph of a composite containing 16 Vol.\% MWCNTs Figure 9.17, it can be concluded that the CNTs were not significantly damaged after 5 minutes of ball milling (1/4" balls) followed by sintering, as the dimensions of the MWCNTs remained relatively constant (i.e. $\varphi \sim 60$ ).
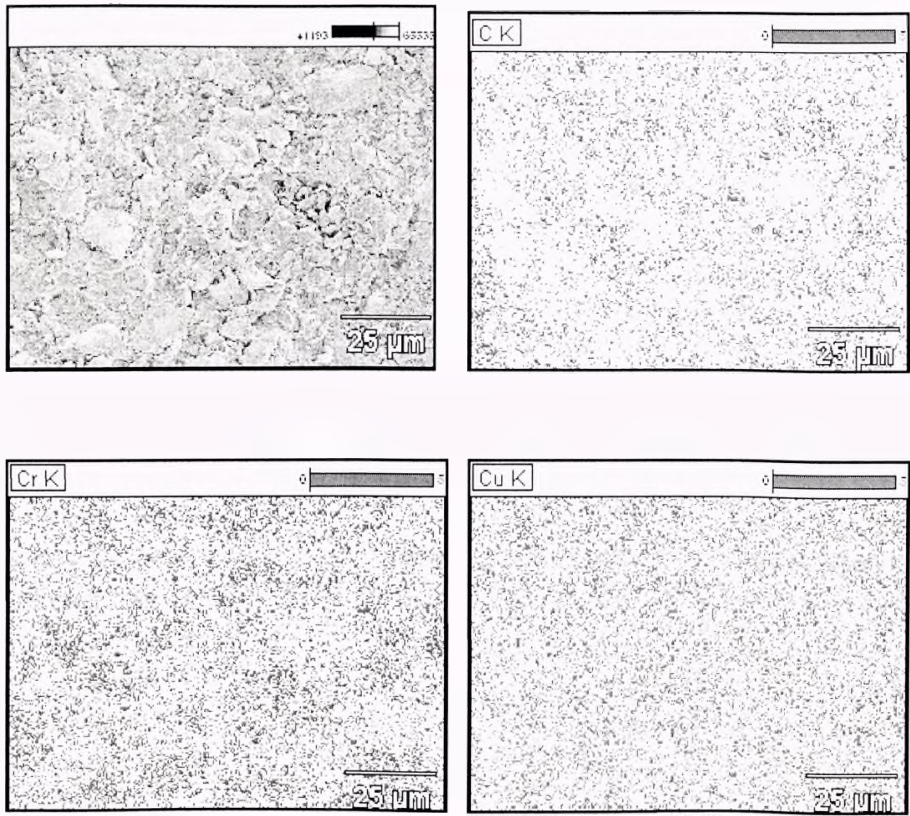

Figure 9.18: Mapping analysis of $\mathrm{Cu}-\mathrm{Cr} 16 \mathrm{Vol} . \% \mathrm{MWCNT}$ sintered at $750^{\circ} \mathrm{C}$. that Cu$\mathrm{Cr}$ are homogeneously distributed, MWCNTs were not evenly dispersed. 

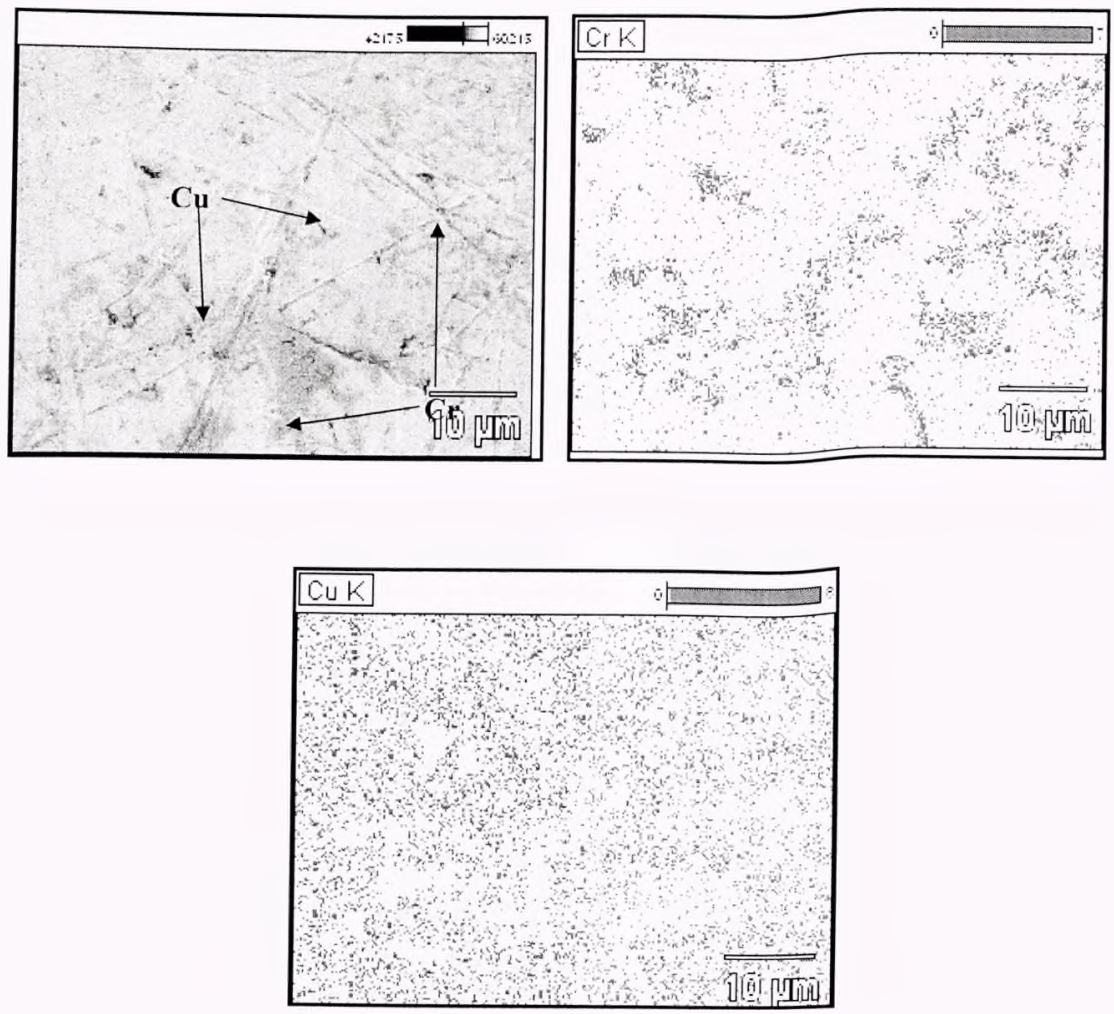

Figure 9.19: Mapping analysis of $\mathrm{Cu}-\mathrm{Cr} 15 \mathrm{wt} . \%$ sintered at $900{ }^{\circ} \mathrm{C}$.

The darker region in Figure 9.19, shows $\mathrm{Cr}$ and lighter region shows $\mathrm{Cu}$ because $\mathrm{Cr}$ has a smaller atomic weight as compared to $\mathrm{Cu}$ (i.e. $\mathrm{Cr}$ amu $=52$ and $\mathrm{Cu}$ amu $=$ 63.55), the smaller amu means more absorption resulting in darker region. 

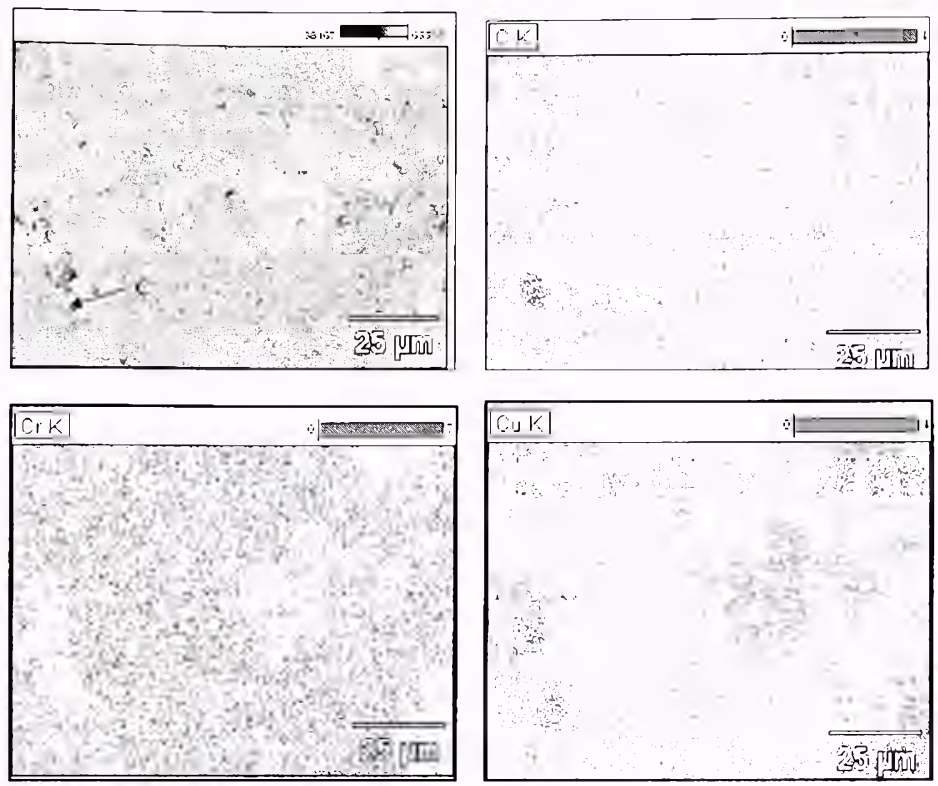

Figure 9.20: Mapping analysis of $\mathrm{Cu}-\mathrm{Cr} 8$ Vol. \% MWCNT sample.

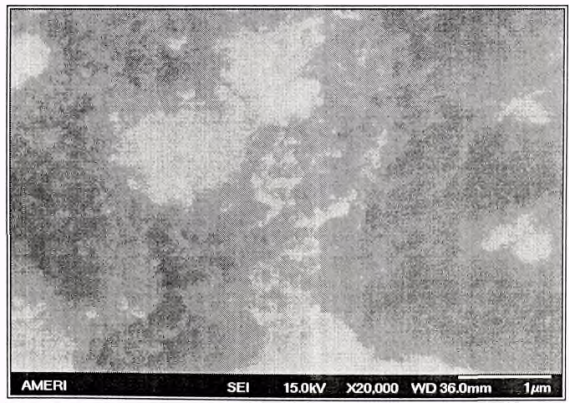

Figure9.21: SEM image showing dispersion of MWCNTs in Cu-Cr 10 Vol. \% MWCNT pellet sintered at $900^{\circ} \mathrm{C}$. 

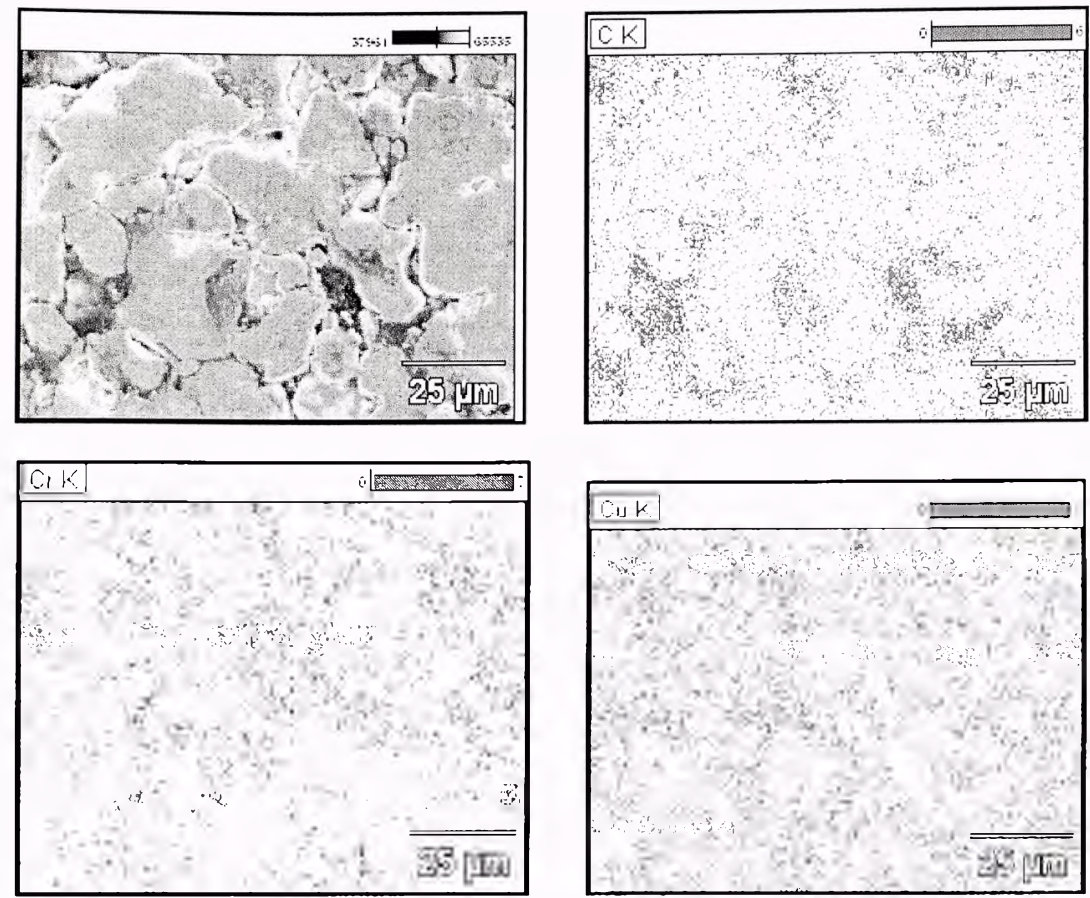

Figure 9.22: Mapping analyses of $\mathrm{Cu}-\mathrm{Cr} 10 \mathrm{Vol} . \% \mathrm{MWCNT}$ composite sintered at 900 ${ }^{\circ} \mathrm{C}$.

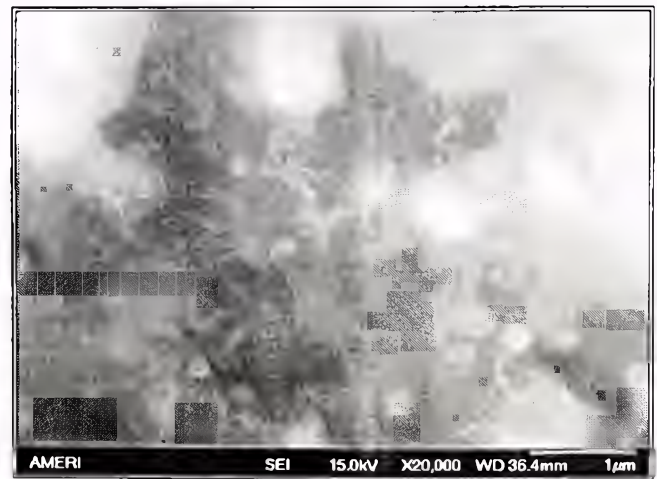

Figure 9.23: SEM image showing CNT dispersion in $\mathrm{Cu}-\mathrm{Cr} 12$ Vol. \% MWCNT composite sintered at $900{ }^{\circ} \mathrm{C}$. 


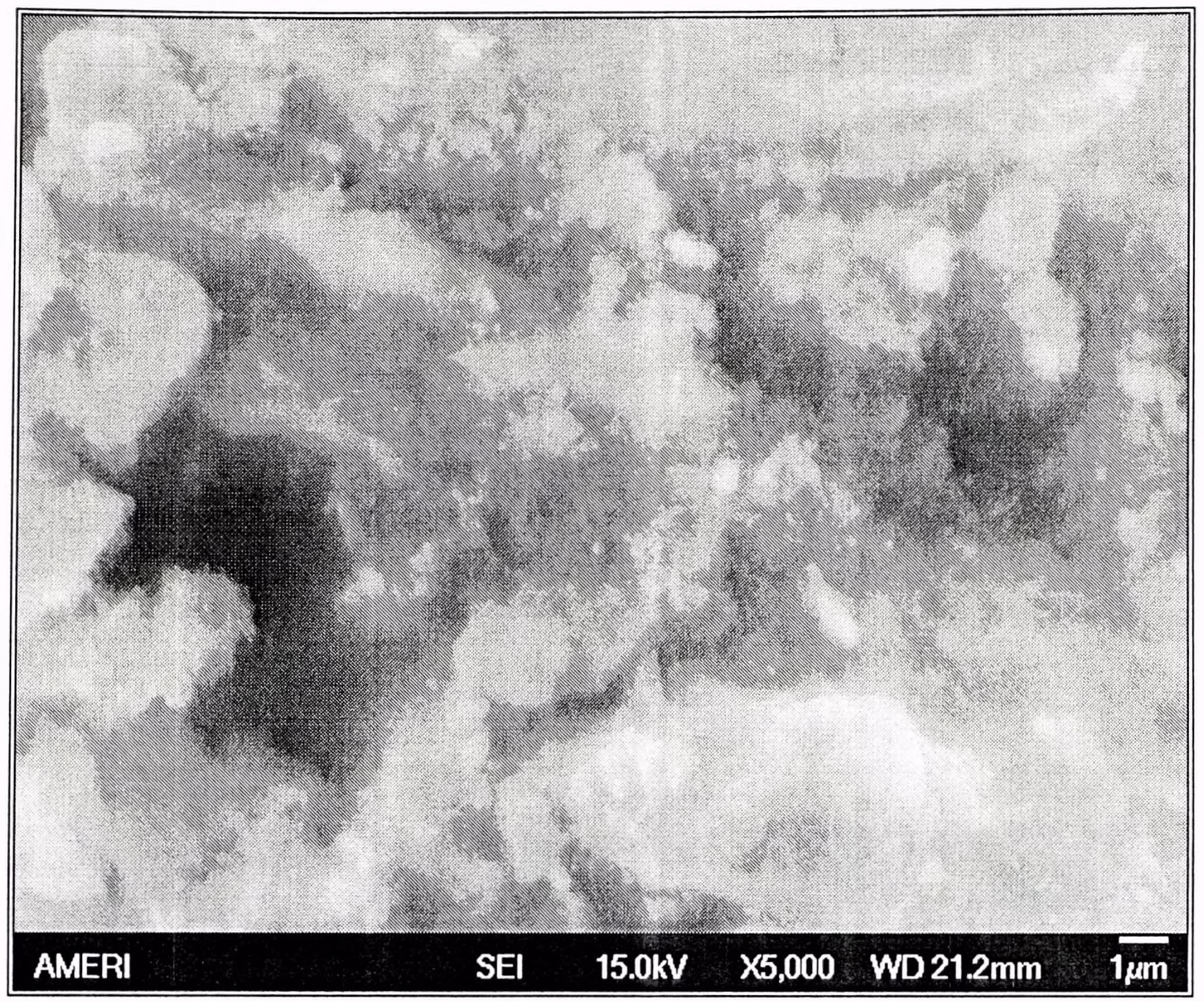

Figure 9.24: SEM image of $\mathrm{Cu}-\mathrm{Cr} 14 \mathrm{Vol}$. \% MWCNT composite sintered at $900{ }^{\circ} \mathrm{C}$.
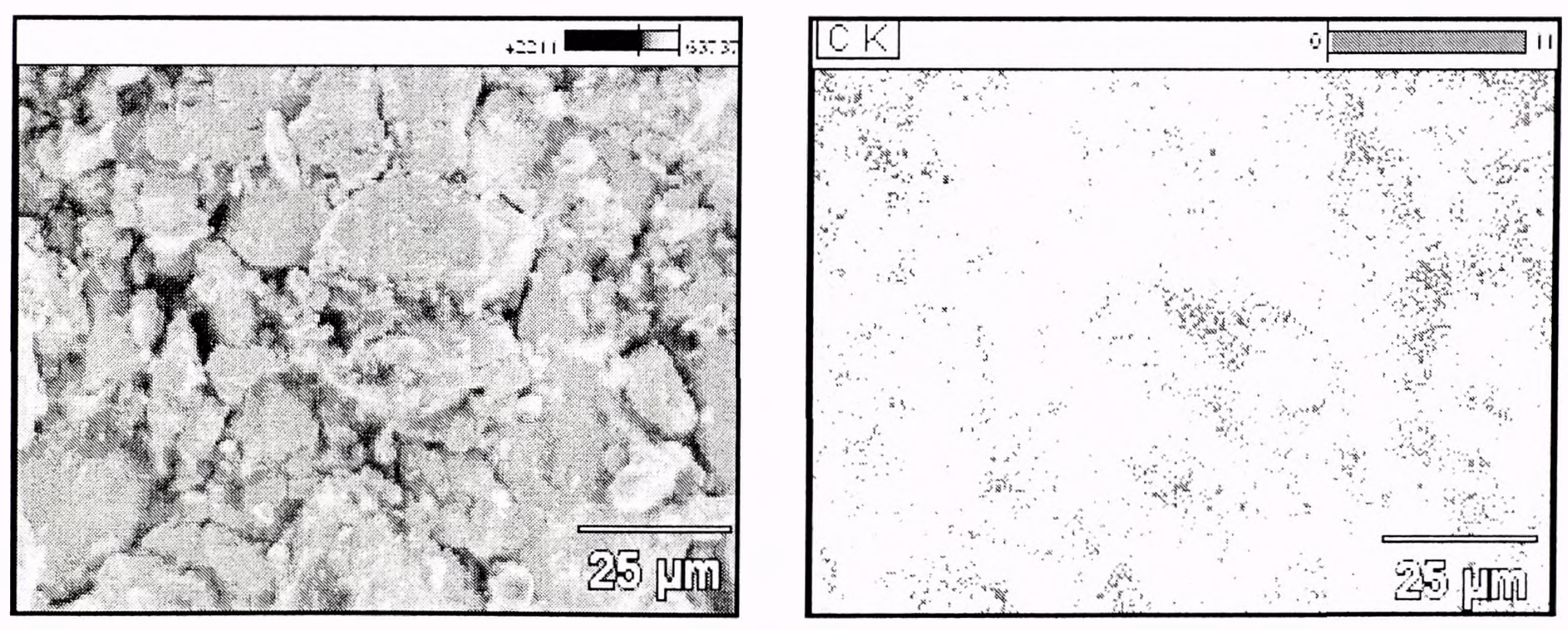

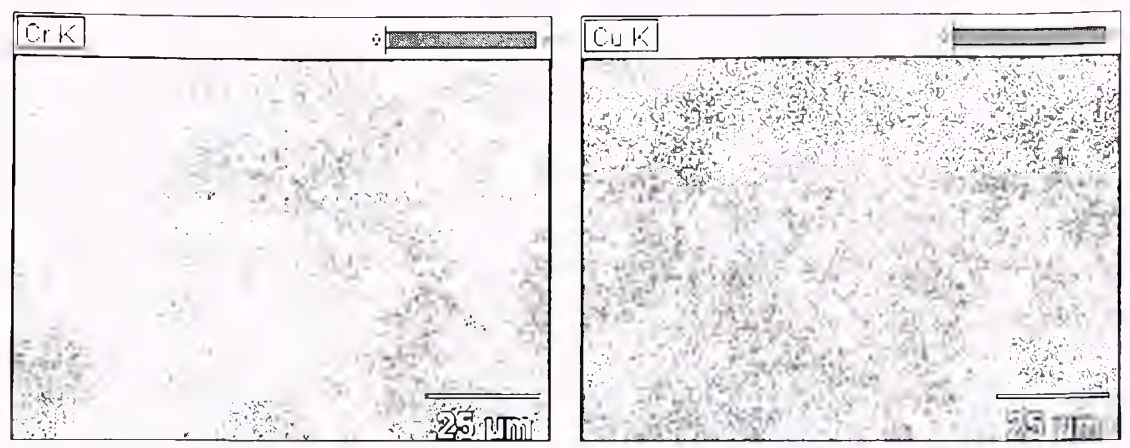

Figure 9.25: Mapping analysis showing the $\mathrm{Cu}$. $\mathrm{Cr}$ and MWCNT distribution in a $\mathrm{Cu}-\mathrm{Cr}$ 14 Vol. \% MWCNT composite sintered at $900^{\circ} \mathrm{C}$.

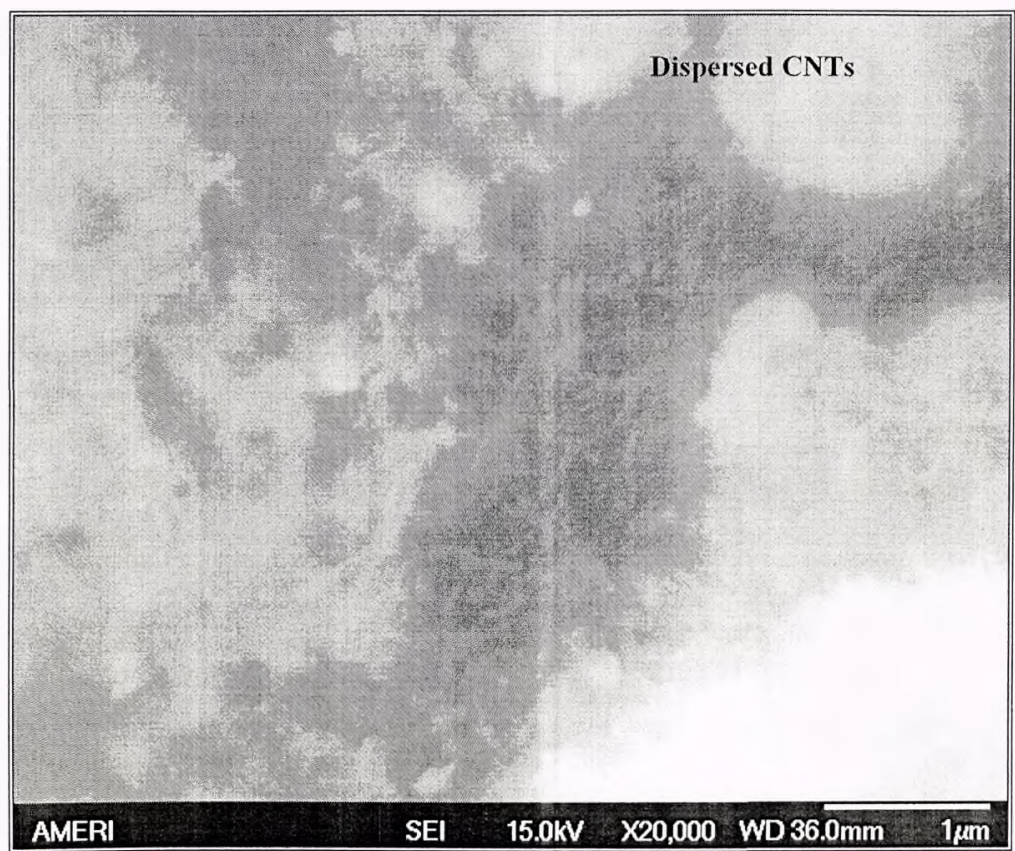

Figure 9.26: SEM analysis of Cu-Cr 16 Vol.\% MWCNT composite sintered at $900{ }^{\circ} \mathrm{C}$. 

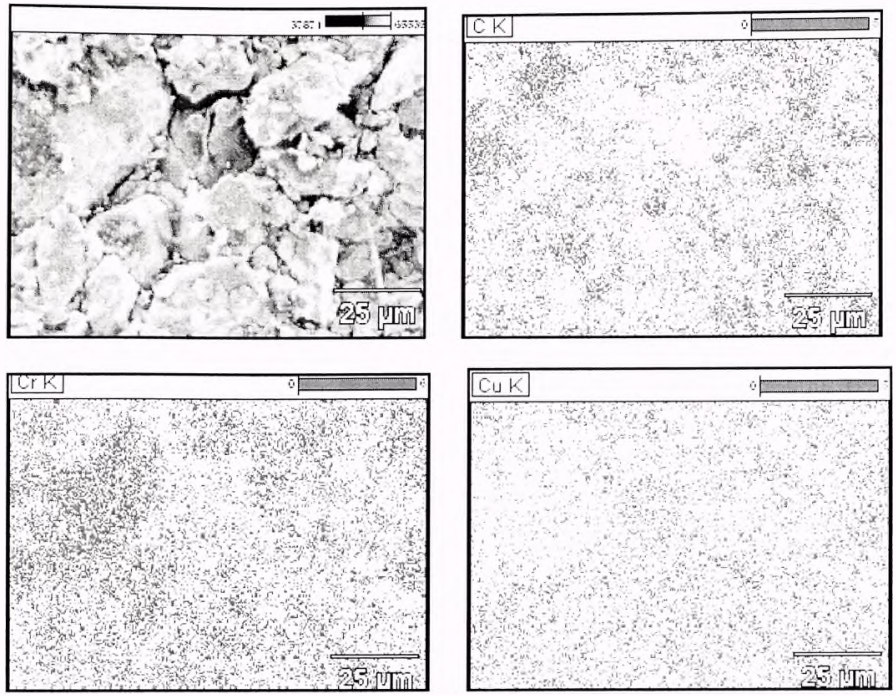

Figure 9.27: SEM and Mapping analysis of $\mathrm{Cu}-\mathrm{Cr} 16 \mathrm{Vol} \% \mathrm{MWCNT}$ 's composite sintered at $900{ }^{\circ} \mathrm{C}$

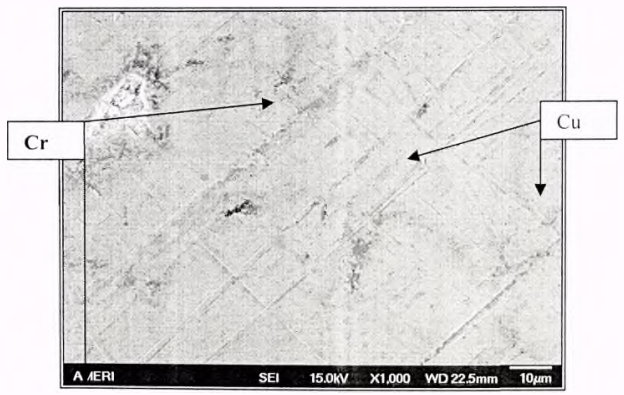

Figure 9.28: SEM analysis of $\mathrm{Cu}-\mathrm{Cr} 15 \mathrm{wt} . \%$ composite sintered at $1050^{\circ} \mathrm{C}$. 


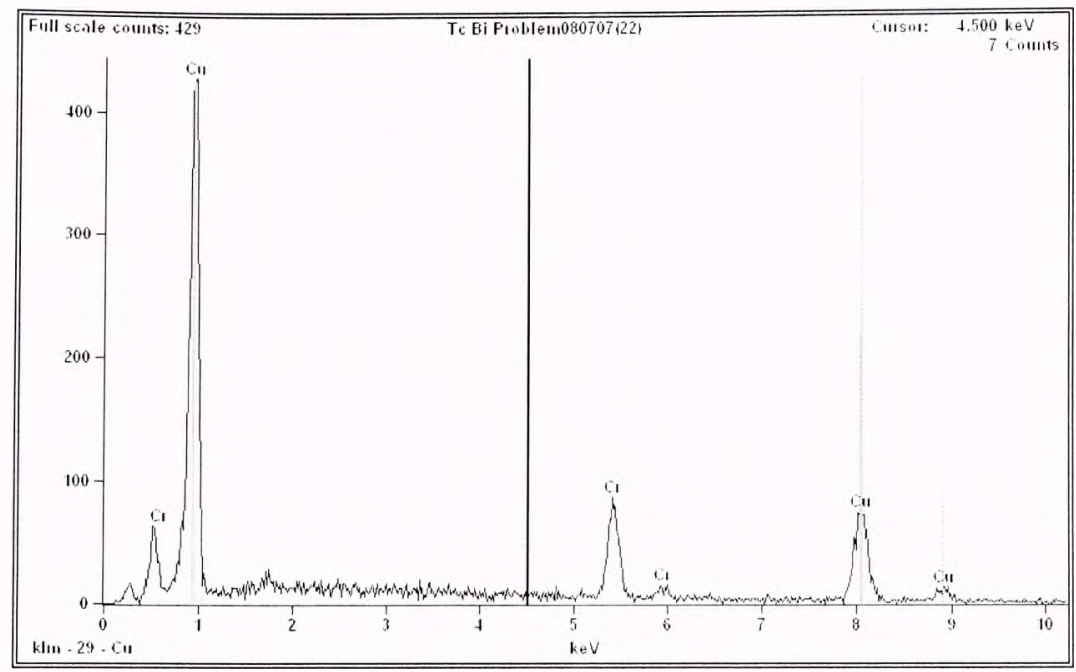

\begin{tabular}{|c|c|c|c|c|}
\hline Element Line & Weight \% & Atom \% & Formula & Compnd \% \\
\hline $\operatorname{CrK}$ & 21.29 & 24.85 & $\mathrm{Cr}$ & 21.29 \\
\hline$C r L$ & $\cdots$ & -- & & $\cdots$ \\
\hline$C u K$ & 78.71 & 75.15 & $\mathrm{Cu}$ & 78.71 \\
\hline$C u L$ & $\cdots$ & $\cdots$ & & $\cdots$ \\
\hline Total & 100.00 & 100.00 & & 100.00 \\
\hline
\end{tabular}
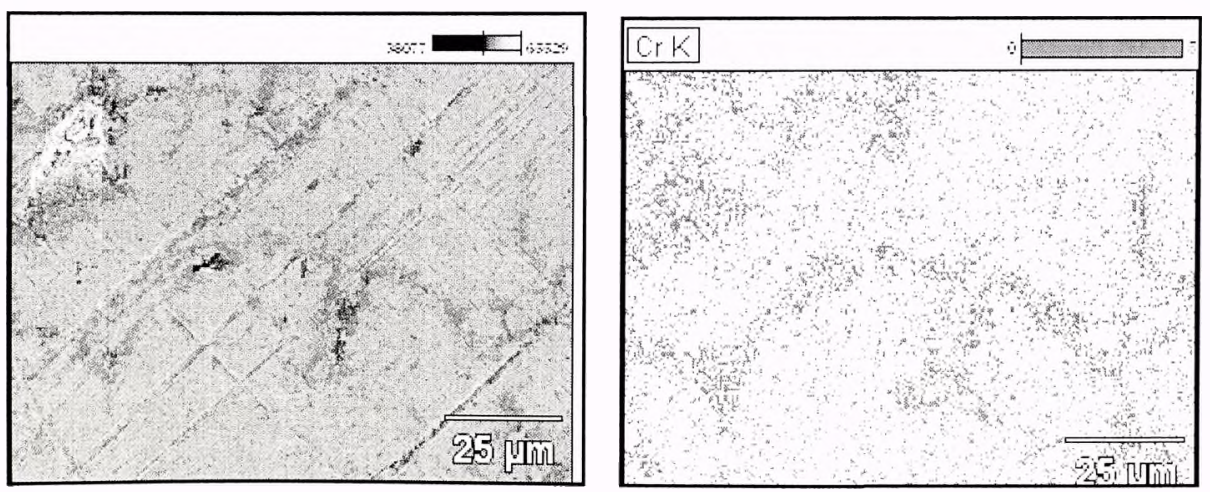


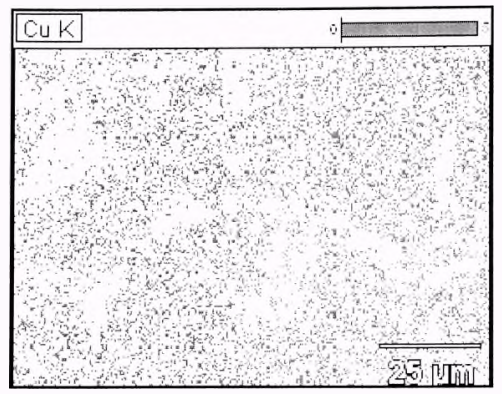

Figure 9.29: Mapping analysis of $\mathrm{Cu}-\mathrm{Cr} 15 \mathrm{wt} . \%$ composite sintered at $1050^{\circ} \mathrm{C}$

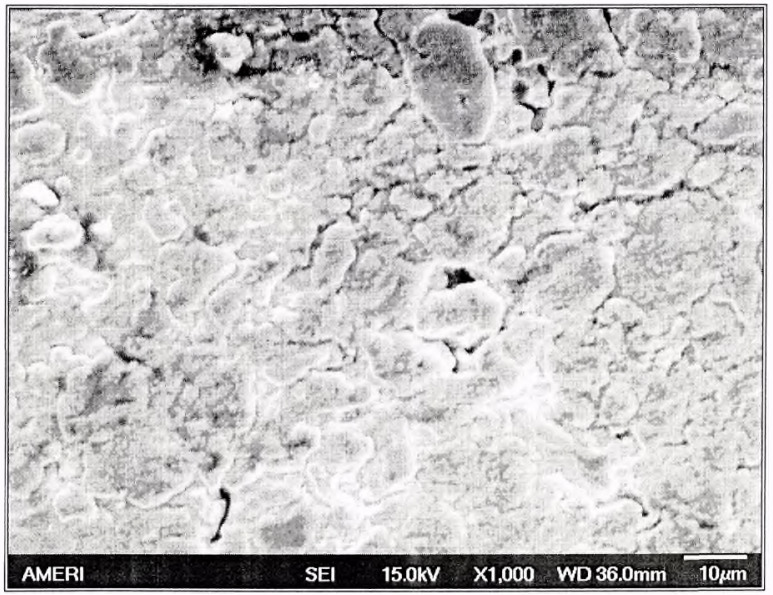

Figure 9.30: SEM image of $\mathrm{Cu}-\mathrm{Cr} 8 \mathrm{Vol} . \% \mathrm{MWCNT}$ composite sintered at $1050^{\circ} \mathrm{C}$. 


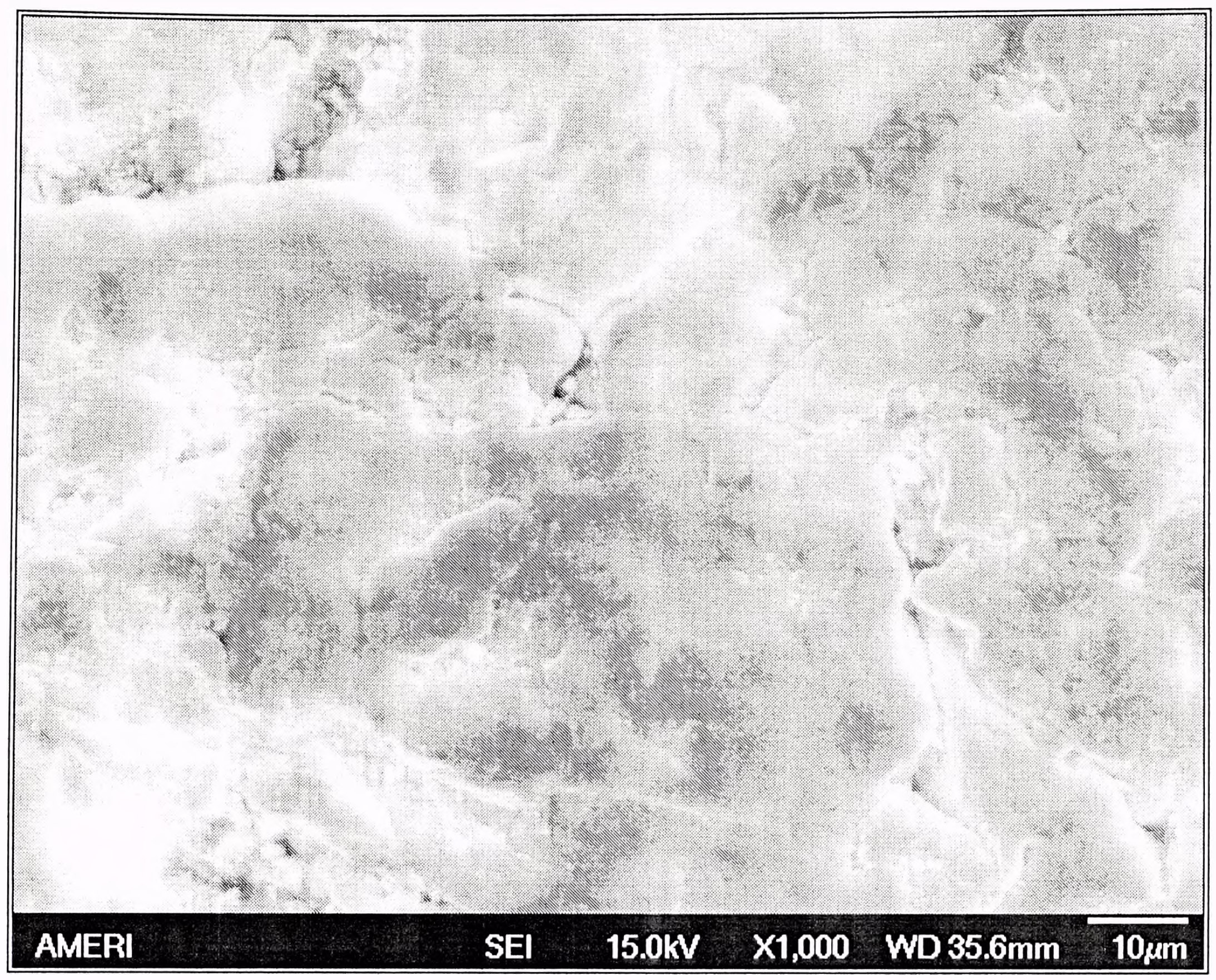

Figure 9.31: SEM images of Cu-Cr $10 \mathrm{Vol} . \%$ MWCNT composite sintered at $1050^{\circ} \mathrm{C}$.
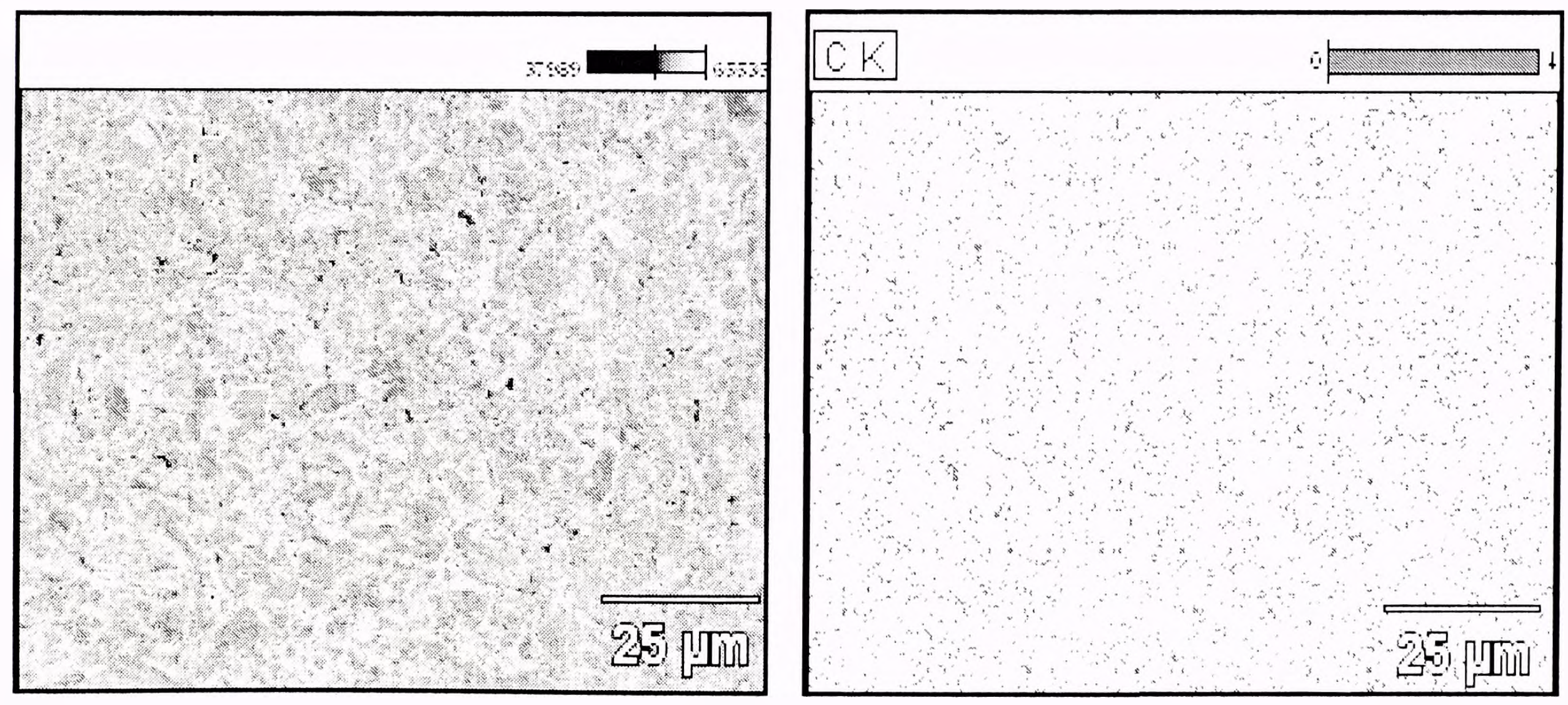

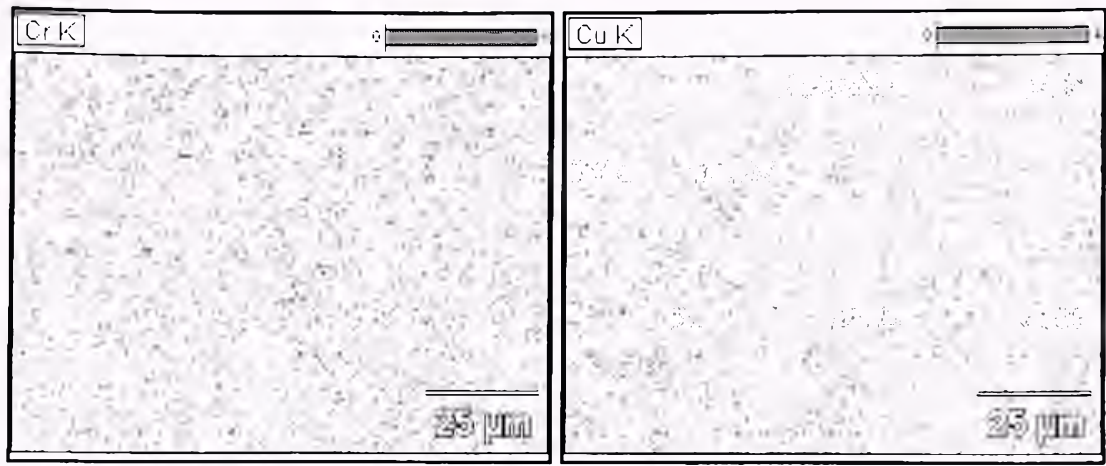

Figure 9.32: Mapping results of $\mathrm{Cu}-\mathrm{Cr} 10 \mathrm{Vol} . \% \mathrm{MWCNT}$ composite sintered at $1050^{\circ} \mathrm{C}$ shows that the three phases are homogeneous.

Elemental mapping analysis for composites sintered at $1050{ }^{\circ} \mathrm{C}$ revealed more $\mathrm{Cr}$ which lead to a possibility that there was $\mathrm{Cr}$ precipitation ( $\mathrm{Cu}$ atomic radius $\mathrm{R}_{\mathrm{Cu}}$ $=1.2782 \mathrm{~A}^{0}, R_{C r}=1.2490 \mathrm{~A}^{\circ}$ ). The $\mathrm{Cr}$ atom diffuse as solute atoms into the $\mathrm{Cu}$ matrix and the Cu matrix lattice parameter (crystal structure) is reduced. Thus the solidification of any molten $\mathrm{Cu}$ matrix containing $\mathrm{Cr}$ solute, results in the precipitation of $\mathrm{Cr}$ causes the Cu lattice parameter to become closer to that of pure Cu and the electrical conductivity is enhanced. This is due to Cu having an FCC crystal structure, whereas Cr has a BCC lattice structure. 

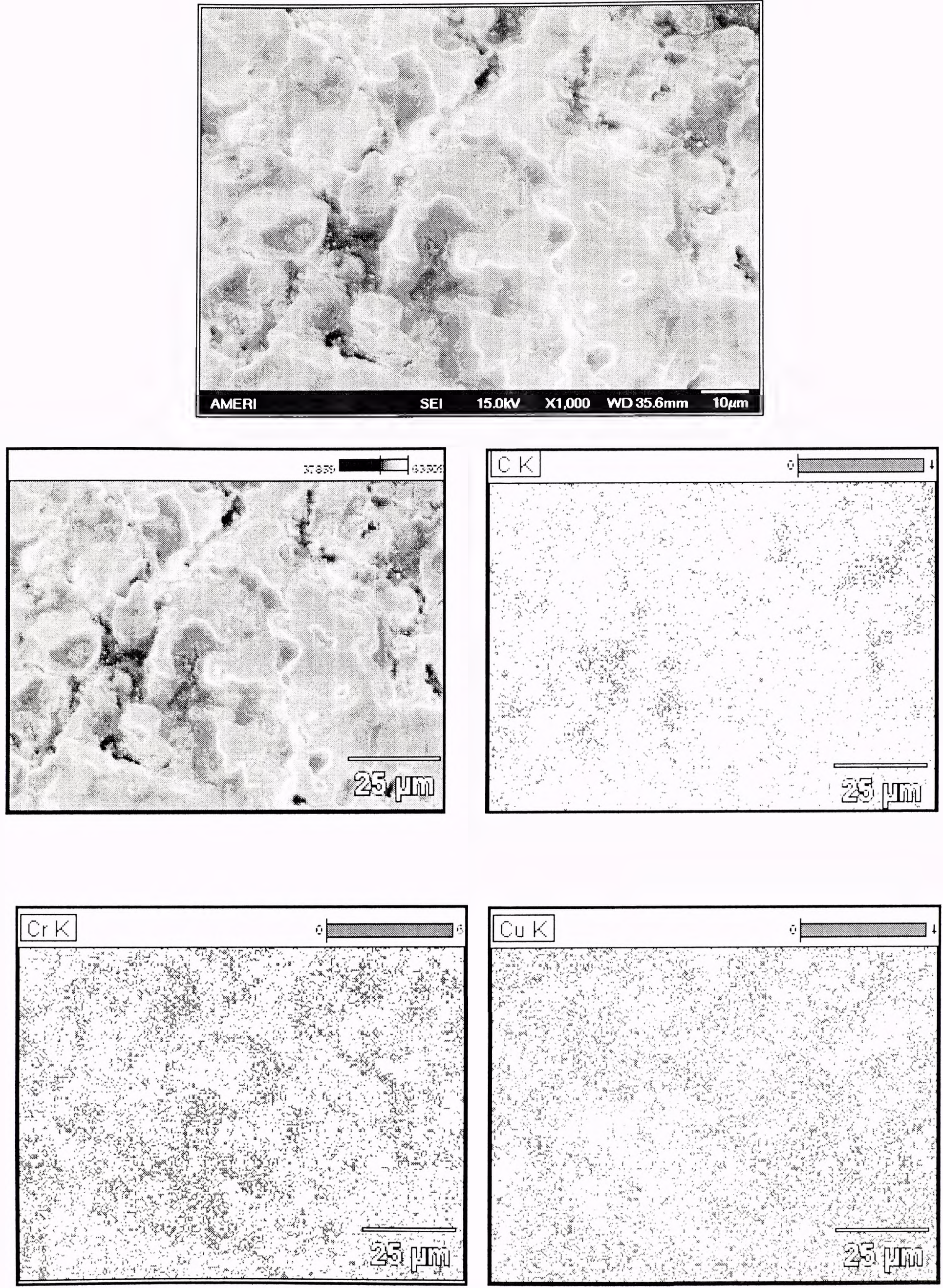

Figure 9.34: SEM and Mapping analysis of $\mathrm{Cu}-\mathrm{Cr} 12 \mathrm{Vol}$ \% MWCNT composite sintered at $1050{ }^{\circ} \mathrm{C}$. 


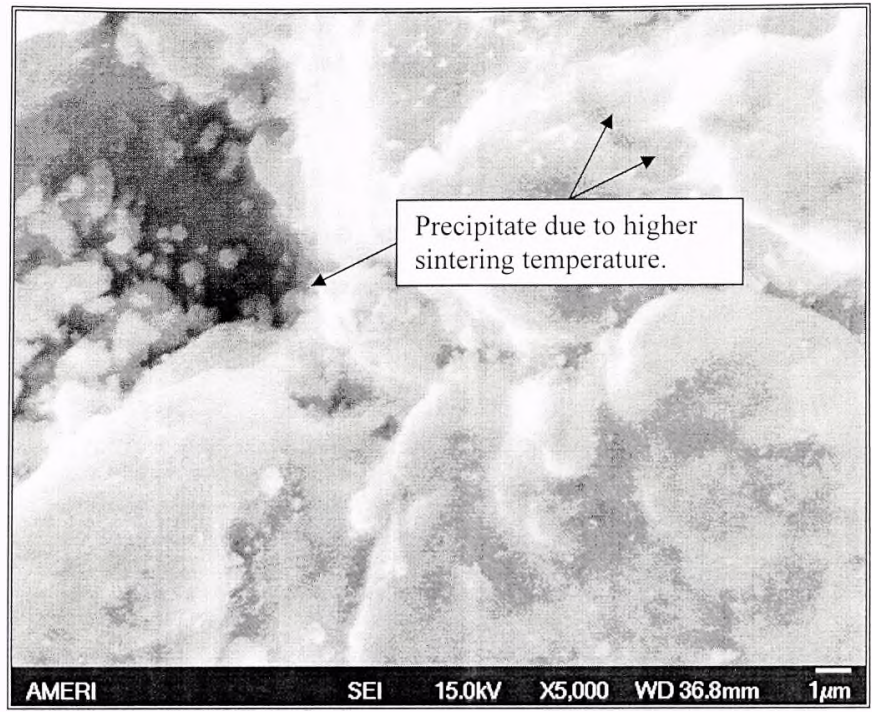

Figure 9.35: SEM images of $\mathrm{Cu}-\mathrm{Cr} 14 \mathrm{Vol} . \% \mathrm{MWCNT}$ composite sintered at $1050{ }^{\circ} \mathrm{C}$.
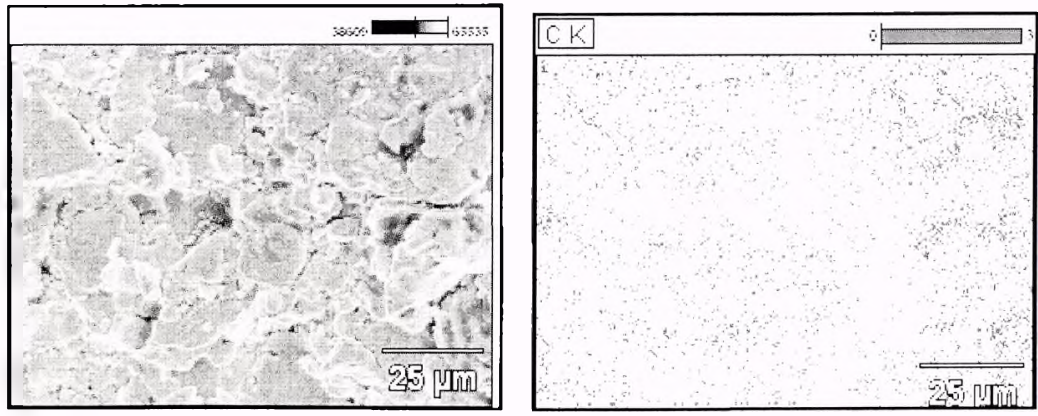

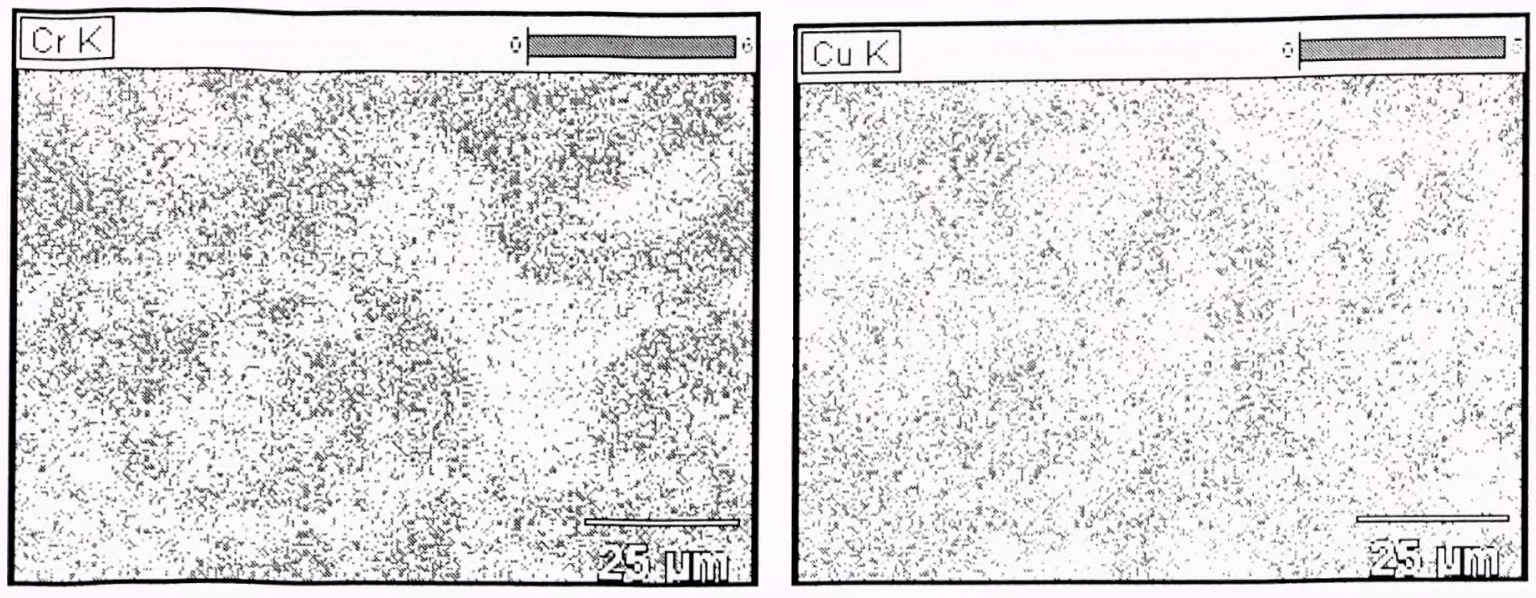

Figure 9.36: Mapping results of Cu-Cr $14 \mathrm{Vol} \%$ MWCNT composite sintered at $1050^{\circ} \mathrm{C}$.

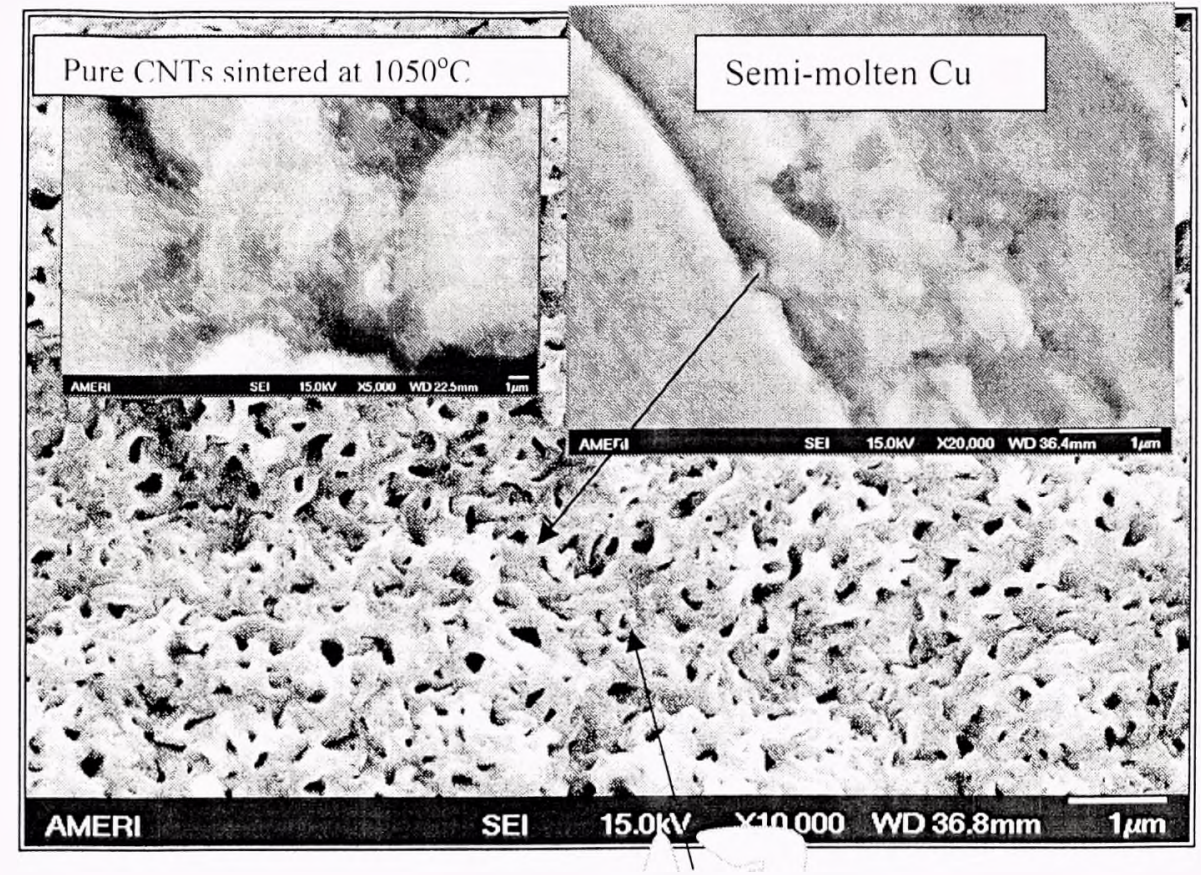

CNTs \& Cr coated with semi-molten $\mathrm{Cu}$

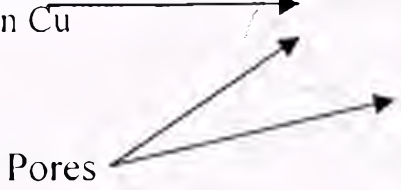

Figure 9.37: SEM image of Cu-Cr 16 Vol. \% MWCNT composite sintered at $1050{ }^{\circ} \mathrm{C}$.

In the composite sintered at $1050^{\circ} \mathrm{C}, \mathrm{Cu}$ particles were in the semi molten stage. As previously discussed, the precipitation of $\mathrm{Cr}$ from the $\mathrm{C} u$ matrix resulted in an 
increase in porosity. The photomicrograph in Figure 9.37 at X-20,000 magnification shows, the highly porous composite.
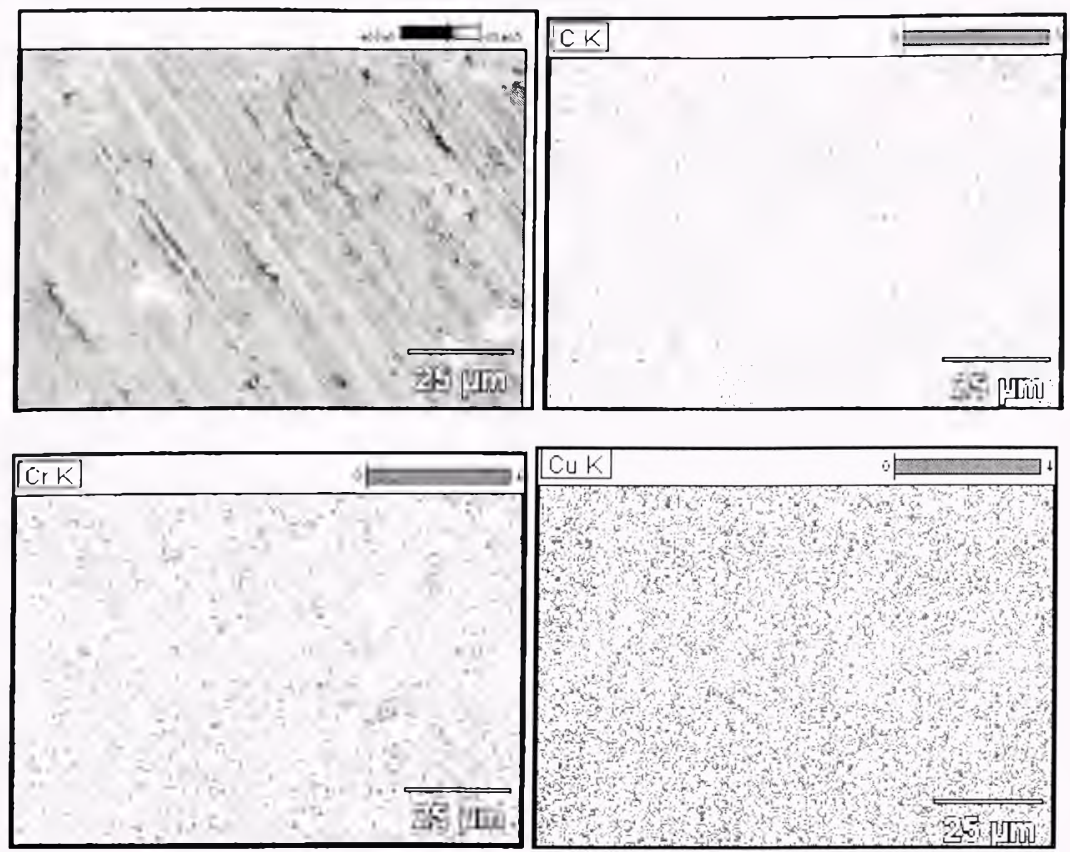

Figure 9.38: Mapping results of $\mathrm{Cu}-\mathrm{Cr} 16 \mathrm{Vol}$ \% MWCNT composite showing homogeneous distribution.

It is noticed above that the amount of CNTs found in the Cu-Cr 16 Vol.\% MWCNT composite was low. This could be attributed to the CNTs being covered by molten $\mathrm{Cu}$ and/or some formation of chromium carbide. It was also observed that $\mathrm{Cr}$ was more prevalent in the elemental mapping analysis although the percentage of $\mathrm{Cr}$ added to the composite was very small. Hence, this supports the premise that $\mathrm{Cr}$ precipitation occurred in composites sintered at $1050^{\circ} \mathrm{C}$. 


\subsection{TEM Analysis of Cu-Cr 10 Vol. \% MWCNT Composite}

The TEM micrograph of Cu-Cr $10 \mathrm{Vol} . \% \mathrm{MWCNT}$ composite sintered at $750^{\circ} \mathrm{C}$ and $900{ }^{\circ} \mathrm{C}$ (Figure 9.39 and 9.40 ) revealed agglomeration of MWCNTs. Also, MWCNTs produce bonding mechanisms, which may be described as rope anchoring, rebar reinforcing and bridging with the $\mathrm{Cu}-\mathrm{Cr}$ matrix. Based upon the analyses of TEM photomicrographs, the composite may also be described as having a bi-modal morphology as depicted in Figures 9.39 and 9.40 where the white region was composed of $\mathrm{Cu}-\mathrm{Cr}$ and the darker region at the periphery of the white region was composed of MWCNT-Cr. The bridging and rope anchoring of MWCNTs were observed between CuCr regions.

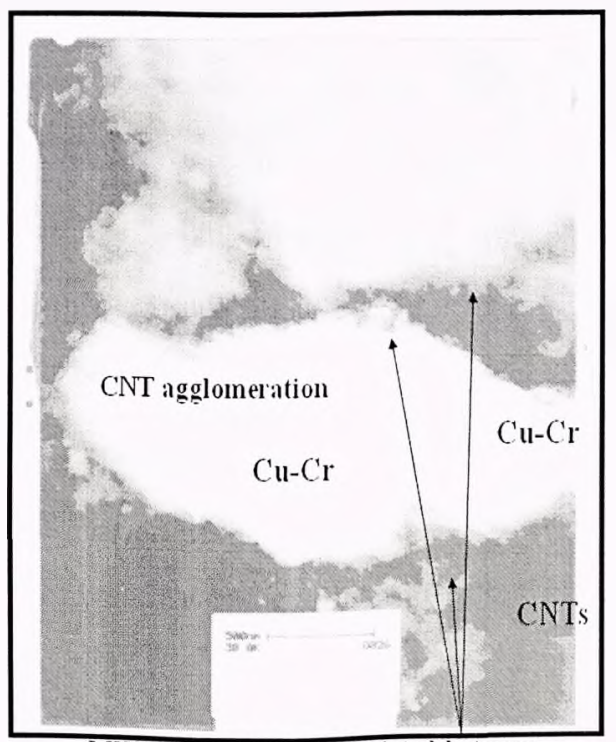

MWCNTs bridging is depicted between the Cu-Cr matrix

Figure 9.39: TEM photomicrograph of $\mathrm{Cu}-\mathrm{Cr} 10 \mathrm{Vol}$. \% MWCNT composite sintered at $750^{\circ} \mathrm{C}$. 


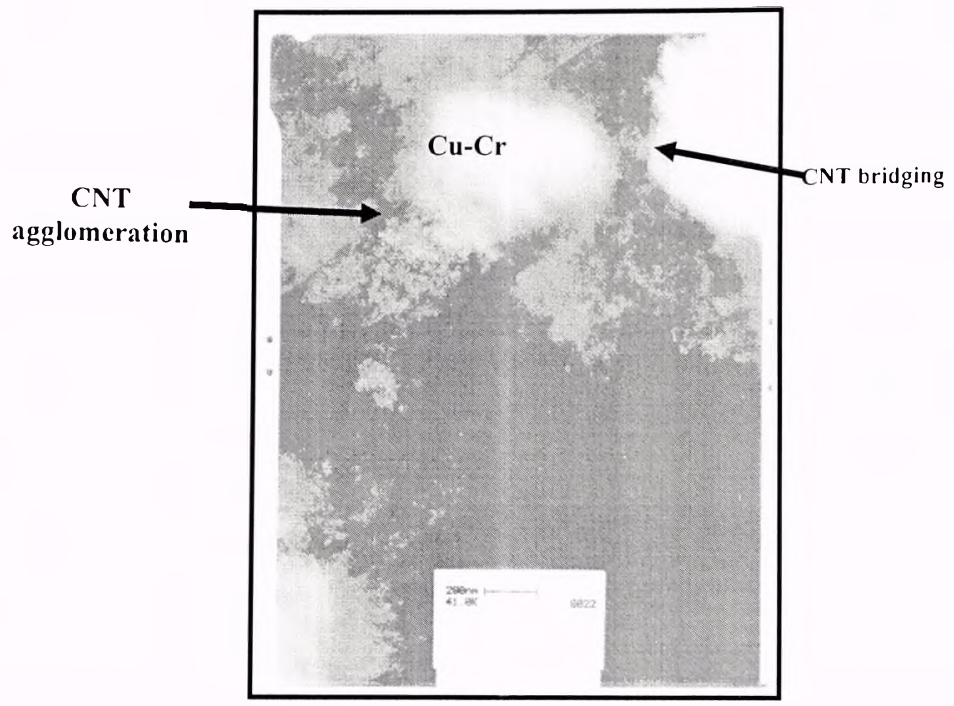

Figure 9.40: TEM photomicrograph of $\mathrm{Cu}-\mathrm{Cr} 10 \mathrm{Vol}$ \% MWCNT composite sintered at $900{ }^{\circ} \mathrm{C}$. 


\section{DENSITY}

\subsection{Variation of density with CNT volume content}

Feng $\mathrm{Yi}$ et. al. (2005) described the variation in density of the Ag-matrix composite reinforced by CNT. Figure 10.1, shows that, when the CNT volume content was less than $8 \%$, the relative density of the composites increased with CNT content once the volume content was greater than $10 \%$, the relative density decreased but rapidly.

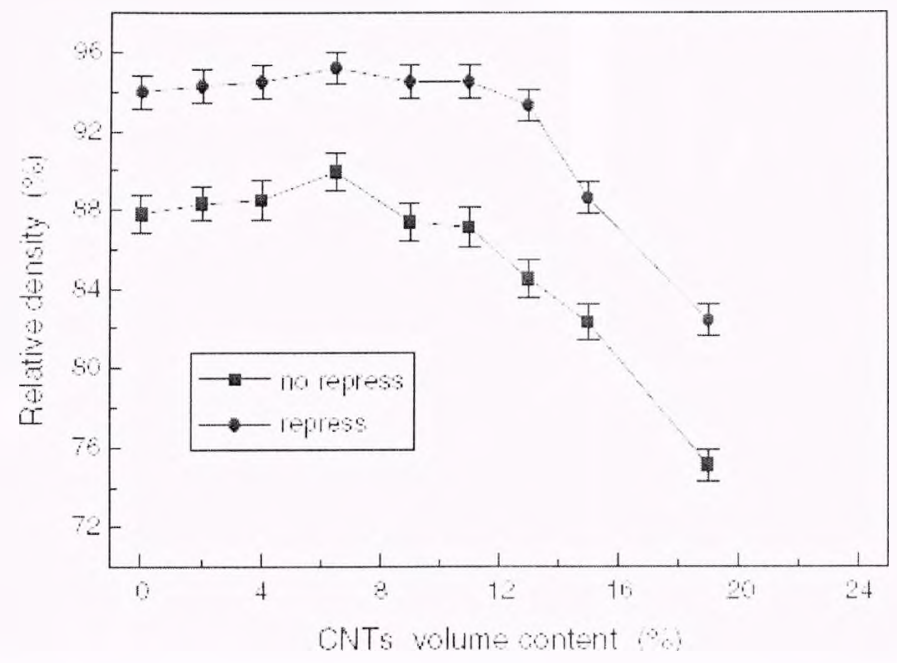

Figure 10.1: Variation of composite relative density with CNT volume content [76].

\subsection{Density measurements}

The density of the sintered and unsintered pellets was calculated from the Archimedes Principle in order assess the degree of compactness of the composite. The method for calculating the density was discussed in previous section. The density of the green compact obtained depended mainly on particle sire, which influenced packing 
density, and plasticity of the powders. The table below shows the density measurements of the pure Cu plate (C26000), as calculated and compared with the reference.

Table 10.1: Density measure for pure Cu sample.

\begin{tabular}{|c|c|}
\hline Sample & Density $\mathbf{g} / \mathbf{c m}^{\mathbf{3}}$ \\
\hline Cu alloy (C26000) & 8.5 \\
\hline Cu alloy (C26000) [74] & 8.53 \\
\hline
\end{tabular}

Table 10.2: Density of Green pellet at three different sintering temperatures.

\begin{tabular}{|c|c|c|c|c|}
\hline \multirow[b]{2}{*}{ Sample } & \multirow[b]{2}{*}{$\begin{array}{c}\text { Green pcllet } \\
\left(\mathrm{g} / \mathrm{cm}^{3}\right)\end{array}$} & \multicolumn{3}{|c|}{ Sintering Temperature (Density) } \\
\hline & & $\begin{array}{l}750^{\circ} \mathrm{C} \\
\left(\mathrm{g} / \mathrm{cm}^{3}\right)\end{array}$ & $\begin{array}{l}900^{\circ} \mathrm{C} \\
\left(\mathrm{g} / \mathrm{cm}^{3}\right)\end{array}$ & $\begin{array}{l}1050^{\prime \prime} \mathrm{C} \\
\left(\mathrm{g} / \mathrm{cm}^{3}\right)\end{array}$ \\
\hline Cu-Crl5 wt. $\%$ & 7.7470 & 7.7908 & 8.1870 & 7.5038 \\
\hline Cu-Cr 8 Vol.\% MWCNT & 6.9119 & 7.3894 & 7.4928 & 7.1006 \\
\hline $\mathrm{Cu}-\mathrm{Cr} 10 \mathrm{Vol} \% \mathrm{MWCNT}$ & 6.9432 & 7.5030 & 8.7839 & 8.6263 \\
\hline $\mathrm{Cu}-\mathrm{Cr} 12 \mathrm{Vol} . \% \mathrm{MWCNT}$ & 6.85006 & 7.4156 & 8.1048 & 7.2906 \\
\hline Cu-Cr 14 Vol.\% MWCNT & 6.7679 & 7.1667 & 7.4101 & 6.8601 \\
\hline Cu-Cr 16 Vol. $\%$ MWCNT & 6.4252 & 6.7440 & 6.8655 & 6.5139 \\
\hline
\end{tabular}

The densities of the $\mathrm{Cu}-\mathrm{Cr}$ alloy and $\mathrm{Cu}-\mathrm{Cr}-\mathrm{MWCNT}$ composites at three different sintering temperatures (i.e. $750{ }^{\circ} \mathrm{C}, 900^{\circ} \mathrm{C}$ and $1050^{\circ} \mathrm{C}$ ) were also determined. 
There was a large variation in the density values obtained at varying composition and sintering temperature. Shurong and Xiaobin (1999), discussed how the content of the CNTs in Cu-based composites has a significant effect on its degree of compactness [31].

In this investigation, it was observed that the density of the composite increased upto a CNT content of $10 \mathrm{Vol} . \%$ and then it decreased between $\mathrm{Cu}$ and $\mathrm{Cr}$ particles. The reason for an increase in density is that small particles of CNTs filled the pores, thus resulting in increased density. However, after 10 Vol. \% CNTs, the density decreased, due to the fact that all void spaces become filled, and additional CNTs begin to agglomerate, which leads to a reduction of the compactness.

The schematic diagrams depicted in Figure 10.3 illustrate the effect of addition of CNT's to $\mathrm{Cu}$ and $\mathrm{Cr}$ particles. At $10 \mathrm{Vol}$ \% MWCNTs all void spaces in the $\mathrm{Cu}-\mathrm{Cr}$ composites are filled. However, from 12 Vol. \% CNTs, addition CNI's tend to agglomerate. 

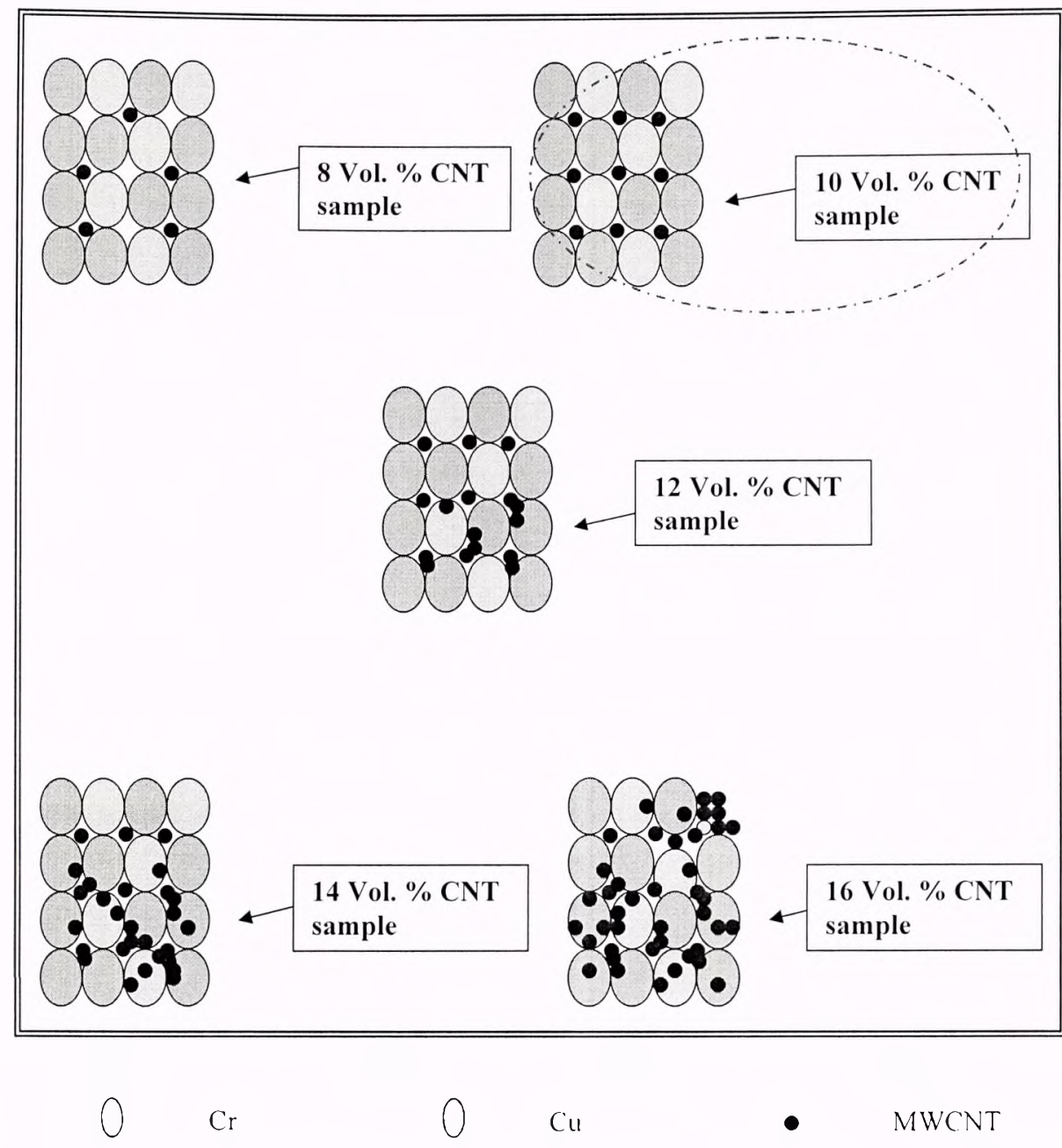

Figure 10.2: Schematic of packing arrangement of CNTs, Cu and Cr particles in the Cu-

$$
\text { Cr-MWCNT composite. }
$$

Figure 10.3 shows the variation of the density of unsintered and sintered composites as a function of temperature and CNT content. 


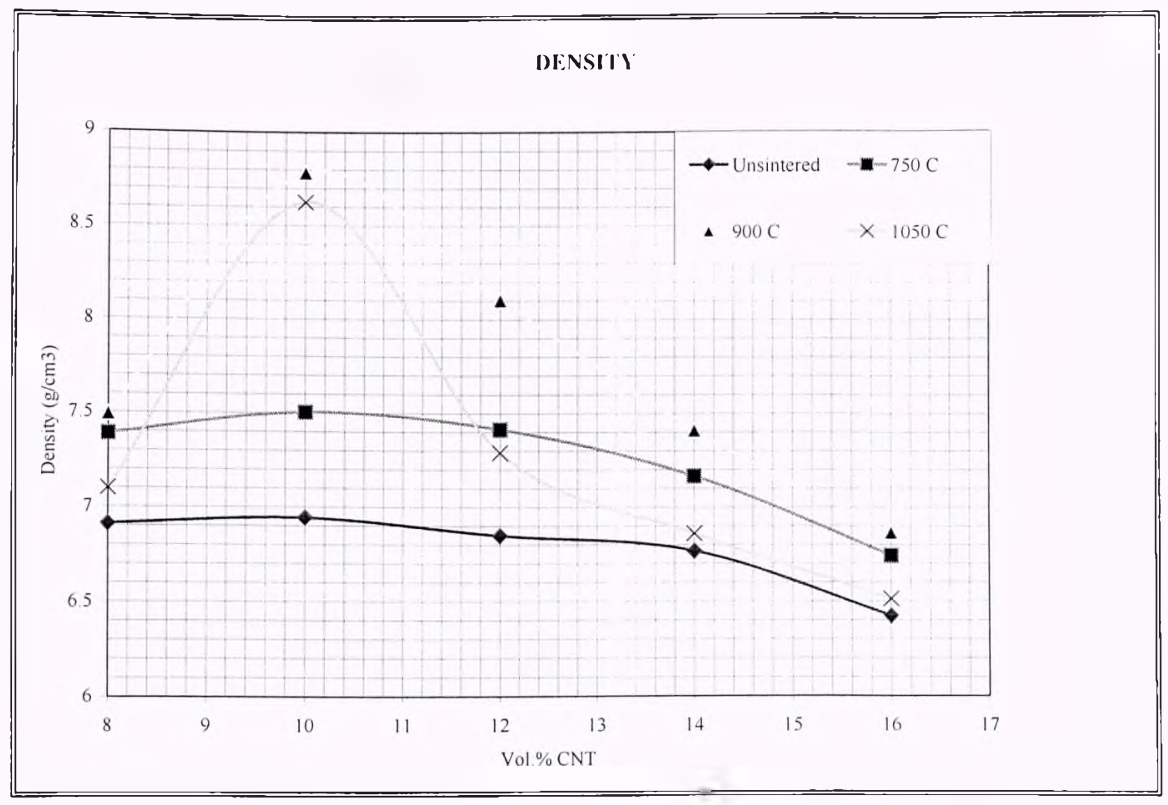

Figure 10.3: Density versus Vol. \% MWCNT plot.

\subsection{Linear and Volume Shrinkage}

The volume and linear shrinkage of the compacts were determined by using the relation:

$$
\begin{aligned}
& \text { Volume shrinkage }=\frac{\rho_{\text {num }}}{\rho_{\text {sinkerel }}} \\
& \text { Linear shrinkage }\left(\frac{\rho_{\text {srous }}}{\rho_{\text {smined }}}\right)^{\prime}
\end{aligned}
$$

The density values were substituted in the aforementioned formulas. The results of the obtained volume and linear shrinkages are tabulated in Table 10.3 and 10.4 . 
Table 10.3: Volume shrinkage with variation of composition and sintering temperatures.

\begin{tabular}{|c|c|c|c|}
\hline \multicolumn{1}{|c|}{ Sample } & \multicolumn{2}{|c|}{ Sintering Temperature (Volume shrinkage) } \\
\cline { 2 - 4 } & $\mathbf{7 5 0}{ }^{\circ} \mathbf{C}$ & $\mathbf{9 0 0}{ }^{\circ} \mathrm{C}$ & $\mathbf{1 0 5 0}{ }^{\circ} \mathbf{C}$ \\
\hline Cu-Cr15 wt.\% & 0.9944 & 0.9463 & 1.0324 \\
\hline Cu-Cr 8 Vol.\% MWCNT & 0.9354 & 0.9225 & 0.9735 \\
\hline Cu-Cr 10 Vol.\% MWCNT & 0.9254 & 0.7905 & 0.8049 \\
\hline Cu-Cr 12 Vol.\% MWCNT & 0.9237 & 0.8452 & 0.9396 \\
\hline Cu-Cr 14 Vol.\% MWCNT & 0.9444 & 0.9133 & 0.9866 \\
\hline Cu-Cr 16 Vol.\% MWCNT & 0.9527 & 0.9359 & 0.9864 \\
\hline
\end{tabular}

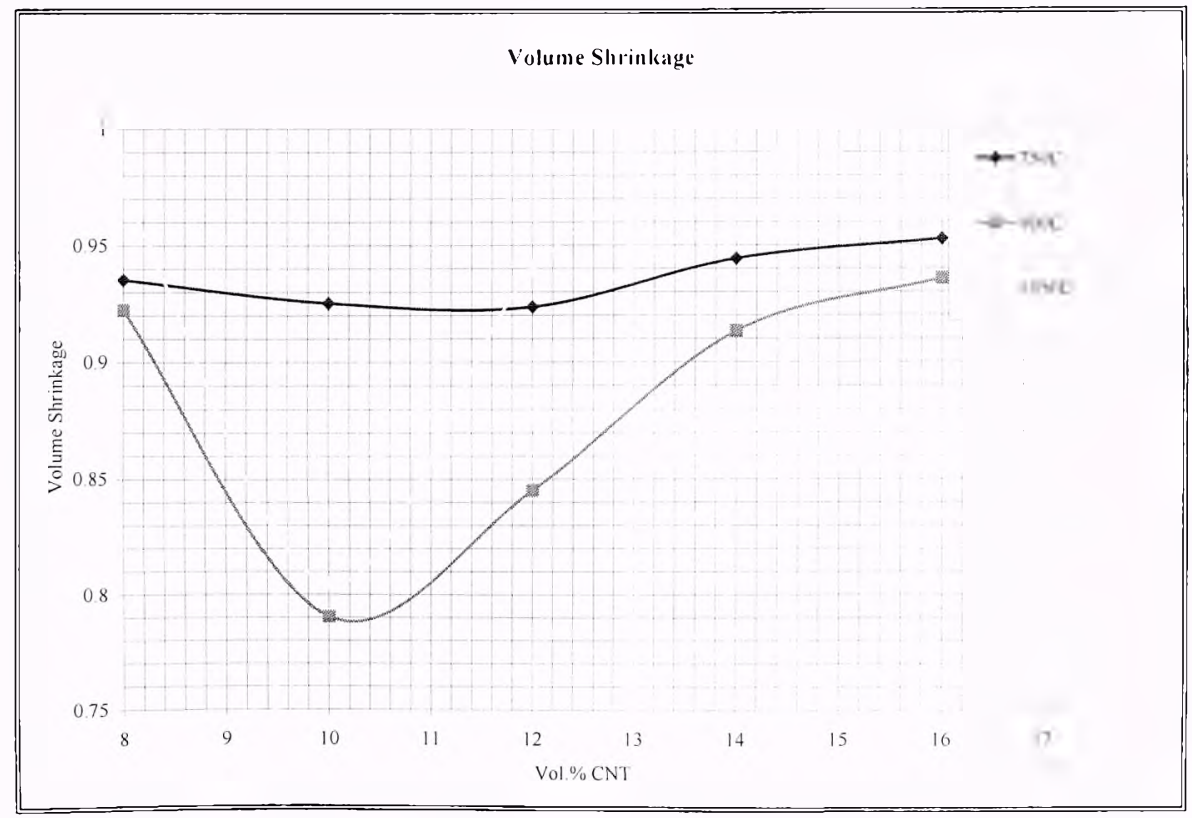

Figure 10.4: Volume shrinkage Vs Vol. \% MWCNT. 
Table 10.4: Linear shrinkage variations at different sintering temperatures.

\begin{tabular}{|c|c|c|c|}
\hline \multirow[t]{2}{*}{ Sample } & \multicolumn{3}{|c|}{ Sintering Temperature (Linear shrinkage) } \\
\hline & $750^{\circ} \mathrm{C}$ & $900^{\circ} \bar{C}$ & $1050)^{\circ} \mathrm{C}$ \\
\hline $\mathrm{Cu}-\mathrm{Cr} 15$ wt. $\%$ & 0.9981 & 0.9818 & 1.0107 \\
\hline Cu-Cr 8 Vol.\% MWCNT & 0.9780 & 0.9735 & 0.9911 \\
\hline Cu-Cr 10 Vol.\% MWCNT & 0.9745 & 0.9246 & 0.9303 \\
\hline Cu-Cr 12 Vol.\% MWCNT & 0.9739 & 0.9455 & 0.9794 \\
\hline Cu-Cr 14 Vol.\% MWCNT & 0.9811 & 0.9702 & 0.9955 \\
\hline Cu-Cr 16 Vol.\% MWCNT & 0.9840 & 0.9782 & 0.9954 \\
\hline
\end{tabular}

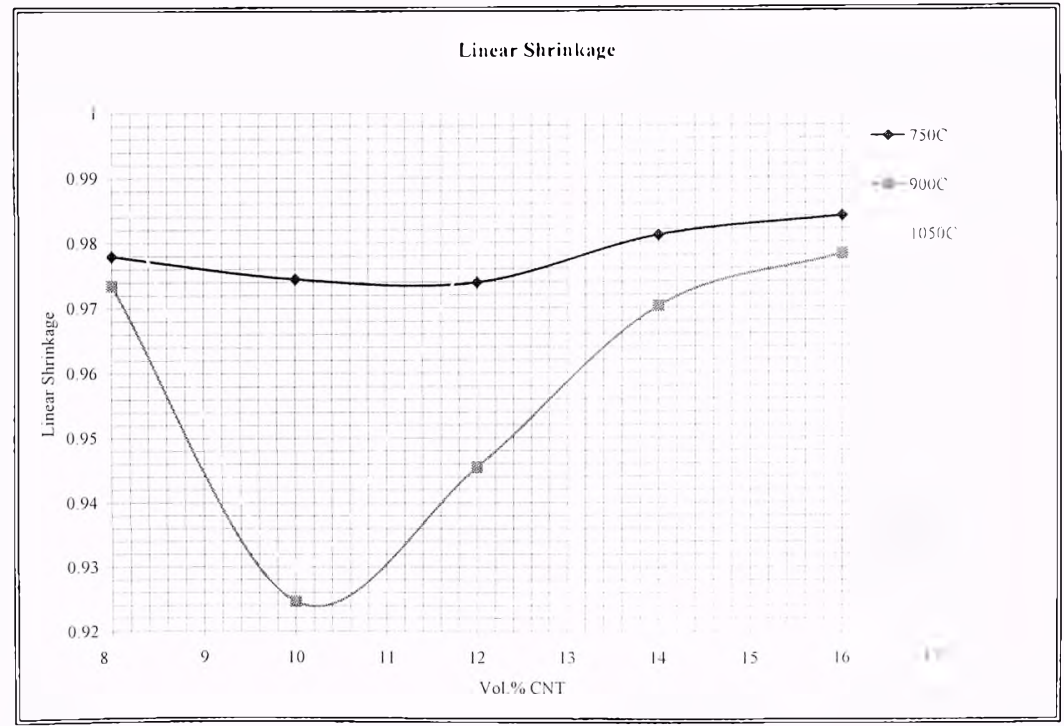

Figure 10.5: Linear shrinkage Vs Vol. \% MWCNT. 
Figure 10.4 and 10.5 , revealed that there was less shrinkage at $\mathrm{Cu}-\mathrm{Cr}-10 \mathrm{Vol} . \%$ MWCNT because the percentage addition of MWCNTs to the composite is the optimum amount to fill in the voids as discussed in section 10.2. 


\section{CONCLUSIONS}

- The MWCNTs were successfully incorporated in the Cu-Cr composite using a powder metallurgy technique.

- The 10 Vol. \% MWCNT composite (sintered at $900^{~ " C)}$ ) possessed high Vicker's hardness of $145 \mathrm{VH}$ as compared with that of $\mathrm{Cu}-\mathrm{CNT}(118 \mathrm{VH})$

- The composite with $10 \mathrm{Vol}$. \% MWCNT (sintered at $900{ }^{\circ} \mathrm{C}$ ) achieved an electrical conductivity $(1.11 \mathrm{E}+09 \mathrm{~S} / \mathrm{m}) 18$ times greater than that of pure copper $(5.96 \mathrm{E}+07 \mathrm{~S} / \mathrm{m})$.

- TEM/SEM photomicrographs revealed the presence of MWCNT, which acted as rope anchors and bridges with the $\mathrm{Cu}-\mathrm{Cr}$ matrix, confirming minimal damage.

- The Cu-Cr-MWCNT composite displayed a bi-modal morphology (Cu-Cr and MWCNT-Cr). 


\section{FUTURE WORK}

- Decrease the porosity of the composite using Hot Isostatic Pressing (HIP).

- Measure mechanical properties (ductility, strength etc.) of the composite.

- Perform aging to determine its effect on electrical conductivity and porosity.

- Determine the optimal sintering temperature for cost effectiveness. 


\section{REFERENCES}

[1] Baihe Miano, Yan Zhang and Guoxun Liu, "Current Status and developing Trends of $\mathrm{Cu}-\mathrm{Cr}$ Contact Materials for VCB". University of Science and Technology Beijing, Beijing 100083, China, (2004).

[2] Chitoshi Masuda and Yoshihisa Tanaka, "Fatigue properties of $\mathrm{Cu}-\mathrm{Cr}$ in situ composites". International Journal of Fatigue, Volume 28, Issue 10, (2006), pg. 1426-1434.

[3] A. Peigney, E. Flahaut, Ch. Laurent, F. Chastel and A. Rousset, "Aligned carbon nanotubes in ceramic-matrix nanocomposites prepared by hightemperature extrusion". CIRIMAT, UMR CNRS 5085/LCMIE, Centre Interuniversitaire de Recherche et d'Ingenierie des Materiaux, Université PaulSabatier, Toulouse cedex 4, France, (2001).

[4] M. M. J. Treacy, T.W. Ebbesen and J.M. Gibson (1996) Exceptionally high Young's modulus observed for individual carbon nanotubes. Nature, 381, 678680.

[5] Z. L. Wang, R.P. Gao, P. Poncharal, W.A. de Heer, Z.R. Dai and Z.W. Pan (2001) Mechanical and electrostatic properties of carbon nanotubes and nanowires. Mat. Sc. Eng. C, 16, 3-10.

[6] S. Iijima (1991) Helical microtubules of graphitic carbon. Nature 354, 56-58.

[7] W.A. Heer, A. Chatelain and D.A. Ugarte (1995) Carbon nanotube fieldemission electron source, Science 270, 1179-1180.

[8] R.F. Service (1996) Mixing nanotube structures to make a tiny switch, Science $271,1232-1233$

[9] J. Jang, J. Bae and S.H. Yoon (2003) A study on the effect of surface treatment of carbon nanotubes for liquid crystalline epoxide-carbon nanotube composite. J. Mater. Chem. 13, 676-681.

[10] J. Liu, M. Shao, X. Chen, W. Yu, X. Liu and Y. Qian (2003) Large-scale synthesis of carbon nanotubes by an ethanol thermal reduction process, J. Am. Chem. Soc. 125 (27), 8088-8089.

[11] P.G. Collins and P. Avouris (2000) Nanotubes for electronics, Sci. Am. 283, 62-69.

[12] D. Raabe, K. Miyake and H. Takahara, "Processing, microstructure, and properties of ternary high-strength $\mathrm{Cu}-\mathrm{Cr}-\mathrm{Ag}$ in situ composites". Max-PlanckInstitut für Eisenforschung, Max-Planck-Str. 1, 40237 Dusseldorf, Germany, 
Corporate Research and Development Center, Mitsui Kinzoku, pg. 1333-2, Saitama 362, Japan, (2000).

[13] A. Peigney, E. Flahaut, Ch. Laurent, F. Chastel and A. Rousset, "Aligned carbon nanotubes in ceramic-matrix nanocomposites prepared by hightemperature extrusion". Cirimat, Umr Cnrs 5085/lcmie, Centre Interuniversitaire de Recherche et d'Ingénierie des Matériaux, Université PaulSabatier, Toulouse cedex 4, France, (2001).

[14] Lijie Ci, Zhenyu Ryu, Neng Yun Jin-Phillipp and Manfred Rühle, "Investigation of the interfacial reaction between multi-walled carbon nanotubes and aluminum". Max-Planck-Institute for Metals Research, Heisenbergstr. 3, D70569 Stuttgart, Germany, (2006).

[15] X.L. Shi, H. Yang, G.Q. Shao, X.L. Duan, L. Yan, Z. Xiong and P. Sun, "Fabrication and properties of W-Cu alloy reinforced by multi-walled carbon nanotubes". State Key Laboratories of Advanced Technology for Materials Synthesis \& Processing, Wuhan University of Technology, China, (2006).

[16] Feng Yi, Min Zhang and Yi Xu, "Effect of the electric current on the friction and wear properties of the CNT-Ag-G composites Carbon, Volume 43, Issue 13, (2005).

[17] O. Hjortstam, P. Isberg, S. Soderholm, and H. Dal, "Can we achieve ultralow resistivity in carbon nanotube-based metal composites?". Springer-Verlag, (2004).

[18] Christian P. deck, Kenneth Vecchio, "Prediction of carbon nanotube growth success by the analysis of carbon-catalyst binary phase diagrams". Material Science and Engineering Program, University of California, San Diego, La Jolla, CA 92093-0411, USA, (2005).

[19] Li H.J., Lu W. G., Li J. J., Bai X. D., and Gu C. Z., "Multichannel Ballistic Transport in Multiwall Carbon Nanotubes". Beijing National Laboratory for Condensed Matter Physics, Institute of Physics, Chinese Academy of Sciences, Beijing 100080, China, (2005).

[20] Mylvaganam Kausala and Zhang Liangchi C., "Fabrication and Application of Polymer Composites Comprising Carbon Nanotubes". Centre for Advanced Materials Technology, The University of Sydne, Australia, (2006).

[21] L.Q. Jiang and L. Gao (2003) Carbon nanotubes-magnetite nanocomposites from solvothermal processes: formation, characterization, and enhanced electrical properties, Chem. Mater. 15, 2848-2853. 
[22] T. Kuzumaki, K. Miyazawa, H. Ichinose, K. Ito (1998) Processing of carbon nanotube reinforced aluminum composite. J. Mater. Res. 13, 2445-9.

[23] C.L. Xu, B.Q.Wei, R.Z. Ma, J. Liang, X.K. Ma, D.H. Wu (1999) Fabrication of aluminium- carbon nanotube composites and their electrical properties. Carbon. 37, 855-8.

[24] R. George, K.T. Kashyap, R. Rahul, S. Yamdagni (2005) Scripta Mater. 53,1159-63.

[25] K. T. Lau (2003) Chem. Phys. Lett. 370, 399-405.

[26] A. Peigney (2003) Nat. Mater. 2, 15-16.

[27] E. Flahaut, A. Peigney, Ch. Laurent, Ch. Marliere, F. Chastel and A. Rousset (2000) Acta Mater. 48, 3803-12.

[28] X. Wang, N. P. Padture, H. Tanaka (2004) Nat. Mater. 3, 539-44.

[29] Cha S I, Kim K T, Arshad S N, Mo C B and Hong S H (2005) Adv. Mater. 17, 1377-81.

[30] Kim Kyung Tae, Cha Seung II, Hong Seong Hyeon, Hong Soon Hyung, "Microstructures and tensile behaviour of carbon nanotube reinforced $\mathrm{Cu}$ matrix nanocomposites". Department of Materials Science and Engineering, Korea Advanced Institute of science and Technology, and Nano P/M Group, Korea Institute of Machinery and Materials, (2006) .

[31] Shurong Dong and Xiaobin, "Mechanical properties of Cu-based composites reinforced by carbon nanotubes". Department of Information and Electronic Engineering and Department of Materials Science and Engineering, China, (1999).

[32] Esawi A. and Morsi K., "Dispersion of carbon nanotubes (CNTs) in aluminum powder". Department of Mechanical Engineering and The Science and Technology Research Center (SRTC), The American University in Cairo, Egypt and Department of Mechanical Engineering, San Diego State University, USA, (2006).

[33] Raabe D., Miyake K. and Takahara H., "Processing, microstructure, and properties of ternary high-strength $\mathrm{Cu}-\mathrm{Cr}-\mathrm{Ag}$ in situ composites". Max-Planck Institut fur Eisenforschung, Germany Corporate Research and Development Center, Mitsui Kinzoku, Japan, (2000).

[34] Dong S.R., Tu J.P. and Zhang X.B., "An investigation of the sliding wear behaviour of Cu-matrix composite reinforced by carbon nanotubes". 
Department of Information and Electronic Engineering, Department of materials Science and Engineering, Zhejiang University, China, (2001).

[35] Jiang IIongjin, Zhu Lingbo, Moon Kyoung-sik and Wong C.P., “ The preparation of stable metal nanoparticles on carbon nanotubes whose surfaces were modified during production". School of Materials Science and Engineering, School of Chemistry and Biochemistry and School of Chemical and Biomolecular Engineering, Georgia Institute of Technology, Atlanta, GA, USA, (2006).

[36] Curtin A. William and Sheldon W. Brian, "CNT-reinforced ceramics and metals". Division of Engineering, Brown University, Providence, USA, (2004).

[37] Laurent Ch., Peigney A., Dumortier O. and Rousset A., "Carbon NanotubesFe-Alumina Nanocomposites. Part II: Microstructure and Mechanical Properties of the Hot-Pressed Composite. Laboratoire de Chimie des Materiaux Inorganiques, University Paul Sabatier, France, (1997).

[38] Wu Shi-Hong, Masaharu Iwamoto, Natsuki Toshiaki, Ni Qing-Qing, "Electrical Conduction and Percolation Behaviour of Carbon nanotubes/UPR Nanocomposites". Division of Advanced Fibro-Science, Kyoto Institute of Technology, Faculty of Engineering, Shinshu University and Dept. of Functional Machienery and Mechanics, Japan, (2006).

[39] Kittel, Introduction to Solid State Physics, 7th Ed. Referenced to G. T. Meaden, Electrical resistance of metals, Plenum, 1965.

[40] How Alloying Elements Affect the Properties of Copper Alloys, MatWeb Material Property Data.

[41] J. S. Benjamin, Sci. Amer., 235, 5, 40-48, 1976.

[42] J. S. Benjamin, E. Arzt, L. Schultz, ed. New materials by mechanical alloying techniques. Oberursel, Germany: DGM Informationgesellschaft, 3-18, 1989.

[43] J. S. Benjamin, Metal Powder Rep., 45, 122-127, 1990.

[44] H. Zoz (1995) Attritor technology-latest developments, Mater. Sci. Forum. 179$181,419-424$.

[45] C. C Koch (1989) Materials synthesis by mechanical alloying, Annu. Rev. Mater. Sci. 18, 121-143.

[46] D. Basset, P. Matteazzi, and F. Mani (1993) Designing a high energy ball-mill for synthesis nanophase materials in large quantities, Mater. Sci. Eng. A168, 149-152. 
[47] J. S. Benjamin (1976) Sci. Am. 234. 40.

[48] B. S. Murthy, S. Ranganathan (1998) Int. Mater. Rev. 43. 101.

[49] C. Suryanarayana (2001) Progr. Mater. Sci. 46. 1.

[50] D. R. Maurice, T. H. Courtney, Metall. Trans., A21, 289-303, (1990).

[51] Suryanarayana C., "Mechanical Alloying and Milling". Technology \& Engineering, pp 36-37, 69, 399 and 400 (2004).

[52] Suryanarayana C., "Mechanical alloying and milling". Department of Metallurgical and Materials Engineering, Colorado School of Mines, USA, (2000).

[53] C. H. Lee, M. Mori, T. Fukunaga and U. Mizutani, "Effect of Ambient Temperature on the MA and MG Processes in Ni-Zr Alloy System". Department of Crystalline Materials Science, Nagoya University, (1990).

[54] Copper.org

[55] Suryanarayana $C^{a}$., Ivanov E." and Boldyrev V. V.c, "The science and technology of mechanical alloying". "The George S. Ansell Department of Metallurgical and Materials Engineering, Colorado School of Mines, 'Tosoh SMD, Inc., Grove City, OH, USA ${ }^{c}$ Institute of Solid State Chemistry and Mechanochemistry, Russian Academy of Sciences, Novosibirsk, Russia, (2001).

[56] F. Thummler and R.Oberacker, "An Introduction to Powder Metallurgy" The institute of Materials, London (1993).

[57] The Chemistry and Physics of Clays and Other Ceramic Materials, by Reff. W. Grimshaw. pp. 417-422, 441-442, (1971).

[58] Amruthaluri S., "Synthesis of Copper Carbon Nanotube Composite and its Electrical conductivity Measurement" Thesis, pp 20-22, (2008).

[59] Instron, The difference in measurability. Materials testing solutions. Website.

[60] T. Fetta,, A.B. Kounga Njiwab and J. Rödelb, "Crack opening displacements of Vickers indentation cracks" Forschungszentrum Karlsruhe, Institut fur Materialforschung II, Postfach 3640, D-76021 Karlsruhe, Germany and bDarmstadt University of Technology, Institute of Materials Science, Petersenstr. 23, 64287 Darmstadt, Germany, (2004).

[61] Gardiner, D.J. (1989). Practical Raman spectroscopy. Springer-Verlag. ISBN 978-0387502540. 
[62] Huang B. R., Huang C.S., Hsieh C.F. and Liu Y.F., "The electrical conduction mechanism of multiwalled carbon nanotubes film synthesized by microwave plasma chemical vapor deposition", Department of Electronic Engineering, Institute of Electronic Engineering, National Yunlin University of Science and Technology, 123, University Road, Section 3, Touliu Yunlin 640, Taiwan, R.O., (2004).

[63] Bendiab N., Anglaret E. and Bantignies J.-L., Phys. Rev., B 64 (2001), p. 245424.

[64] Jishi R.A., Venkataraman L., Dresselhaus M.S. and Dresselhaus G., Chem. Phys. Lett. 209 (1993), p. 77.

[65] Jishi R.A., Dresselhaus M.S. and Dresselhaus G., Phys. Rev., B 47 (1993), p. 16671 .

[66] Eklund P.C., Holden J.M. and Jishi R.A., Carbon 33 (1995), p. 959.

[67] Rao A.M., Richter E. and Bandow S., Science 275 (1997), p. 187.

[68] Christian P. deck and Kenneth Vecchio, "Prediction of carbon nanotube growth success by the analysis of carbon-catalyst binary phase diagrams". Material Science and Engineering Program, University of California, San Diego, La Jolla, CA 92093-0411, USA, (2005).

[69] Horst Kippenberg. Wilfried Kuhl and Wolfgang Schlenk, "Kontaktmaterial fur Vakuumschalter". Siemens Energie U. Automation, Vol. 7, pp. 18-21. 1985.

[70] Musso S., Giorcelli M., Pavese M., Bianco S., Rovere M., and Tagliaferro A., "Improving macroscopic physical and mechanical properties of thick layers of aligned multiwall carbon nanotubes by annealing treatment". Dipartimento di Fisica, Politecnico di Torino and Dipartimento di Scienza dei Materiali e Ingegneria Chimica, Politecnico di Torino, Torino, Italy, 2007.

[71] Rudy. E, "Compedium of Phase Diagram Data". Air Force Materials Laboratory Metals and Ceramics Division Wright-Patterson AFB, OHIO, Pg 179.

[72] Metals Handbook, Vol 8, Metallography, Structures and Phase Diagrams. Pg 290.

[73] Christian P. deck and Kenneth Vecchio, "Prediction of carbon nanotube growth success by the analysis of carbon-catalyst binary phase diagrams". Material Science and Engineering Program, University of California, San Diego, La Jolla, CA 92093-04I1, USA, (2005). 
[74] Callister William D., Jr. "Fundamentals of Materials Science and Engineering, An Integrated Approach, Second Edition, 2005, Pg-A4.

[75] Zhao Qing and Wagner H. Daniel., "Raman spectroscopy of carbonnanotube-based composites". Department of Materials and Interfaces, Weizmann Institute of Science, Rehovot 76100, Israel, (2004).

[76] Feng Yi, Yuan Hai Long and Zhang Min, "Fabrication and properties of silver-matrix composites reinforced by carbon nanotubes". Department of Materials Science and Engineering, Hefei University of Technology, Hefei 230009, People's Republic of China, (2005).

[77] Jin Y., Adachi K., Takeuchi T., and Suzuki H.G., "Correlation between the electrical conductivity and aging treatment for a $\mathrm{Cu}-15 \mathrm{wt} \% \mathrm{Cr}$ alloy composite formed in-situ". Mechanical Properties Diaision, National Research Institute for Metals, Tsukuba, Iburaki 305, Japan, (1997). 


\section{APPENDICES}

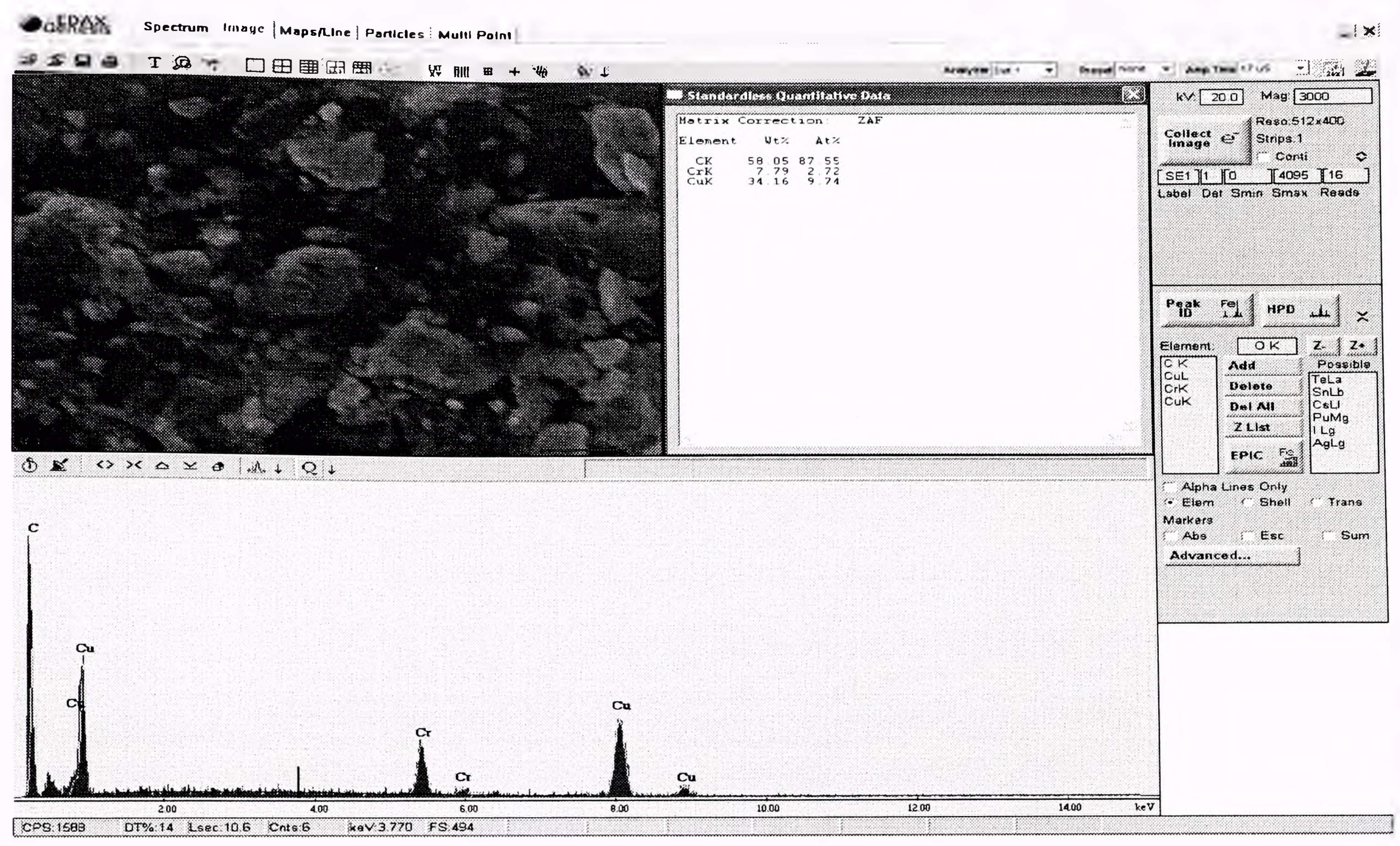

Figure A: EDS analysis of Cu-Cr 10 Vol. \% MWCNT.

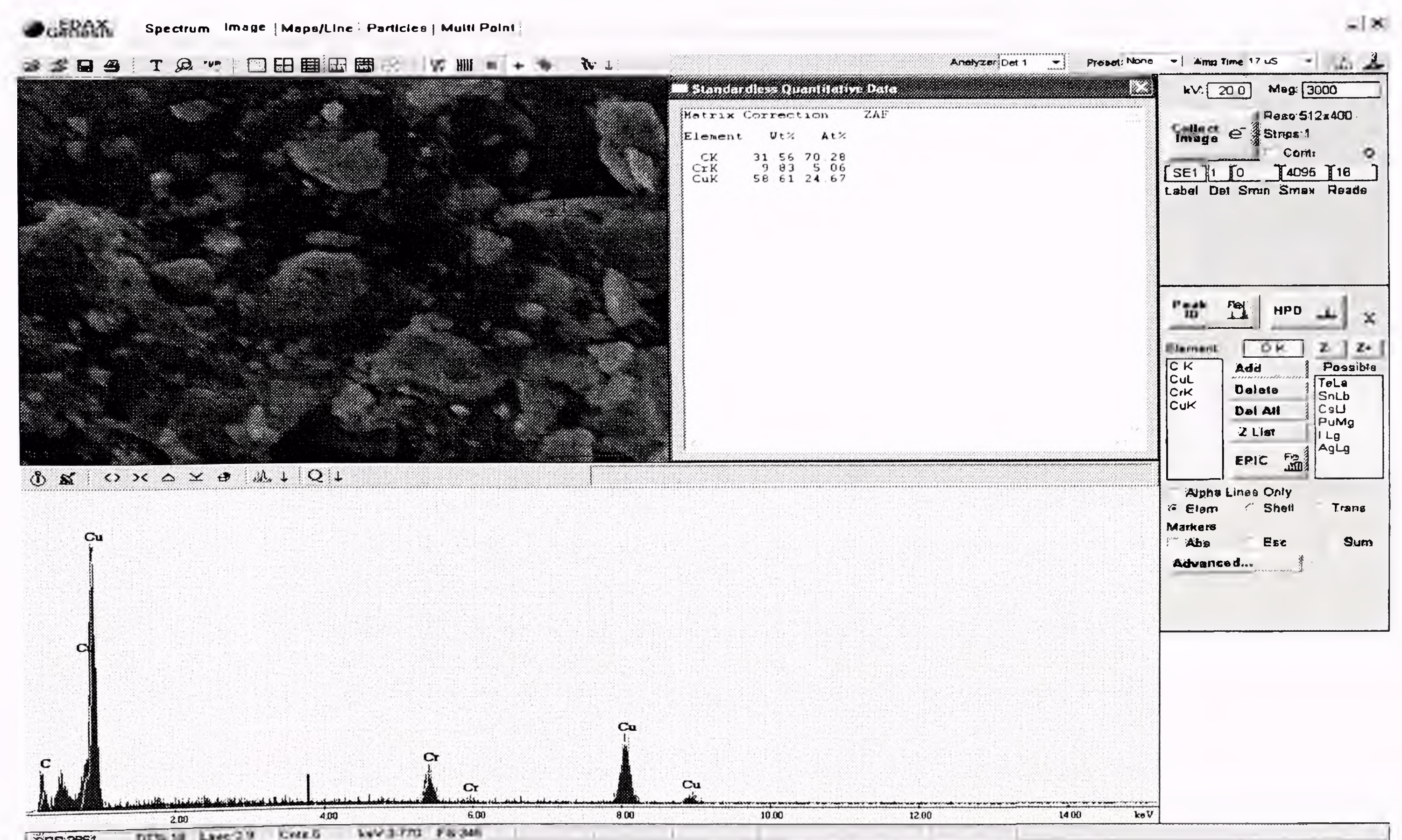




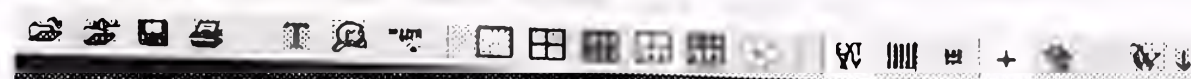

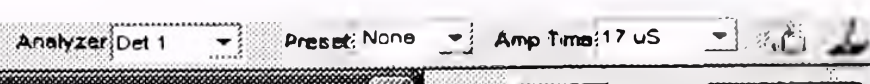
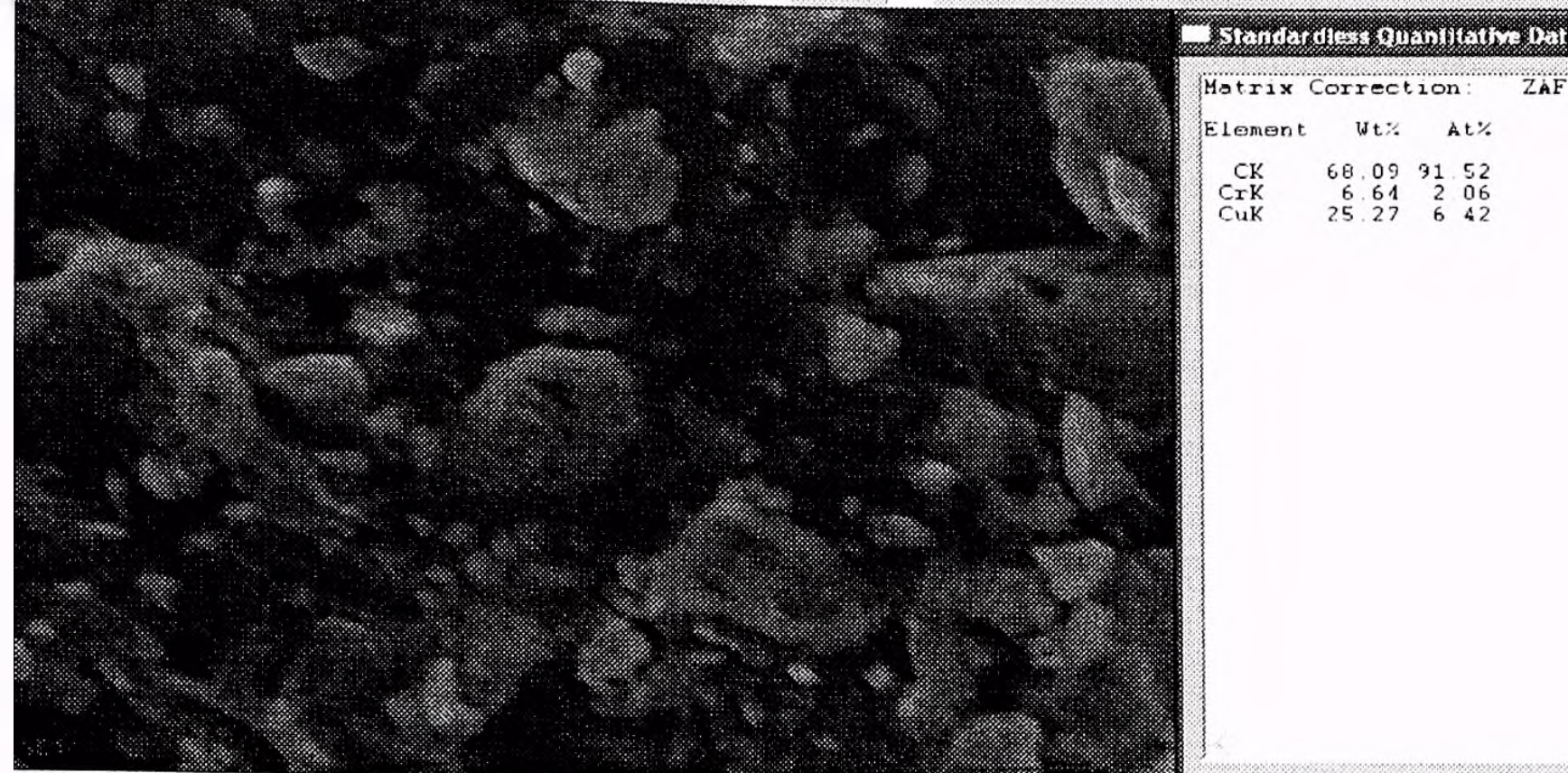

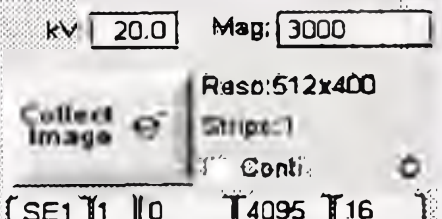

lement wt\% At\%

$\begin{array}{lllll}\mathrm{CK} & 68.09 & 91 & 52 \\ \mathrm{Cr} & 6.61 & 2 & 06 \\ \mathrm{CHK} & 25.27 & 6 & 42\end{array}$

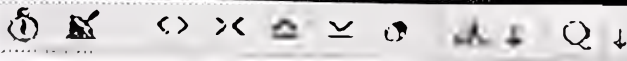

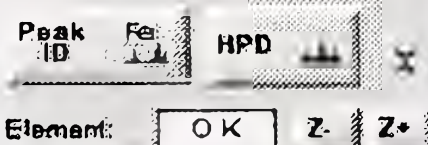

$C K$ Aad Possible

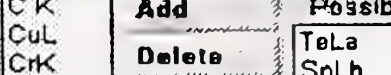

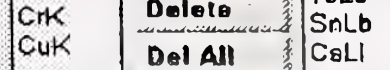

ZLiles EPIC Fe

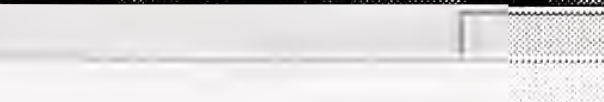

CPP:1620
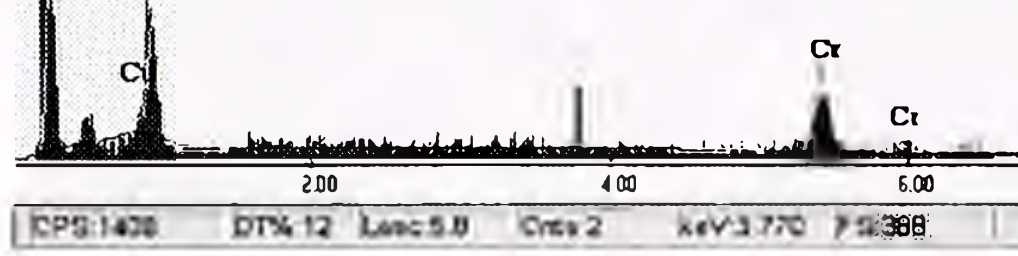

Cu

$10.00 \quad 1200 \quad 1400$ keV

Figure B: EDS analysis of $\mathrm{Cu}-\mathrm{Cr} 10 \mathrm{Vol}$ \% MWCNT.

- GBRAlls spectum linsye /Maps/Line / Paricles / Mull Point

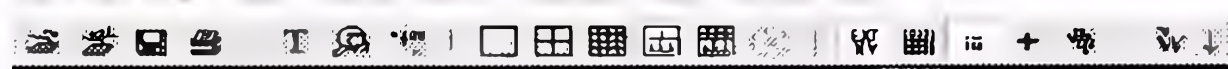

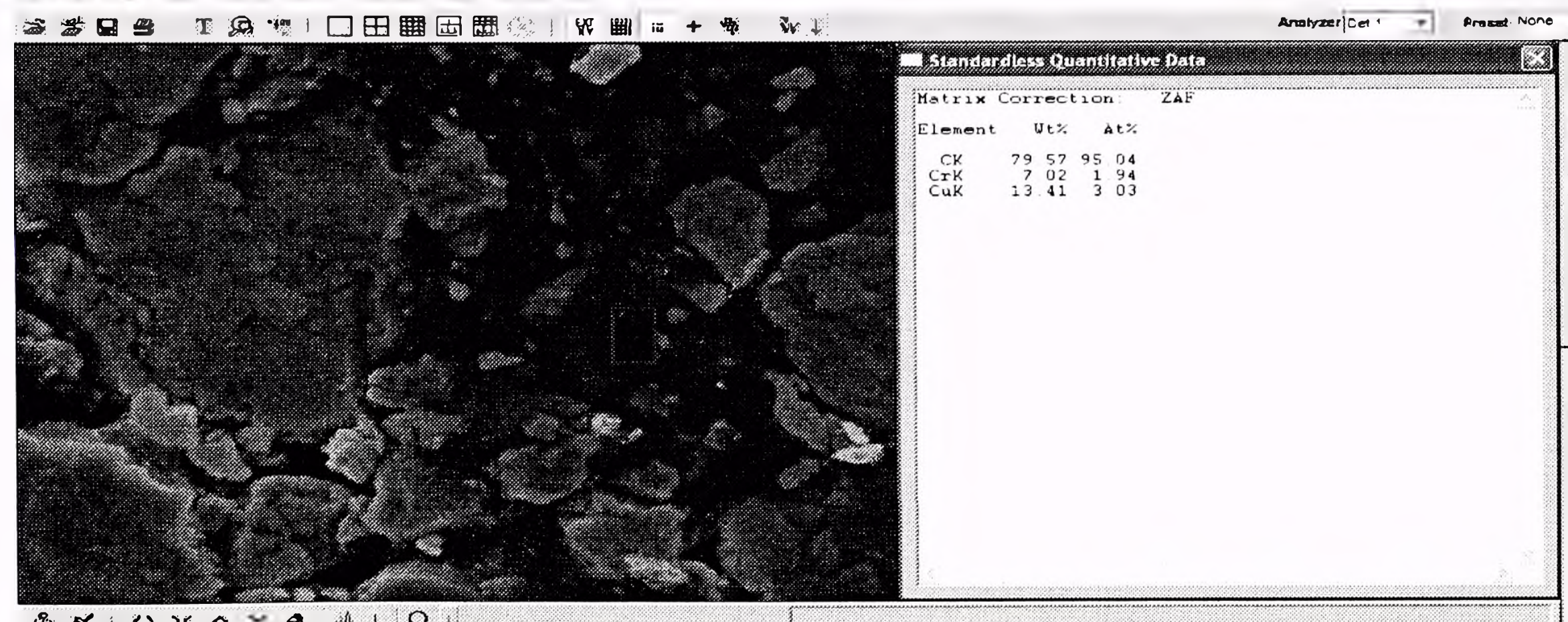

(9) $x: 23 \leqslant \leq x+2$.

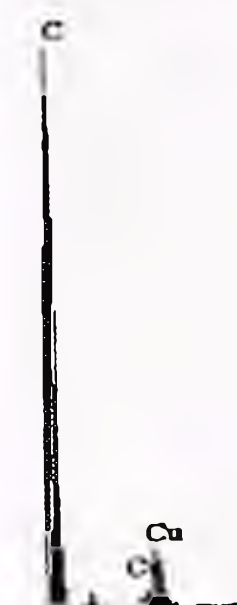

4.
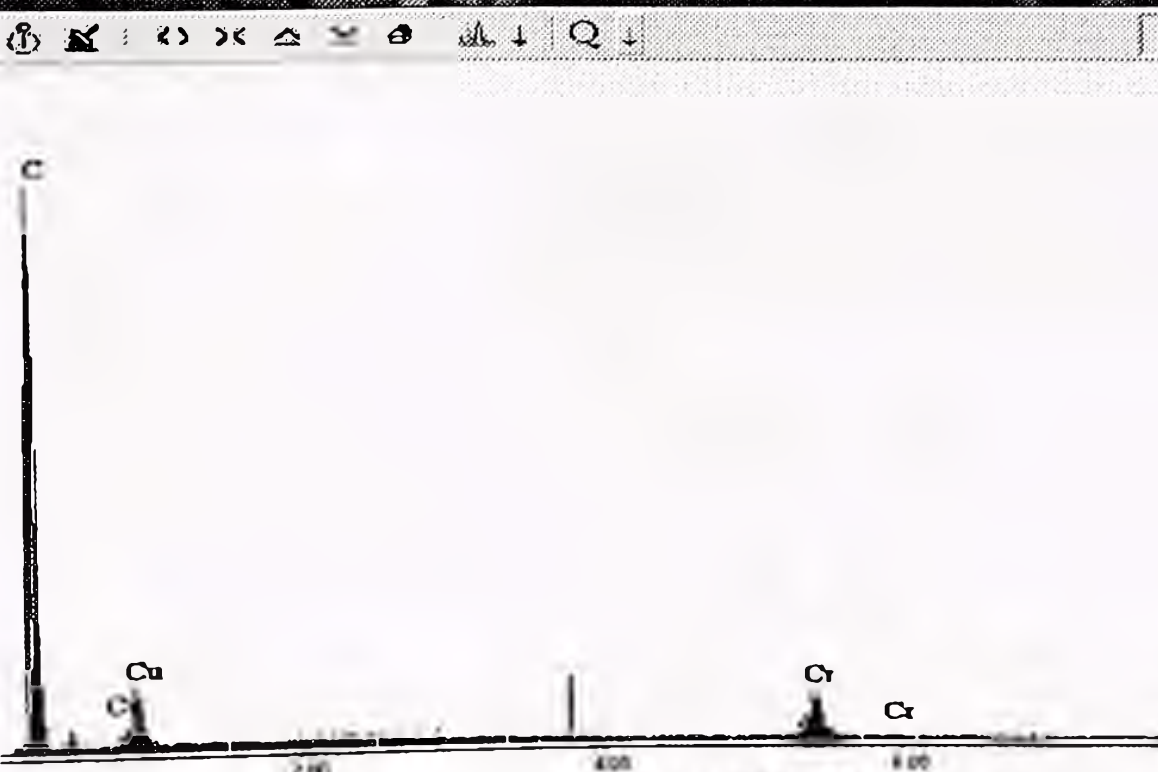

TPS 105
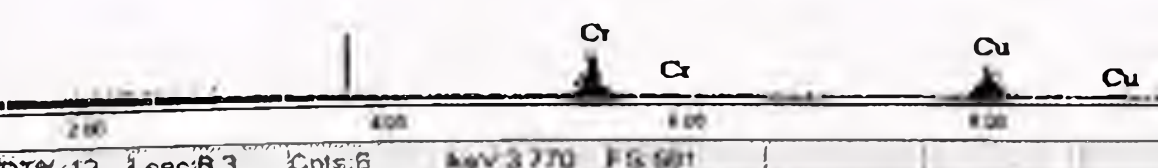


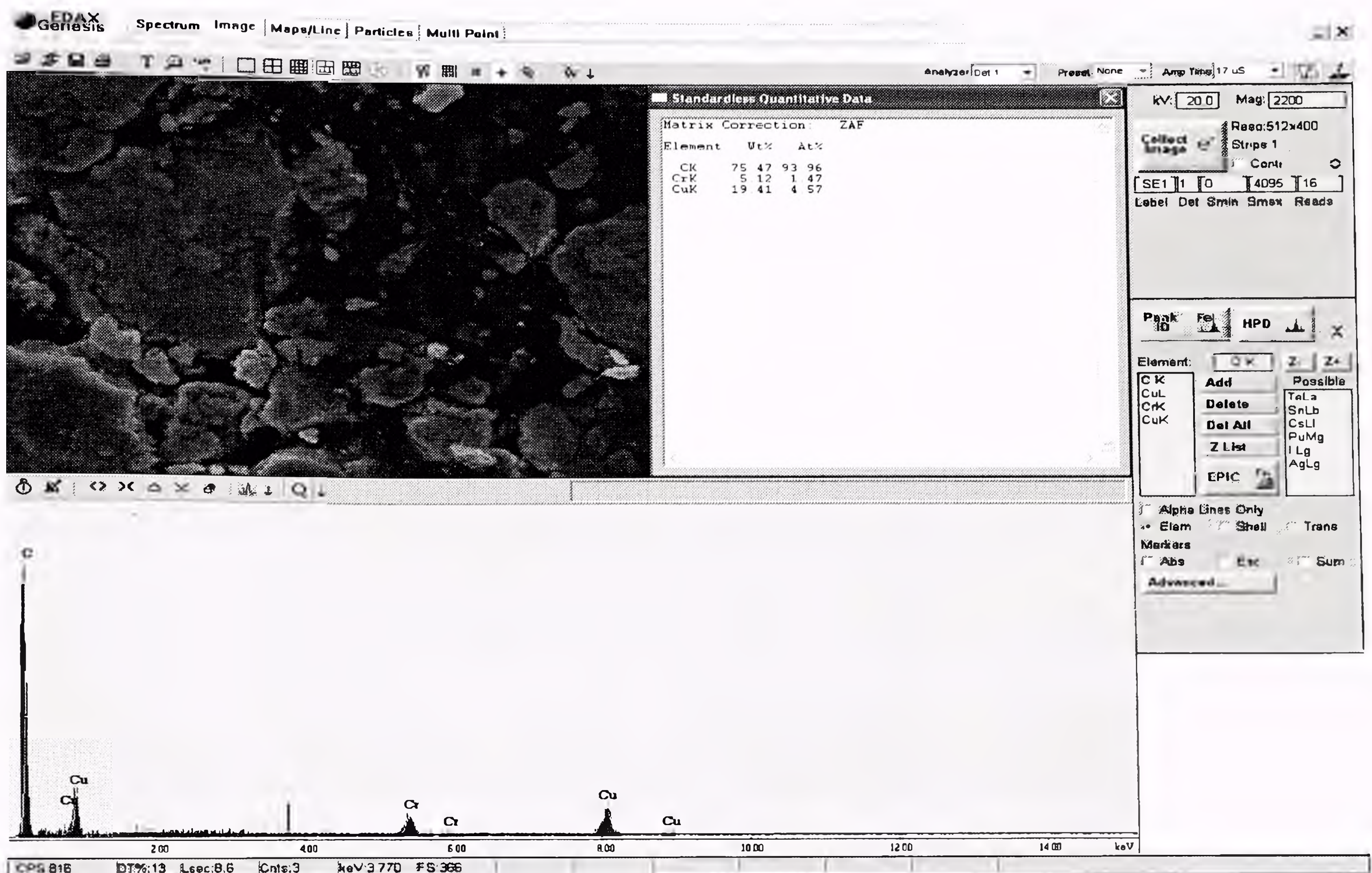

Figure C: EDS analysis of $\mathrm{Cu}-\mathrm{Cr} 12 \mathrm{Vol}$. \% MWCNT.



Figure D: BES of $\mathrm{Cu}-\mathrm{Cr} 14 \mathrm{Vol} . \% \mathrm{MWCNT}$. 


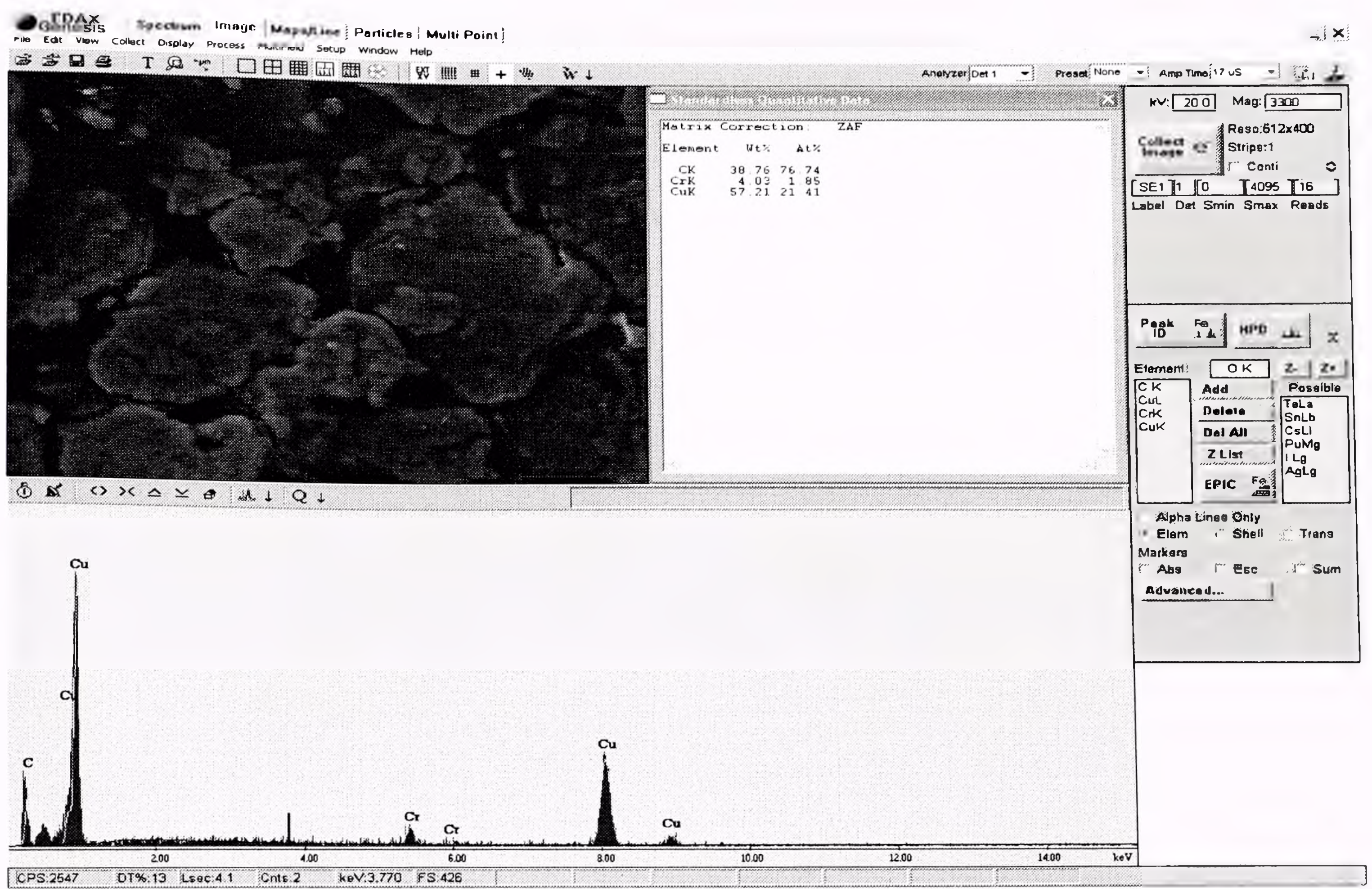

Figure E: EDS of Cu-Cr 14 Vol. \% MWCNT.

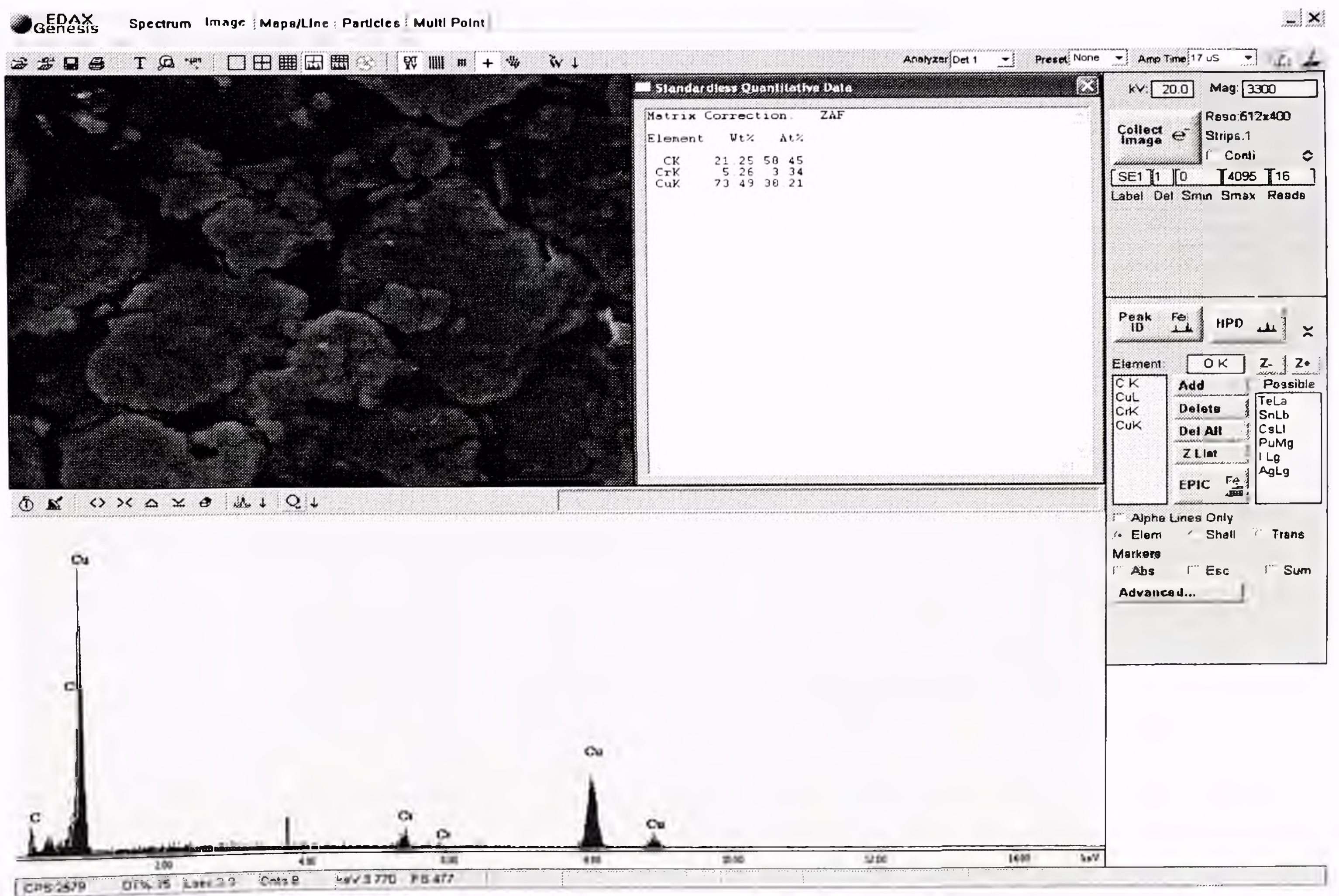

Figure F: EDS of $\mathrm{Cu}-\mathrm{Cr} 14 \mathrm{Vol}$. \% MWCNT. 


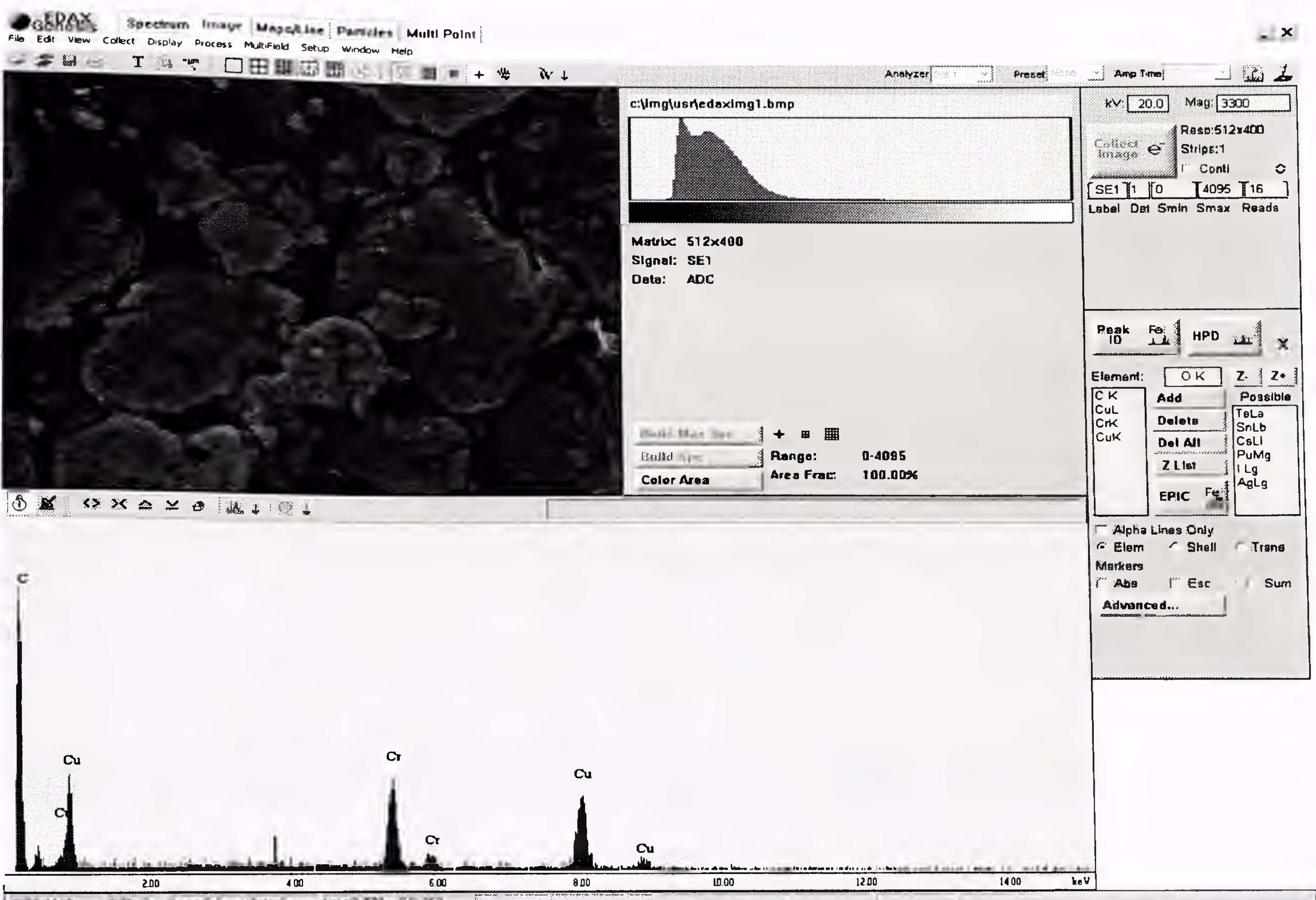

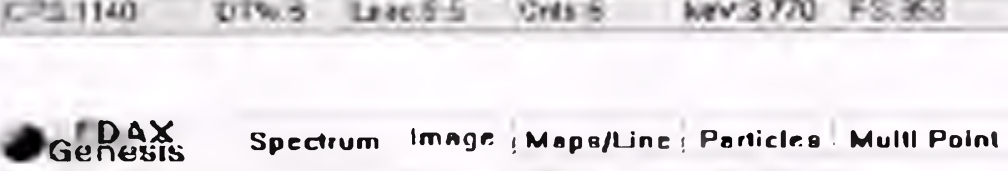

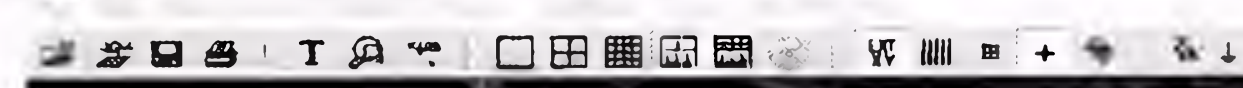

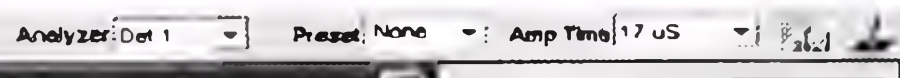

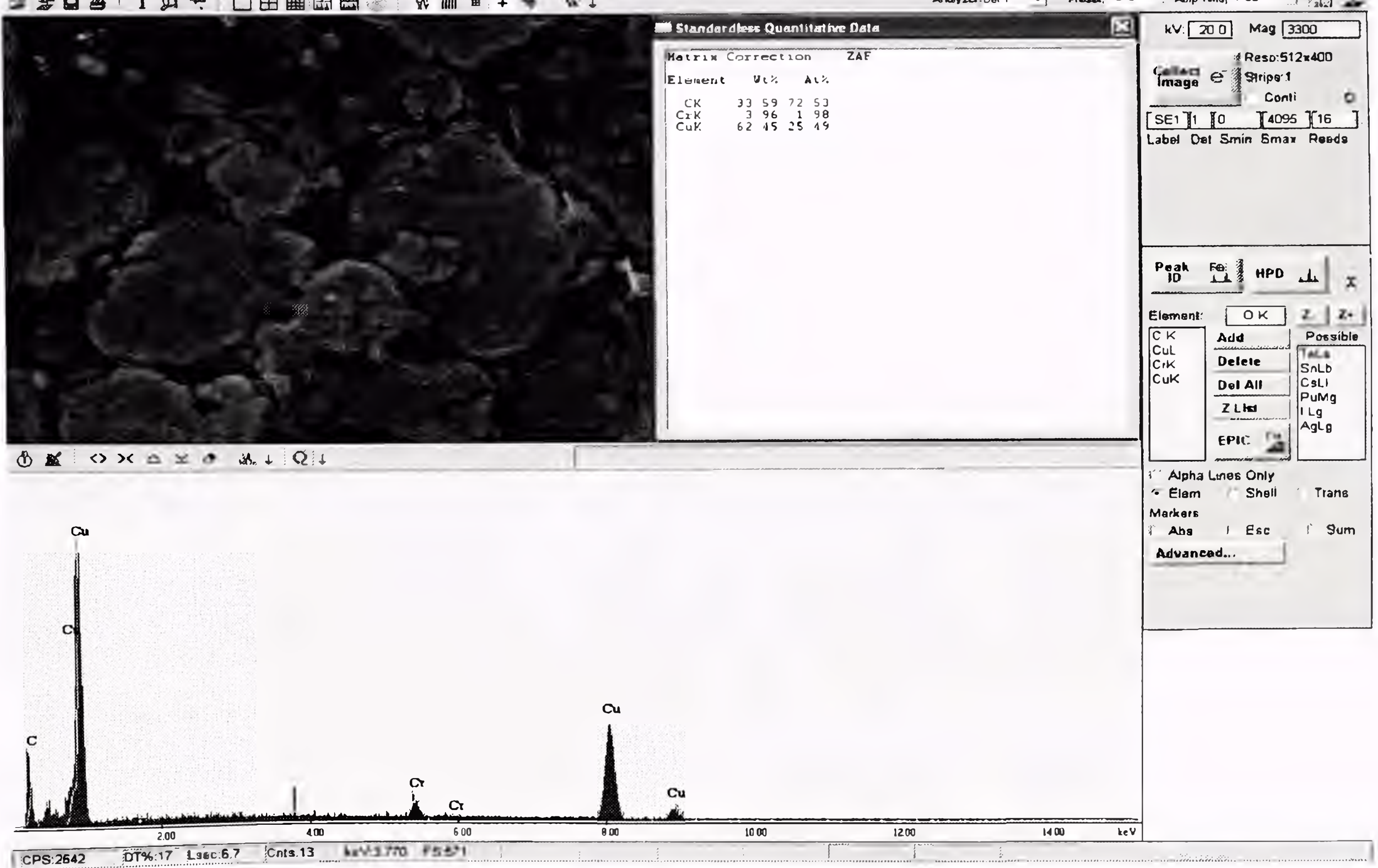

Figure G: EDS analysis of $\mathrm{Cu}-\mathrm{Cr} 16 \mathrm{Vol}$ \% MWCNT. 


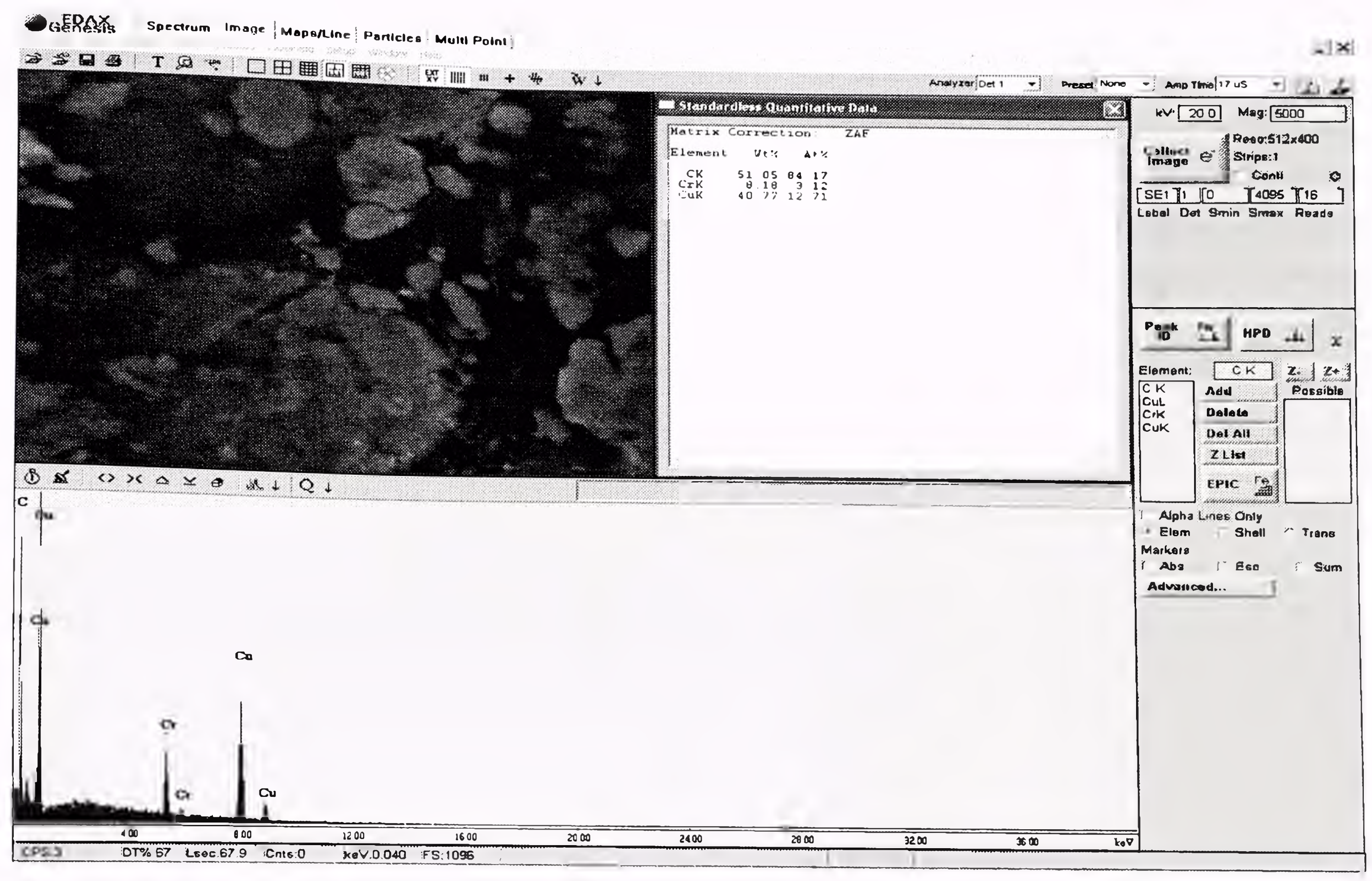

Figure H: EDS analysis of Cu-Cr 16 Vol. \% MWCNT.

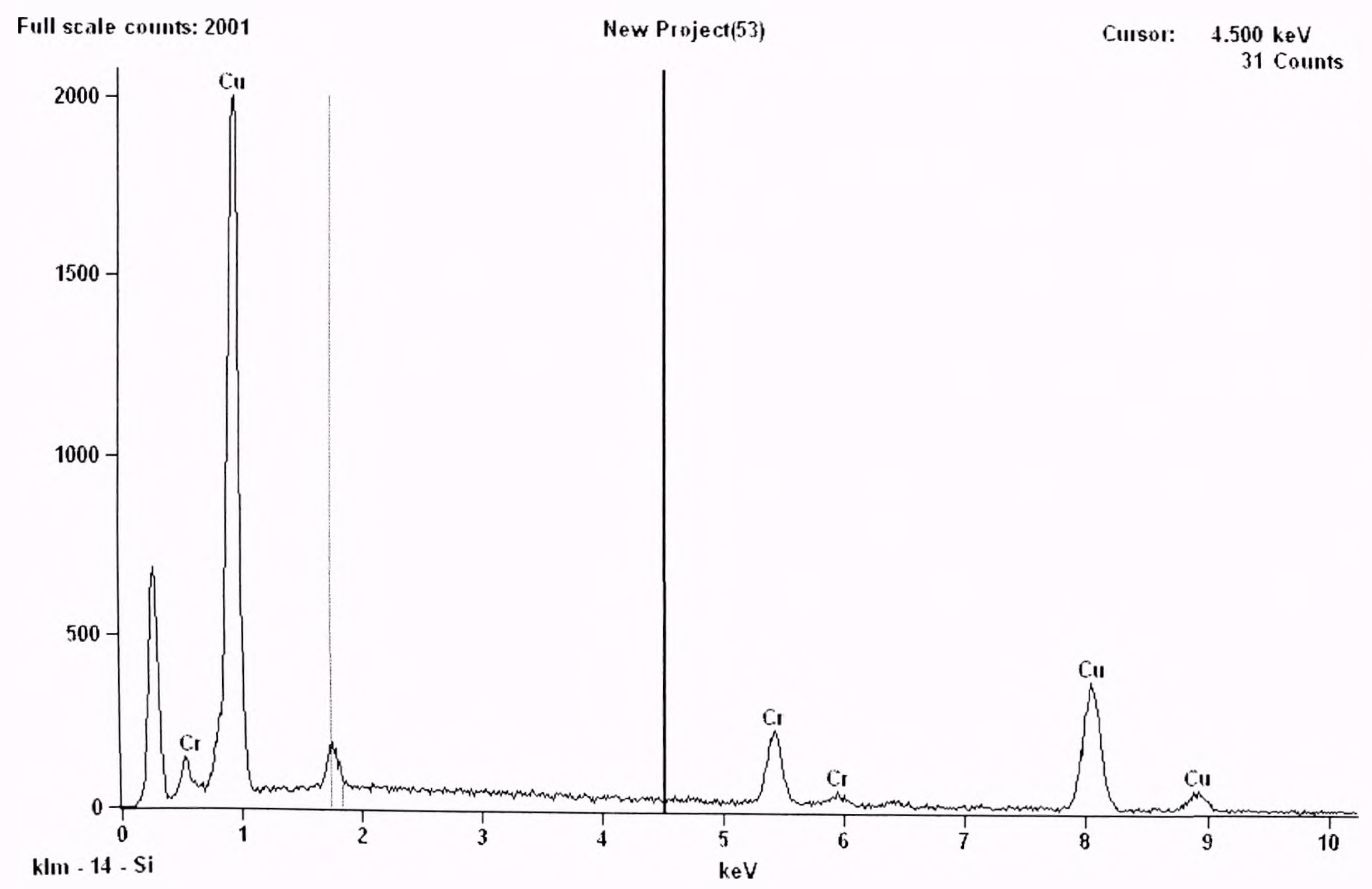




\begin{tabular}{|c|c|c|c|c|}
\hline Element Line & Weight \% & Atom \% & Formula & Compnd \% \\
\hline $\mathrm{CrK}$ & 13.40 & 15.90 & $\mathrm{Cr}$ & 13.40 \\
\hline CrL & $-\cdots$ & - & & - \\
\hline $\mathrm{CuK}$ & 86.60 & 84.10 & $\mathrm{Cu}$ & 86.60 \\
\hline$C u L$ & $--\cdot$ & - & & - \\
\hline Total & 100.00 & 100.00 & & 100.00 \\
\hline
\end{tabular}

Figure I: EDS analysis of $\mathrm{Cu}-\mathrm{Cr} 8 \mathrm{Vol} . \%$ MWCNTcompact sintered at $750{ }^{\circ} \mathrm{C}$. 